\title{
Virtual Media and Children's Everyday Play
}

Seth Giddings
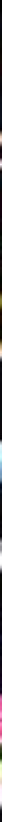

Gameworlds 



\title{
Gameworlds
}

\section{Virtual Media and Children's Everyday Play}

\author{
Seth Giddings
}

Bloomsbury Academic

An imprint of Bloomsbury Publishing Inc 


\title{
Bloomsbury Academic
}

An imprint of Bloomsbury Publishing Inc

\author{
1385 Broadway \\ New York \\ NY 10018 \\ USA
}
50 Bedford Square
London
WC1B 3DP
UK

\section{www.bloomsbury.com}

\section{BLOOMSBURY and the Diana logo are trademarks of Bloomsbury Publishing Plc}

First published 2014

Paperback edition first published 2016

(C) Seth Giddings, 2014

All rights reserved. No part of this publication may be reproduced or transmitted in any form or by any means, electronic or mechanical, including photocopying, recording, or any information storage or retrieval system, without prior permission in writing from the publishers.

No responsibility for loss caused to any individual or organization acting on or refraining from action as a result of the material in this publication can be accepted by Bloomsbury or the author.

\section{Library of Congress Cataloging-in-Publication Data}

Giddings, Seth

Gameworlds : virtual media and children's everyday play / Seth Giddings.

Pages $\mathrm{cm}$

ISBN 978-1-62356-632-6 (hardback)

1. Video games-Social aspects. 2. Virtual reality. 3. Play environments. I. Title.

GV1469.34.S52G53 2014

$794.8083-\mathrm{dc} 23$

2014009329

ISBN: HB: 978-1-6235-6632-6

PB: 978-1-5013-1829-0

ePub: 978-1-6235-6802-3

ePDF: 978-1-6235-6389-9

Typeset by Integra Software Services Pvt. Ltd.

Printed and bound in Great Britain 


\section{Contents}

Acknowledgements vi

List of Illustrations vii

Introduction $\quad 1$

$1 \quad$ Virtual and Actual Worlds 17

2 Virtual Media and Children's Everyday Play 35

3 Microethology: Methods for Studying Gameworlds 55

4 Media Worlds $\quad 67$

5 Soft Worlds: Play with Computers $\quad 89$

6 Play Grounds: The Material and Immaterial in Play 117

7 Real Worlds: Realities, Virtualities and the Protopolitics of Play 137

$\begin{array}{ll}\text { Bibliography } & 162\end{array}$

$\begin{array}{ll}\text { Index } & 175\end{array}$ 


\section{Acknowledgements}

This book is one material result of over a decade of interwoven academic research and family life. The gameplay event that is described throughout was recorded in 2002 when my children Jo and Alex were 4 and 3 years old. I was working on my $\mathrm{PhD}$ with Martin Lister, collaborating on a book on new media with him and our colleagues in the then School of Cultural Studies - Jon Dovey, Kieran Kelly and Iain Hamilton Grant. With Jon and Helen W. Kennedy, I set up the Play Research Group, which later welcomed Rune Klevjer, Hanna Wirman and Patrick Crogan. These projects, and friends, all shaped and encouraged my ideas and experiments. I have worked particularly closely with Helen - I thank her for friendship, generosity and support.

The Lego Racers microethology was published in Growing Up Online: Young People and Digital Technologies (2007), a book edited by the lovely Sandra Weber and Shanly Dixon. I would like to thank them both for their early encouragement of this work. Extracts from this chapter are reproduced by kind permission of Palgrave Macmillan.

Parts of Chapter 5, 'Soft Worlds', are taken from 'Events and collusions: A glossary for the microethnography of videogame play' published in Games and Culture, and included here by permission of SAGE. Thanks to Rebekah Willett for kindly sending me drafts of publications from the 'Beyond Text: Playground games and songs in the new media age' project.

Many thanks to the young people (and their parents) who have kindly allowed me to document and publish aspects of their childhood: Lily and Josie Wilshire, Nico Hart and Sam Primarolo.

And, of course, Penny, Alex and Jo. Penny features only briefly in the accounts of play here, but her love and care - and her prolific and phantasmagorical imagination - infuses these little events and the flow of life in which they form. I thank her for her patience with me as theorizing about play has removed me almost entirely from its practice. Jo and Alex have been and continue to be a source of delight, wonder and intense pride as well as data. I thank them deeply for allowing me to study them, and for their permission to publish my findings in this book. After all, as they pointed out with a shrug, no one they know will read it. 


\section{List of Illustrations}

Figure I.1 The outdoor adventure of Pokémon 1

Figure I.2 Mapping a gameworld 4

$\begin{array}{lll}\text { Figure I.3 Constructing a Zook } & 11\end{array}$

Figure 1.1 The transduction of Lego Racers 17

Figure 1.2 Virtual and actual krakens 24

Figure 1.3 Tidal wave 25

Figure 1.4 Death games in Sandy Bay 30

Figure 2.1 Ugly giants, electric eels and shark-human-cats 37

$\begin{array}{lll}\text { Figure } 4.1 \text { Drawing Pokémon } & 81\end{array}$

Figure 4.2 Meta-cards 86

Figure 6.1 Prelude: Talking Star Wars 119

$\begin{array}{lll}\text { Figure 6.2 Sticks, swords and sabres } & 121\end{array}$

$\begin{array}{lll}\text { Figure } 6.3 \text { Hammocks and bean pods } & 129\end{array}$

$\begin{array}{lll}\text { Figure 6.4 Darth Maul stickers } & 133\end{array}$

$\begin{array}{lll}\text { Figure 7.1 Lego riot } & 141\end{array}$

$\begin{array}{lll}\text { Figure } 7.2 & \text { Phantasmagoria } & 148\end{array}$ 



\section{Introduction}

A photograph from a family holiday. Two boys in a campsite, sitting close together, but with their attention totally absorbed by the screens of the Nintendo DS consoles in their hands. Framed by trees, they are huddled close to a metrehigh post and its electrical sockets, their consoles plugged in via cables, plugs and adaptors, batteries charging after days of travelling without electrical hookup. They are playing, no doubt, a Pokémon game, lost in worlds that synthesize pastoral exploration and adventure that simulate a community of human and monstrous playmates and adversaries. For those anxious about the current state of childhood, this might seem a dark irony - an idealized childhood of open fields, woods and collective, imaginative play mocked in vivid colours, and an impossible ecology of Viridian Forest and Five Isle Meadow (Figure I.1).

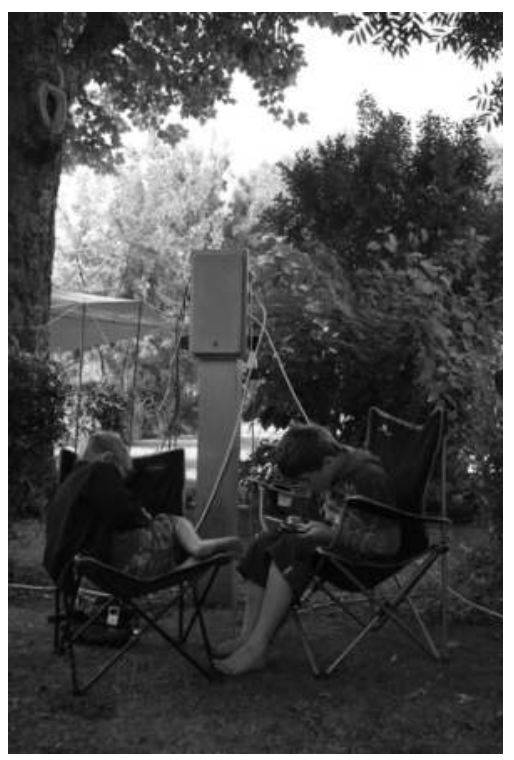

Figure I.1 The outdoor adventure of Pokémon 
I am reminded of a television natural history programme about barnacles living on thermal vents on the deep ocean floor. The hot, mineral-rich water builds up tower-like protuberances on which these molluscs survive - a few centimetres away from their tower they would perish in the deep cold. They are among the very few species that do not rely on the sun's energy for their life, as the other animals at these depths are sustained by debris drifting down from the sunlit waters way above. The children in the photograph are clinging to their devices and their life-giving cables and plugs, drawing imaginative sustenance from an environment utterly alien to the sunlight, trees, breeze and grass that surrounds them. From the tiny screen flows a bright, but sunless, world of exploration, adventure, play and puzzles.

But it reminds me too of photographs, taken by Fr Damian Webb in the 1950s and Shirley Baker in the mid 1960s, of young girls playing in the forbidding streets of Salford and other northern English cities. An environment ostensibly as inhospitable to play as the icy waters are to barnacles, filled with back-toback terraced houses opening straight onto the pavement. No grass, trees, no space other than pavement and cobbled street. ${ }^{1}$ These generations of girls, though, have transformed lamp posts into unlikely play equipment. Skipping ropes looped around the posts fashion a rudimentary swing. The mechanics of this improvized apparatus do not allow the free backwards and forwards motion of a conventional park swing, rather the girls swing out and round, in tight ellipses, brief flights and a vertiginous thrill. Like life itself, play will find a way.

Even in these two small examples there are numerous worlds in play. We cannot know, just from looking at the photograph, whether the girls' game is simply one of kinaesthetic exertion, or whether they have their own imaginative setting - such as magical flight - for their swinging. They may well have a rhyme or song, learnt from others via the oral folklore of childhood, or improvized in the moment. This may be a competition, a sport, the time of each leap and swing counted out and compared. The boys, though quiet and still, are playing - each in his own world, but perhaps comfortable in the presence of the other, perhaps aware of the sunlight and fresh air at some level. Meanwhile, the videogame software is generating its own dynamic microcosm: stories unfold, battles are fought and monsters collected as the boys click their way through the synthetic environment. The Pokémon universe extends beyond the console, across media platforms from comics to television, cards to toys and permeating everyday 
thoughts and conversations; this holiday was characterized by long journeys in which elaborate scenarios and dramas were spoken into existence. Pikachu, Charmander and others by the dozen evoked, summoned, intangible yet present between the children in the car.

\section{Space and time of play}

Children's games and imaginations in particular, but play in its broader and adult contexts too, are broadly understood in terms of space and time. Historians and anthropologists of play describe the ambiguous apartness of games in time and space: the marking out of the sports pitch or the microcosm of the game board, the marking out of time by the referee's whistle or the playtime interludes in the work or school day (Huizinga 1986, Caillois 1962). It is also fully part of everyday life, enacted by bodies and technologies, in physical environments and hemmed in by the constraints of more instrumental and practical activities. Playing takes place in the interior spaces and temporalities of the mind as well:

All play presupposes the temporary acceptance, if not of an illusion (indeed this last word means nothing less than beginning a game: in-lusio), then at least of a closed, conventional, and, in certain respects, imaginary universe. (Caillois 1962, 19)

Games and play are at once material and imaginary. They are real - but they trouble commonsense notions of reality; they offer multiple realities of fantasy, performance and intense experience. The 'gameworlds' of this book's title open up in fields, parks, streets, at home indoors, as well as in computer-generated space. The digital does not mark an absolute redirection of the history of children's play in space: play has always been characterized by some kind of virtuality. Moreover, even the modern virtual forms driven by sophisticated technology are not a clear break. Pre-digital electronic media such as television and video, and pre-electronic media such as cinema and literature have always been understood as worlds within worlds, thresholds to be crossed. Media worlds are playworlds, and these playworlds may appear in the gameworld as I describe it here, or follow their own orbits (Figure I.2). 


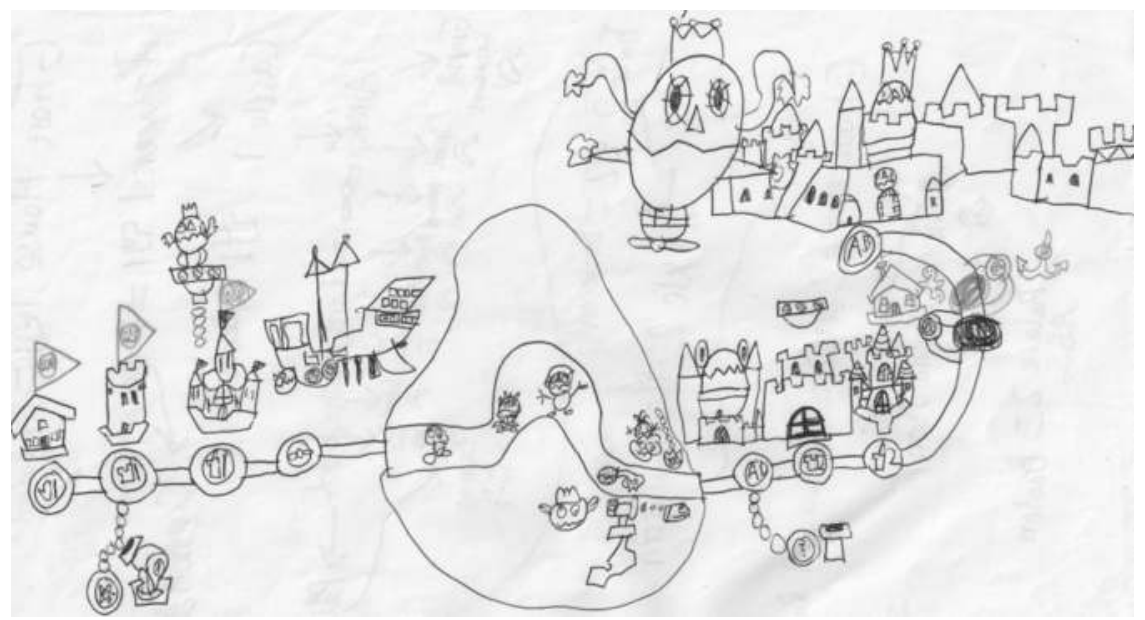

Figure I.2 Mapping a gameworld

\section{Media worlds}

All entertainment media, from books and comics to films and television, whilst not necessarily games, are playful. Viewers and readers cross a border into a fictional world and in so doing adopt a playful willingness to accept the characters, action and events as a kind of reality, and similarly to regard the evident artifice of genre conventions, narrative structure, spatial and temporal ellipses, etc., as real, or as real enough to warrant imaginative engagement and sustained attention:

The media have the capacity, indeed they entirely depend upon that capacity, to engage an audience within spaces and times that are distinguished - marked off - from the otherwise relentless confusions of everyday life. There is a threshold to be crossed each time we participate in the process of mediation. (Silverstone 1999, 61)

Theatre and comedy are among the popular media forms that most clearly acknowledge - and play with - their own compelling fantasies. As Shakespeare's Theseus puts it,

The poet's eye, in a fine frenzy rolling,

Doth glance from heaven to earth, from earth to heaven;

And as imagination bodies forth

The forms of things unknown, the poet's pen

Turns them into shapes, and gives to airy nothing

A local habitation and a name. ${ }^{2}$ 
The media spectator's imaginative and embodied engagement with the fantastical realities - the offending shadows - of play, film or sporting event is for Caillois one of the four main categories of play, mimicry or simulation:

Mimicry is incessant invention. The rule of the game is unique: it consists in the actor's fascinating the spectator, while avoiding an error that might lead the spectator to break the spell. The spectator must lend himself to the illusion without first challenging the décor, mask, or artifice which for a given time he is asked to believe in as more real than reality itself. (Caillois 1962, 23)

For Caillois, simulation is the way spectators are placed in relation to entertainment performances, in other words, the playful way they engage with the images and dramas before them. The term also encompasses children's imaginative play with both media and toys, and without. Any imaginative play entails role play or the meta-roleplay of the director of the drama, its staging, props and costumes. But sometimes the playworld is etheric, built on the spoken word, conversation or just thought, with no props, board or cards: airy nothing. Daydreams are perhaps the most ubiquitous moments of simulation, fleeting yet capable of briefly transforming the everyday:

Never shall I, Charlotte Brontë forget what a voice of wild \& wailing music now came thrillingly to my mind's-almost to my body's-ear nor how distinctly I, sitting in the school room at Roe Head, saw the Duke of Zamorna leaning against that obelisk with the mute marble Victory above him [...] I was quite gone. I had really utterly forgot where I was and all the gloom \& cheerlessness of my situation. I felt myself breathing quick and short as I beheld the Duke lifting up his sable crest, which undulated as the plume of a hearse waves to the wind [... ' Miss Brontë, what are you thinking about?', said a voice that dissipated all the charm, \& Miss Lister thrust her little rough black head into my face! 'Sic transit' \&c. (The Brontës [1835] 2010, 156-157)

Children's literature and other media have often been characterized by a 'worldness', shaped as much by the topographical impetus of a plot as by its temporality, prefaced with maps and with narratives following through landscapes, architectures, mythic structures and polities designed for the quest itself (e.g. J.R.R. Tolkein's Middle Earth, Tove Jansson's Moomin Valley or J.K. Rowling's Hogwarts and alternative London), through magical or nonsensical spaces that resist conventional cartography but sustain a powerful sense of place and other-worldness (Lewis Carroll's Wonderland and Looking Glass world, Norton Juster's Dictionopolis and Doldrums in The Phantom Tollbooth, Swift's Lilliput and Brobdingnag in Gulliver's Travels, the multiple dimensions of both 
Phillip Pullman's His Dark Materials and Enid Blyton's Far Away Tree) or more everyday environments and adventures (the fields and gardens of the small adventures of William Brown or Milly Molly Mandy, the lakes and islands of Swallows and Amazons and the challenging and varied environments of Michael Rosen's Bear Hunt).

Such worlds open up as virtual environments for play beyond the page, along with television, comic and film stories and characters, as resources for play in the playground, the park or the bedroom. They are to be lived in and played out beyond the act of reading or being read to - a stage and a dramatis personae ready for toys and dolls, Lego bricks and everyday objects, more or less faithfully replicated, hybridized with other storyworlds from media and literature or of the child's own construction. An example of this forms a central case study for this book, though it is a replication of the world of a computer game rather than a book or film.

It seems clear that the poeitic flow is not one-way as many writers, producers and game designers reflect on their own childhood world-building as a source for their adult work. Fragments in poems and diaries document Emily and Anne Brontës elaborate and sustained game/worlds of Gondal, Angria and Glass Town (the wellspring for the daydream mentioned earlier), whilst Satoshi Tajiri - the creator of Pokémon - cites his childhood fascination with collecting insects as his inspiration. As an adult, H.G. Wells revisited playful worlds by devising thorough rules for an intricate simulation of war with tin soldiers, set out in Little Wars.

Gondal and Angria is a classic example of what David Cohen and Stephen MacKeith call paracosms, 'spontaneously created imaginary private worlds, which for a considerable period recur in the child or young person, and therefore tend to become elaborate and systematised' (Cohen and MacKeith 1991, 109). These worlds - sometimes constructed from toys and blocks, sometimes as much talked into existence by friends or siblings or told as bedtime stories -are related to literary fantasy worlds, they suggest, but in play it is the world itself that fascinates, not its character or plot. A paracosm could be logical, governed by rules and constitutions, bedecked with flags and coats of arms and provided with a political history. Or, it could be infused with the spontaneous surrealism of children's play, where toys are animated and objects transformed. For example, the three cities of Petsland - the creation of a sevenyear-old girl and her ten-year-old brother - were organized according to the 
size of the toys that lived there. Petsville was home to larger toys, Juville for medium-sized and Hamville for the smallest.

Petsland and its activities provided an inexhaustible source of interest for the children. It lifted the restrictions of the 'real' world. 'We were "in charge" of Petsland and could manipulate its destiny. They had to care for twenty or thirty 'real entities (real to us, that is), that we could devote ourselves to'. (Cohen and MacKeith 1991, 25)

So, for all their marvelousness, these cities and lands had their own reality. On the one hand, the worlds and their denizens had a real presence within the children's lives, shaping their time, relationships and behaviour. On the other, events within them often had to correspond to a certain level of fantastic plausibility. For instance, the creators of Possumbul World, Dan and Peter, were monarchs, not gods:

They could perform no miracles: Plausibility was all. Dan recalls a well-meaning adult moving some toy to what you thought was a 'better' place without appreciating that such a change in location could not be effected by hand stretched out from heaven. Moving a toy would call for a crane, a lorry, and some personnel. Similarly the boys were embarrassed by the gift of a mounted toy soldier painted a uniform gold - until they hit upon the expedient of erecting it as a city statue! Realism was all. (Cohen and MacKeith 1991, 373-374)

The 'as if-ness' or 'sort of' reality of play is a theme throughout this book. Games and play generate their own versions of reality within the everyday, and players recognize (and play with) these rule-bound artifices. The rules of Bearland, for example, written up by two brothers along with its complex history and legends, scale maps and documents state (in 'paragraph 0:11') that 'there is no blaming things on the Bears, they are only alive to a certain extent' (Cohen and MacKeith 1991, 62).

Cohen and MacKeith's definition of paracosm is to some extent an artefact of both their research method and the nature of children's imaginative play itself. Asking adults to recall their childhood worlds will largely return the more sustained world-building events, those archived in maps and documents or simply persistent enough over time to lodge in the memory. Looking back from the viewpoint of adulthood the traces and products of play are visible, but the moments and activities of world construction - their being played into existence - are less accessible. These accounts of paracosms are memories, fixed 
and retold decades later. They may hint at the processes of play, but do not capture its dynamism, the deployment of toys and drawings in the generation of worlds in the distinct temporality of play. Like dreams, most play events are vivid and all-encompassing in their time, but immediately forgotten. Moreover, if children are preoccupied with play they are nearly always left to it by adults. School performances and competitive sport may be watched and photographed, but in their play with toys, words and worlds, children are generally left to it.

Most of the main examples in this book have something of the worldness of the paracosm but are more fleeting and ad hoc, without constitutions or written records but still compelling and engrossing for the players in the moments of their existence, transformative of everyday objects, media sources, bodies and environments. In fact, they may be repeatedly remade, sometimes in different contexts and with different media, and often media sources provide a connecting thread, stitched through successive playtimes, reminding, seeding and mutating.

\section{Gameworlds}

Play as an aspect of culture, popular media or mode of everyday lived experience is undertheorized in media studies, cultural studies in particular ${ }^{3}$ and the humanities and social sciences in general. It troubles previously reliable distinctions between, for instance, production and consumption, the everyday and the remarkable, the active and the passive, the obedient and the rebellious, the adult and the childish, leisure and work, even the human and the animal. The new interdisciplinary field of game studies has begun the task of seeking out and articulating thinkers and concepts that have taken play and games seriously, from Jan Huizinga's Homo Ludens in the late 1930s to more recent scholarship in disciplines from anthropology and psychology (Sutton-Smith 1997) to performance studies (Turner 1982).

Though influential theories of play, as we have seen, assert the apartness of play and games in time and space, this apartness is actually quite ambiguous. Whilst any particular imaginative children's game is intense in its fabulation and in the attention and concentration of its players, it can at any moment spin into a different game, or a player may leave, another be pulled in or it may fizzle out altogether, perhaps re-emerging minutes or days later. Video game play in particular is characterized by its immersive hold on the attention of rapt and nearmotioness players. And yet, the case studies and examples in this book describe 
a flow between these rapt and passional states and other playful activities. There are no obvious boundaries in time or space, but the players know when they are in the middle of a gameworld - there is a centripetal force that both draws in, but can then spin out, and ejects as well. (This dynamic is discussed further in Chapter 6, 'Playgrounds.) Gameworlds, virtual and actual, overlap and nest within one another, or butt up against others, allowing or inviting transition and transformation.

This book is not a comprehensive study of play then, but an articulation of notions and descriptions of 'free' imaginative or fantasy play, with the rulebound, mediating structures of digital media. Rather than classifying games, it pays attention to the transitions between games and transductions between the virtual and actual domains of play. This flow and these transductions are as characteristic and constitutive of play, as discrete rule sets, winning states or world boundaries.

Whilst all media engagement has a playful aspect, historically - as now children's media have also taken specifically ludic forms - the rule driven, abstract structures of board games and puzzles - as opposed to the narrative form of books, comics and moving images. Video games are a hybrid of playground and tabletop games, pinball machines and card games, along with the narrative or symbolic traces of books, films and comics. 'Gameworld' is a term common in the description of video games, particularly those games that involve exploration of, or orientation in, a simulated topography (adventure games, first-person shooters, platform games, 'sandbox' and simulation games).

What kind of gameworlds are built in the current era of digital media though? Video games at first glance collapse the media/literary world with the paracosm and other modes of imaginative play. The Harry Potter games, for example, not only provide, like the books and films, a fully realized environment with characters, histories, laws and rules, but they then furnish the player/reader with visually and audially immersive, navigable, virtual instantiation of that world. Play is directed, it seems, in this imaginary world by the logical and coded spaces, behaviours and algorithms of computer software (Figure I.2). A sense of horror at this mediatization - or even industrialization - of imaginative play permeates parental concerns and journalistic and academic responses to children's video game and digital media culture. The compelling, 'addictive' pull of video game worlds, it is feared, shifts play indoors and away from the physical and mental health giving activities of outdoor play. Play at computers and consoles 
is presented as solitary, anti-social, engineering a perverse intimacy with the synthetic and machinic at the expense of the natural and sociable realms of an ideal childhood.

The direst claims and predictions for the current and near future state of childhood are predicated on a misrecognition of the peculiar reworkings of reality that have always characterized play. Children's generation of imaginary and imaginative new worlds within the everyday is now valorized as natural, healthy and developmental, providing a pyschic foundation for adult life. It is worth noting that this view of play is a relatively recent historical one educators and moralists have also regarded play as something frivolous, wasteful and morally suspect to be tamed and channelled into edifying and educational activities (and echoes of this are evident in some contemporary work on play and education). But, by and large, imaginative, preferably outdoor and/or social, play is today seen as central to a healthy childhood. However, when uploaded or augmented through the artificial intelligences and virtual spaces of digital media, the 'healthy' play between fantasy and reality seems threatened. The photorealistic imagery and intense interaction not only outsource imagination, it is assumed, they so fully capture children's attention, for such long periods of time, that the 'more real than real' nature of play replaces any understanding of a world - natural or social - beyond the screen and controller (see Lister et al. 2009, 286-289).

Most of the dire claims made for children's game and Internet culture are founded on assumption and not on research. ${ }^{4} \mathrm{~A}$ key principle and motive force of this book is that we can only learn about play (about technology and culture) through observing and describing it. The meanings or effects of digital media forms such as video games cannot be read off from their images and scenarios, from the quality of their images and sounds, nor from assumptions about how they are played. This said, description and observation, whether scientific or anthropological, are not straightforward, unmotivated or unmediated activities (see Haraway 2004 or Latour 1987 on the former, and Clifford 1988 for the latter). The book is driven by descriptions and analyses of children's play with and around virtual media - and the creative and critical processes of description as an empirical approach. Chapter 3 ('Microethology') explains the concepts and methods I deploy in this ethnographic - or ethological research.

To talk of digital gameworlds in the same breath as the imaginary spaces of children's literature and the everyday fabrication of paracosms is to assert a strong continuity of this worldness through children's imaginative play and 
media culture and across centuries. It is to see video games - and play with and around video games - as a particularly vivid and relatively new instantiation of this continuity. However, as I will argue throughout this book, there are significant differences too. I want to complicate any straightforward culturalhistorical dialectic of continuity and change. Attention to children's literature and classic studies of everyday play help us to think about the newness of video games and virtual media, but the newness of video games and virtual media culture offers - I will argue - new ways of thinking about play and everyday reality before and after digital media.

\section{Sim you later}

The video recording captures a close-up of computer monitor screen. An alien arthropod, something like a short-bodied stick insect, but with four legs and a jointed tail, writhes in the centre of the screen, and at the centre of a nearly empty world consisting only of a chequered floor that stretches to a near horizon, and a small red bump that seems to be exerting a directional pull on the creature. The grey and white squares evoke both the rigid Euclidean geometry and visual aesthetic of early 3D computer-generated imagery, and the sequence in Alice Through the Looking Glass where the world is transformed into a giant chess board (Figure I.3). The monster's flailing limbs and bouncing gait inefficiently
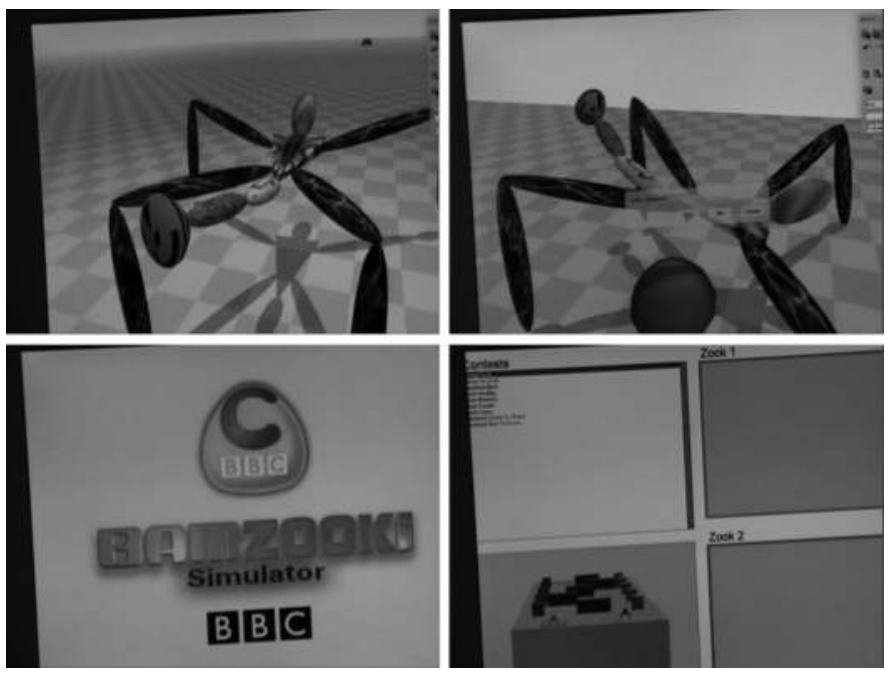

Figure I.3 Constructing a Zook 
but doggedly find traction with the virtual physics; it is - slowly - making its way through the abstract world. The video's audio track captures children's voices: two boys, one clearly at the keyboard of this computer application, the other - a younger, higher pitched voice - observing, commenting, on the creature's progress, and then riffing on the terminology of the application and other playful virtual worlds it brings to his mind.

The screen displays the name of the application, Bamzooki, along with the logo of the BBC. The software encourages children to construct sophisticated virtual animals from a set of malleable virtual shapes and forces. Once adept, children can work out which relationships of body parts, shapes, joints and behaviours can be assembled into an entity that can effectively race or fight those of other players (Figure I.3). These competitions could be conducted online, but also featured in a television series in which children could showcase and compete with their creations.

The older child has just exited from the 'Sumo' feature of the application. The name is apt as the ritual combat of these Zooks is won by one creature ousting the other from a small demarcated arena in combat characterized by momentum, timing and leverage rather than straightforward violence.

J: That makes him go faster doesn't it?

A: Yeah

J: It gives him more balance

A: Let's keep the Push!

J: See, he's going towards that red thing ... pretty fast!

J: Do you know what Follow means? That means we follow it... it follows ... we follow it ... it's going quite fast isn't it?

The Zook reaches the red marker

J: ... he just hops right over it. That's a good thing about it ... that it can hop over things

A: Look at its legs! Look how long its legs are

J: So it gives it a bit of an advantage ... if it's Sumo ... if it's Zook Sumo, doesn't it?

The boys seem to have collaborated on the design of their Zook and share their admiration for its capabilities. Push and Follow are key variables, determining the forward momentum of the Zook and the behaviour of the application's virtual camera, respectively. Jo has a moment of confusion over whether 'Follow' 
determines the virtual movement of the players as instantiated by the virtual camera ('we follow it') or whether it is the creature's instruction/instinct to follow the red marker.

\section{J: Let's quit, exit from this one}

The screen goes blank for a second, then a Windows desktop appears, cluttered with file icons and folders against a Pokémon wallpaper. The cursor clicks on an icon.

J: Right, Simulator ...

The screen goes blank again, then a loading screen appears, with the $\mathrm{CBBC}$ (Children's BBC) logo, the title Bamzooki in capitals with the subtitle 'Simulator'.

A: (yawns) What's this one called?

J: Bamzooki Simulator

Alex begins to play with the word:

A: I thought it said ... 'Bamzooki Sumo-lator'

J: (laughs) No (enunciating clearly and slowly) Sim-u-lat-or

A: See ... Sim ... (sing-song voice) Sim-U-Lator!

A: Hey, that's good, that's The Sims, when they say goodbye...

A: Sim You Later!

This tiny event, taking up around one minute and forty-five seconds of video, encapsulates many of the themes, objects and behaviours that will be explored in this book. Numerous worlds overlap, encircle or nest within each other. The Zook writhes through its 'micro-world' (Sudnow 2011 [1979]), immaterial yet real in its effects both within its synthetic environment and in the excitement and behaviours of the children. The boys have built it, and along with the software breathed sort-of life into it - it is, like the Bears, alive to a certain extent. It is certainly real, it is experienced, it affects the virtual and actual space and bodies within and without its own geometric landscape. The language and media form of simulation itself (here in its contemporary sense of computer modelling as well as in Caillois' categories) is adapted, translated and played with. It looks very different to the humanoid Sims but is for Alex of the same species. Like the Sims it is a kind of toy, but also like the Sims it is a kind of robot, its movement set in train by the players in their tweaking of the software variables, but conducted with a range of non-linear and autonomous behaviours that hint at an emergent 
sort-of life. Whilst we might study the game within the bounds of the screen, it loops out from the screen and through the eyes, nerves and fingers of the players, a cybernetic circuit of information feedback. These are media worlds too, designed and produced by media corporations, for non-profit edutainment (BBC) and for commercial ends (Maxis/The Sims), one sector of a sophisticated and global media culture for children.

They are games, for all their vivid imagery they are fundamentally abstract, structured by rules, motivated by ritual conflict (Bamzooki) or a more complex and open-ended set of goals and winning-states (The Sims, Pokémon). As media games, they operate or unfold less through the broadcasting or communication of messages from media producers - distant in time and space, and much more through the immediate and contingent instantiation of images, sounds and behaviours in intense events more akin to profane ritual or communion.

These worlds are nested within the everyday time and space of domestic life, and through the imaginative and personable cooperation of two siblings, but they also open up to each other ('Sim U Later!') and to other contiguous domains (the Pokémon universe). Later that day, Alex will draw a series of colourful monsters, a bestiary of his own design - a practice continuous with children's playful mark-making presumably since the origin of our species - but inspired by the artificial/natural forms, fictional and ludic attributes and algorithmic behaviours of Zooks and Pokémon.

The book is concerned with describing and conceptualizing play in the contemporary moment, a moment in which children's culture in the developed world is thoroughly suffused with the patterns and practices of digital media. It tracks the transduction of images and forms from the virtual gameworlds of video games across actual spaces of the home and playground, and their shaping of new games. The gameworlds in the central case studies discussed in this book cannot be separated into video game and actual play, rather they loop around and through both the virtual and actual domains. These gameworlds have a sense of their own universe but are not bounded by the edges of the virtual environment or TV screen. Obversely, the actual worlds of play with toys and in playgrounds, whilst ostensibly continuous with pre-digital games, are also unbounded - and must be understood as extensions of, and/or precursors to, play in computer and video game worlds.

This book, in part, explores the passage of long-established worlds of play and games into the digital age - the heterogeneous, yet completely continuous worlds of play: the body, toy worlds, physical environments and imaginative or psychic realities, out to the macrocosms of collective play, 
playgrounds and media environments and newer material and intangible spheres of virtual media and video games. As I will explain in Chapter 7 ('Real Worlds'), virtual media now suggest to us that play has always been something like virtual reality. ${ }^{5}$ Thus, a clear conceptual distinction between transformation and continuity can't capture the dynamic of contemporary children's technoculture. Rather, this book will attempt to rethink children's play and media cultures from the perspective of virtual media. I will begin with two sets of questions:

1. What is the nature of play today in an environment saturated with digital media devices, networks and applications? In what ways are longerestablished games and play patterns erased, sustained or transformed by newer computer-based media?

2. How might the virtual worlds and characters of the video game suggest new ways of thinking about earlier or longer-established patterns of play, for example, imaginary, literary and collective gameworlds with toys, books and outdoor spaces? Play has always inspired reflections on the membrane between fantasy and reality, imagination and the everyday, the material and the immaterial. In recent decades, technologies of virtual reality and the new communications and entertainment media of the Internet and the video game have posed similar questions. What is the relationship between the fantasmic images, characters and dramas of video game worlds - conjured from intangible flows of data and shaped by algorithms and feedback - and the vivid, immersive, often visceral, experiences they generate in players' bodies and minds?

A central concern throughout the book is to track play between and across the virtual and actual domains in everyday life, accepting Shanly Dixon and Sandra Weber's assertion that contemporary play is characterized by a 'flowing exploration through the permeable boundaries of the digital and the analog' (Dixon and Weber 2011, 487). The next chapter, 'Virtual and Actual Worlds', will begin this exploration.

\section{Notes}

1 There is, however, one characteristic of this forbidding environment that is more conducive to play than streets today: the absence of motor vehicles. 
2 A Midsummer Night's Dream, Act V, Scene 1.

3 There are a few notable and useful exceptions in media studies, for example, Fiske and Watts 1985, Silverstone 1999.

4 See, for example, Ben Goldacre on Professor Susan Greenfield's assertion that games and social media can be linked to autism and dementia (Goldacre 2011).

5 Or perhaps that ideas about play (and related biocultural phenomena such as dreams and language) have proved a resource in the construction of the technological imaginary of virtual reality itself, and its popular descendants the video game (Lister et al. 2009, 275). 


\section{Virtual and Actual Worlds}

The video captures a blurred, frenetic scene, children swooping through a small living room, with bright vehicles made of Lego bricks clutched in their hands. The soundtrack is a cacophony of voices making car noises and exhortations to 'come on!' and 'drive faster!' The two boys and cars, followed by the camera, trace a circuit or two of the room before arriving at a tall construction of larger plastic Mega Bloks. The race apparently over, the cars now trace a more leisurely route, up and over the Mega Bloks mountains and down to the beach and sea - expanses of yellow and blue crayoned onto sheets of A4 paper laid on the floor at the foot of the mountains. The video image is sharper now, the camera steady, and it closes in on the vehicles. Each is a fantastical machine, ornamental complexity at odds with the modernist geometries and colours of Lego, but still recognizable - and playable - as a car by sets of wheels and by a Lego minifigure ('man') as driver. They drive up and down over the mountains, along the coast, exploring the boundary between beach and sea (Figure 1.1).
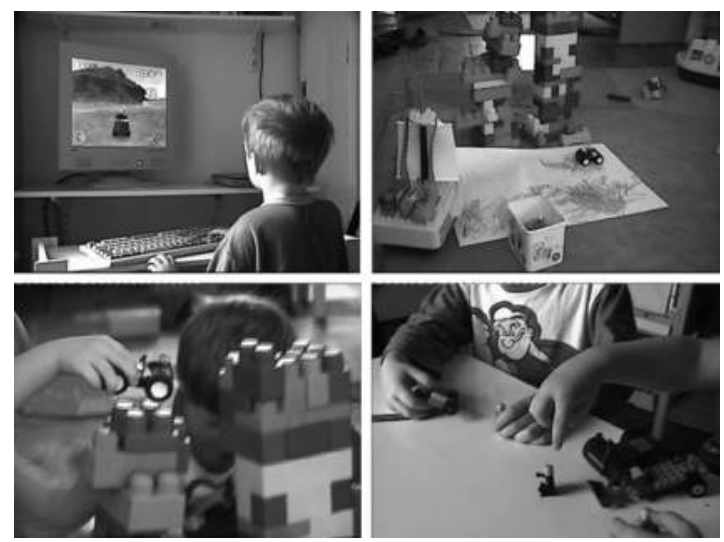

Figure 1.1 The transduction of Lego Racers 
At first glance, this home movie seems to document a familiar and unsurprising activity: small children in comfortable homes have played with commercially produced building blocks and toy cars for generations. And whilst audio-visual documentation of everyday play has only been possible with the availability of domestic video cameras, it is not hard to imagine similar dramas of engine noises, races and crashes echoing through living rooms and playgrounds for over a century. Yet there are distinctly new aspects to this playful event, aspects that prompted me to pick up the video camera and document it in the first place.

The fabrication of these plastic mountains and paper coastline was inspired by the playing of a computer game, Lego Racers 2 . The elder child had played this game avidly for a few days on a PC in the shared family space of the living room. The game's appeal was obvious - simulated Lego cars and drivers, familiar from his experience of playing with actual Lego were constructed on-screen from a set of options, then raced around an island circuit. At age four, the competitive racing element of the game was beyond him - he did not have the hand-eye coordination required to keep pace with the computer-driven racers - but the game offered instead a set of alternative playful possibilities from the construction of virtual cars to the engineering of accidents and crashes. In particular, and unlike most racing games, it allowed players to leave the race track and explore the virtual world beyond it. The Sandy Bay track loops around a fully explorable island with a beach, a Lego town and mountains with no marked distinction in virtual friction between track and other surfaces. ${ }^{1}$

So, whilst the computer-controlled competitor cars blithely completed their laps, the player and his car set off across 'Sandy Bay', a grassy island with steep hills surrounded by beaches, and dotted with a few Lego buildings and structures. With this realization, the game changed. It first shifted to a game of exploration. Jo adopted a free-roaming, exploratory approach, finding tunnels and tumbling down cliffs.

It was this expansive and explorable topography that captured the children's imaginations (the elder child joined by his younger brother for the Mega Bloks construction and drawing) and which was actualized: mountains, beach and sea, as a stage within which to perform the actions and sounds of motor racing.

My own interest, by now as much as a games researcher as a parent, was attracted by this 'translation' of the spatial dimensions of a virtual world into actual materials and experience. It hinted at a sophisticated engagement with 
aspects of popular media quite new to the form of the video game. Whilst, as discussed in the introduction, the narratives of children's media culture, from books and comics to films, cartoons and television, have often unfolded through the navigation of, or quest through, a fictional space, the virtual world of a video game demands a different kind of cognitive and imaginative engagement.

\section{Translations}

The actual race was performative, dramatic, in Caillois' terms simulational not agonistic, driven by a kinaesthetic enthusiasm for the dynamics and noise of speeding virtual cars, not by game rules or competitive structures. To best understand these translations, it is necessary to think through how virtual and actual playspaces and their players constitute each other, to describe something of the structure, operations and effects of the video game as virtual space and popular media software. The video game as hardware and software is an agent or set of agents in this event alongside the children.

Jo (whilst building Lego cars): We're going to Legoland, aren't we? And Disneyland...

The commercial strategy of cross-media licensing is well established in children's culture (Kinder 1991, Kline et al. 2003), and this game is not unique in making links between media forms and genres and toys; other successful recent examples include, of course, Pokémon and games based on the films of Harry Potter, Star Wars, and James Bond. The nesting of video game worlds, as moments of play and as media objects, within the commercial spheres of children's media culture will be addressed in Chapter 4 ('Media Worlds'). However, Lego Racers 2 also makes direct and witty links between the gameworld and its objects, and the physicality of Lego as a toy. For example, the player at the start of the game is given the option of building his or her own car and driver. A menu system offers ranges of virtual bricks from which figures and vehicles can be built. In the game proper, crashes result in bricks breaking off from the cars. Whilst the cars in the game are controlled in ways familiar from other racing games, they are represented explicitly as Lego cars, built from bricks and implicitly - acknowledging the pleasures of play that constitute the flip side to Lego's promotion of its toys as for construction - destructible back into bricks. 
[C]hildren's play flows easily on and off line, in and out of roles, weaving back and forth from the imaginative to the actual. It is in this blurring of boundaries between physical and cyberspaces, between the virtual and the actual that children create playspaces for themselves.... (Dixon and Weber 2011, 488-489)

Ethnographic research by Shanly Dixon and Sandra Weber suggests a number of ways that virtual and actual playworlds coexist and flow into one another. Rejecting nostalgic discourses that assume the loss of 'traditional' types of play in digital culture, they find instead strong continuities between longerestablished outdoor play and the domestic, indoor and virtual activities of video game play. The latter, they argue, share with the former creative and imaginative possibilities and are not separated off from each other: the boundary between digital and analogue is thoroughly permeable. A 'game' might flow between a backyard and a video game world, drawing on all manner of material and symbolic resources at hand and from popular culture. For example, they study the play preferences of two young friends in a game of Pikmin. The players explain the game's premise to the researcher thus:

Tucker: There's this guy from a planet and his name is Omar. Before he left on this journey he went to his wife who made him this soup with these carrots and when he left there was this asteroid who hit his ship and lands down on this toxic planet and he finds a carrot and it's red and it's a carrot. He has oxygen for thirty days and so he has thirty days to recover all the parts of his ship so that he can return back home and so every day you finish you get time to picnic so you bring him to these onions and they have feet and there are these flowers and the Pikmin will destroy the flower so you actually have thirty days...

Rowan (Impatiently): 'So we play now?'

Then, as they play the game, they too adopt an exploratory approach to the gameworld, not initially at least, following the actions that will facilitate the optimum progression through the game. The researchers link these exploratory, spatial pleasures directly to the boys' play outside, their dens and hideout in an alleyway ('an ostensibly classic childhood playspace') that runs behind their apartment block:

Shanly: 'What do you like about the game?'

Rowan: 'It's an adventure but there is action too.'

Tucker: 'We like to search for the parts of the ship.' 
The planet in the game Pikmin acts as a playspace for the boys when they are unable to play outside. Their interaction on the virtual planet appears to be similar to their play in their secret hideout. In fact, at times they imagine the secret place as a spaceship, perhaps inspired from their play in the videogame [...] Our interpretation is that both spaces serve to characterize the appeal of secret childhood spaces [... ]: they both feel concealed and secret; they are spaces where a child might slip off alone escaping from daily demands; and they are places in which to fantasize and dream. (Dixon and Weber 2011,486)

Children's imaginative play has a powerful gravitational pull, dragging all manner of physical objects and symbolic material into the orbit of any particular game or event of play. As well as the interconnected playspaces of the virtual and actual, videogame characters and actions join other media images, everyday and familiar scenarios and stories and songs from the oral folklore of childhood itself. All of these are gathered together, broken up, reassembled and synthesized in sustained collective performances, or in fleeting moments of chat, song or gesture. Andrew Burn describes the semiotic maelstrom of the primary school playtime:

Both boys and girls [...] make ambiguous or general references to elements of action, imaginary worlds, objects or weapons, and character types which might be associated with computer games. For boys, these include avatars, wizards, ninjas, pistols, bazookas, war, the SAS, and zombies; while for girls they include avatars, wizards, fighting guns, princesses, witches, fairies, monsters, robots, ghosts, superheroes and fairytale characters. [...] These represent hybrid influences which might include computer games, but might also include film, fairytale and folktale. (Burn 2013, 127)

At times, moments of play will crystallize as a direct instantiation of a video game world, with its specific interactive conventions or media-technological form. Burn gives an example of the inflection of a timeless play mechanic, hiding and chasing, with a very new one, the 'stealth mode',

one of the boys mentions looking down, for example, referring to the shadowed face of the cowled player-character in Assassin's Creed ' ... if you don't want to be seen in the playground you just sort of blend in ... stealth mode. Look up, then look down - like Assassin's Creed'. (Burn 2013, 133)

Girls' play in this particular study, Burn notes, tends not to be so closely based on specific named games or characters as that of boys, but it was still clearly shaped by videogame forms. Whereas the boys would re-enact the action of Call of Duty: Modern Warfare 2, complete with named characters ('Soap' MacTavish, 
for instance), girls conjured up more generic 'ghosts' within 'levels'. He gives an account from another game observed as part of the same research project. A group of five-year-old girls in a London playground were stepping in and out of plastic hoops lying on the ground. Two of the girls were interviewed in brief moments between the game sessions:

Rebekah: What are the rules of the game you're playing?

Lily: The rules are, the gaps in between, you have to go through them, and it's a maze where you have to go through. If you go in a hoop, then you're out for five minutes.

[...]

Sophia: But first we were allowed to step in the hoops, and now we're not.

Lily: And in Level Two, we have to go through a sort of maze, you see (points over wall at players).

Rebekah: Oh, there are different levels of the game!

Sophia: After you have to go in the gaps, then you have to go in the hoops, then gaps, then hoops again -

Rebekah: Oh, ok.

(Burn 2013, 137)

For Burn, these girls are 'constructing a ludic system by defining rules, and then adapting them. They are also aware of specific game structures such as the maze, a structure common to ancient forms of play and to certain genres of computer games' (Burn 2013, 138). ${ }^{2}$

\section{Mr Happy and the Age of Mythology}

A sunny afternoon some five years after the Lego Racers 2 game, the boys were playing around a paddling pool in our backyard. Rather than paddling in the water, they crouched beside it and played with toy boats and a large collection of Gogo's Crazy Bones, small collectable figures that were a craze at the time. ${ }^{3}$ My impetus for documenting their play this time was primarily aesthetic - the bright sunshine reflecting from the rippling water over the bright blue plastic of the pool, the vivid toys and soft skin of the children. The gameworld seemed as fluid as its watery medium. Much of it appeared to be delirious nonsense, a more or less inchoate succession of dramatic exclamations, snatches of improvized song, but some themes drifted through with the flotsam: pirates and vikings, 
an ambiguous character called 'Mr Happy' and numerous micro-dramas of drownings, hostage-taking and treachery. I left a digital voice recorder running to capture an hour or so of the sounds of play (see Giddings 2011). Studied later, the compiled audio track and photographic sequence identified some significant lines of imagery and action, including clear connections with the boys' recent video game play. A Playmobil pirate ship with its striped sail seems to have been the conduit for a summoning of Age of Mythology - a favourite PC game of Alex's at the time - connecting with both the Norse and the Greek worlds of the game and its software mechanics (game levels, mini-games, 'god powers'). As I edited the images and overlaid them with footage from the game itself, other visual and thematic resonances became obvious, from the intense blue of both the paddling pool and the digital sea to a striking realization that the crayfish, deployed as a giant monster to capsize the toy boats, looked - with its extensive antennae - and behaved - gigantic in its relative scale - remarkably like the krakens that Alex would gleefully unleash to wreck his virtual Greek ships (see Figure 1.2).

J: No, it's not like a computer game

J: Everybody's back to normal, everybody's back in their boats

A: Apart from the little boats have been totally destroyed

J: Those were mini-games, just mini-people...

J: They're just fun to kill

A: You and me don't try to kill each other, we just kill the little boats

J: Yep, with people on

A: Don't let them aboard you because they're really good at fighting

J: But some people survived, they're the shipwrecked guys ... it's a new ... like ... level, level-like thing...

A: Look at my ship! Raah! Raah!

J: Look, they're all dead

The play continues for ten minutes or so, with plenty of drownings and lifeboat rescues. Mr Happy makes his first appearance: he seems to be a multiple entity formed from two or three of the submerged Crazy Bones figures (each with an exaggerated grimace printed onto his plastic head) and a sing-song refrain:

A: Mr Happy ....

A: Happy, so happy

A: Even when he dies... 

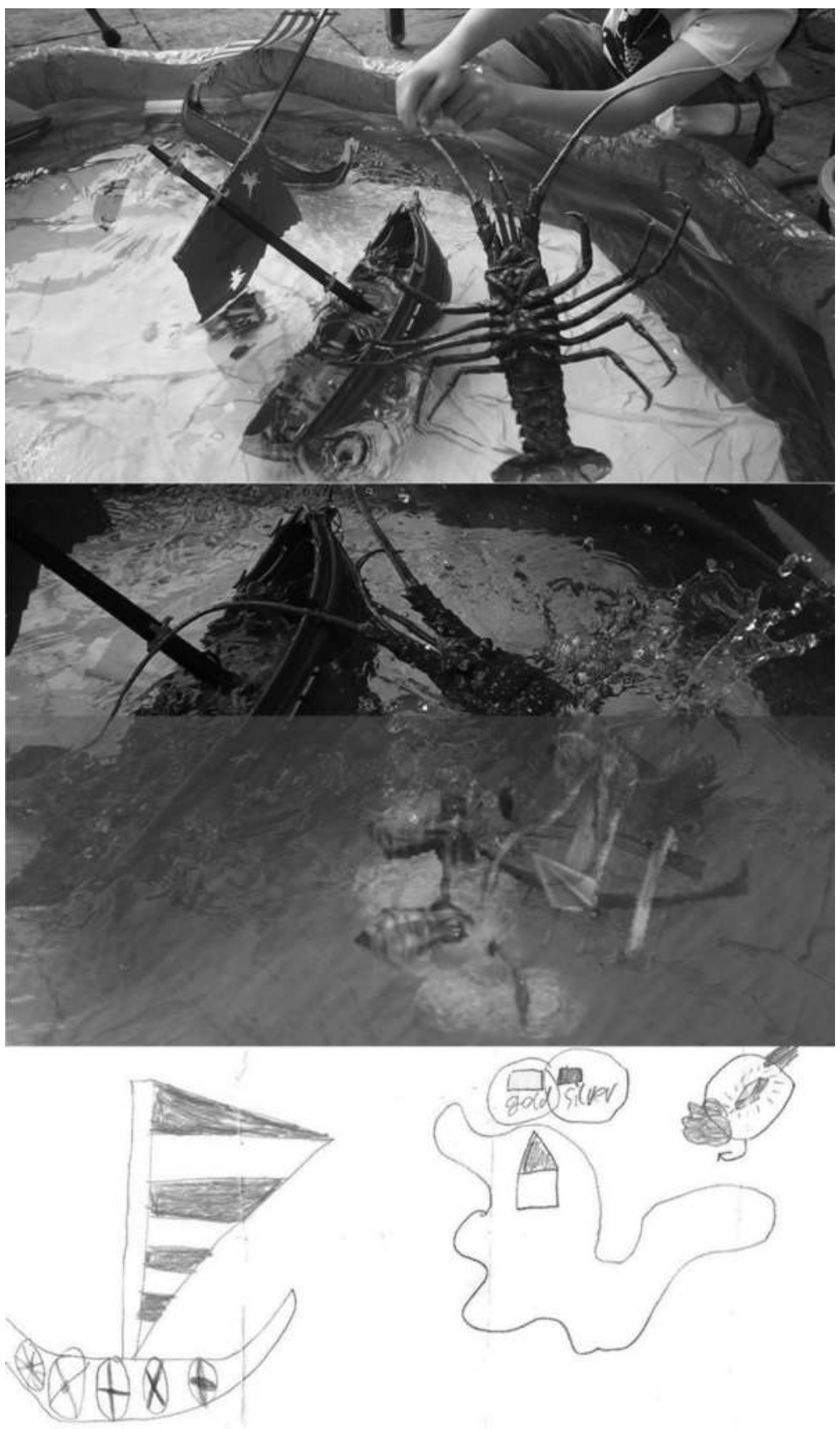

Figure 1.2 Virtual and actual krakens 
Later:

A: Look at Mr Happy! One of your guys survives so it's not Game Over. We've gone to the next level - everybody's on lifeboats!

Jo indicates a submerged Lego mini-figure, a black skeleton missing its skull:

J: We have sunk the traitor - apart from his head

J (traitor): 'I'll get you for this!'

J (traitor): 'Sorry, I didn't mean to be a traitor'

Jo grabs a large bucket, fills it with water and flings the water across the pool and the boats (Figure 1.3):

J: Tidal wave!

And again

J: Tidal wave!

$\mathrm{J}$ : This is the tidal wave

A: No, no, that's on bonus level

A: But guess what we do, we use god powers

A: Woo-argh Woo woo, we live! We live!

J: They just used a god power

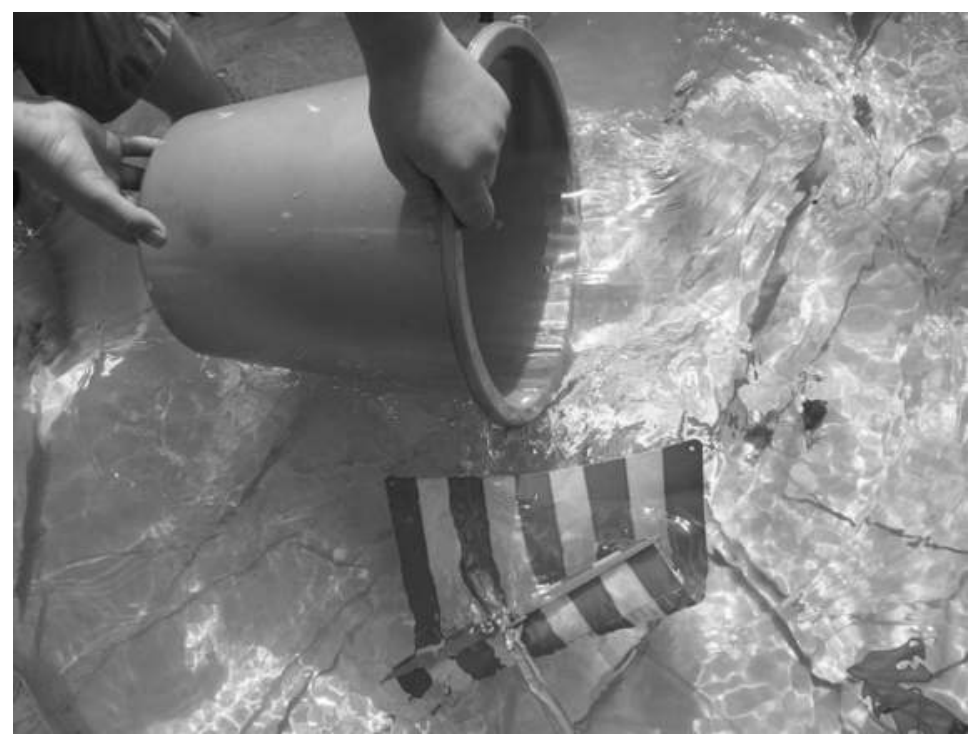

Figure 1.3 Tidal wave 
It isn't possible to transcribe the action and noise of the crayfish-kraken attack, but Figure 1.2 gives a sense of its momentary collision of virtual and actual worlds. There are varying homologies through which these virtual/ actual translations are inspired: water for water (both virtual and actual water a synthetically vivid blue), toy Viking longboat for virtual longboat and Greek galley, crayfish for kraken, bucket for divine power, to a plethora of characters and events that were spun into existence in these material-semiotic vortices, perfectly at home in this delirium of myth and disaster, but which seem to have no origin.

The peculiarly phantasmagorical character of much imaginative play will be described throughout this book, and addressed in detail in Chapter 7 ('Real Worlds').

\section{Back to Sandy Bay}

The players of the virtual gameworlds of Lego Racers 2, Pikmin, Assassin's Creed and video games in general loop out of them and back into their pre- or nondigital culture: to other media forms (books, TV, film) and to physical play (at home, in playgrounds, with or without toys or play objects), taking with them both narrative and formal elements.

As noted in the introduction, the improvized replication or performance of settings and environments from literature and media in children's play is hardly new. Spatial elements such as topographies, maps and architectures also shape games and play - from Lego and dolls houses to board games and theme parks. However, it was apparent that the Lego Racers reinvention was a response to the specificity of computer-generated gameworlds as simulations of space. The boys were not merely constructing a backdrop for a performance of computer game play, they were constructing it as space, and as an actualization of a dynamic virtual space with its own topography and simulated physics (friction, gravity, acceleration).

\section{Transduction}

But what is the nature of this flow of objects, images and forces across media and into everyday space? So far I have been calling it a 'translation', but this linguistic 
analogy does not capture the fundamental metamorphoses that must occur as an object - a Lego car, say - appears as at once a computer simulation, a screen animation and a physical object in a child's hand. Nor does it adequately account for the actualization of virtual/ludic forms and conventions such as game levels or virtual friction in play beyond the screen.

What is the nature of this movement across domains then? In educational psychology, the movement of ideas and images from media domains (literature, television) to children's behaviour is called transfer (Stevens et al. 2008). My use of translation was influenced by Bruno Latour's tracing of chains of agency through sociotechnical assemblages, the everyday operations and effects of power or agency as it is shifted or delegated from humans to a machine, or machines to machines (Latour 1992). Latour's conceptualization of these distributions of agency will be returned to later. The trans-prefix speaks of this crossing of domains, but neither 'transfer' nor 'translation' seem adequate to the transformation of entities as they perform these crossings. For all its semiotic and affectual connections, the virtual Lego car is a quite different entity from an actual Lego car. And an actual Lego car built as an instantiation of the video game car is quite different again. In its passage through the virtual world of the game and out again it has become a new entity - in the playful events described in this chapter it is a material-semiotic hybrid: no longer a toy animated by a child's imagination of the dynamism of driving, now a toy and child hybrid animated together in a simulation of the video game world. Whatever changes and transmissions are taking place they are non-linear and metamorphic. I have adopted instead the notion of transduction. Appropriately, it is used within cybernetics to denote

A process embodied in an input-output device for converting or coding without memory one type of signal, motion, wave or sequence of characters into another. E.g. a loudspeaker produces sound waves from oscillations, the human eye converts patterns of light into nerve impulses. Such devices are called transducers and are describable by a transformation or function. ${ }^{4}$

This sense of the transduction of information and patterns across machine and animal bodies or systems has been picked up by technocultural theorists and geographers to capture the contemporary movements, particularly of computer code: 'the constant making anew of a domain in reiterative and transformative practices' (Dodge and Kitchin 2005, 162). Adrian Mackenzie's development and application of the term resonates with the processes and phenomena I am concerned with here. Transduction tracks processes 
that come into being at the intersection of diverse realities. These diverse realities include corporeal, geographical, economic, conceptual, biopolitical, geopolitical and affective dimensions. They entail a knotting together of commodities, signs, diagrams, stories, practices, concepts, human and nonhuman bodies, images and places. They entail new capacities, relations and practices whose advent is not always easy to recognize.

A transductive approach promises a more nuanced grasp of how living and non-living processes differentiate and develop [...] To think transductively is to mediate between different orders, to place heterogeneous realities in contact, and to become something different. (Mackenzie 2002, 18)

Again we see resonances between concepts of worlds, dimensions and newness, arising from digital technologies and media on the one hand and play on the other.

I will return to these theories of the flow and transformation of agency in Chapter 3 ('Microethology'). For now, I will focus on their operation through the playful circuits of computer games - by simulated space and physical forces in particular. The vertiginous manoeuvres up and down the mountains generally had little effect on the car/avatar, beyond a few bricks shed on particularly spectacular impacts. However, it soon became apparent that the car/avatar could be destroyed if driven or dropped into the sea. Usually 'death' in a video game marks failure - temporary or terminal - and results in frustration for the player. However, given that Jo's exploration had no set goal and the fact that the game was configured to allow infinite numbers of lives, this repeated death became a simple game in itself, one in which he delighted. Jo would begin with the car/ avatar at the race start, then deliberately veer it off the track to pursue the shortest route into the sea and hence into momentary death. The car/avatar would then reappear instantly, in a swirl of stars reminiscent of graphic conventions from comics and cartoons of minor head injury.

A new variation emerged. Jo found that driving the car slowly and carefully into the sea allowed a more nuanced experience of drowning than that offered by plunging off a cliff. The car could be directed into the water and gently nudged deeper, until, just before its uppermost point (usually the top of the driver's head) was submerged, it 'drowned'. The motive of this new game, then, was the identification of, and the edging around, the precise point at which the game switched between life and death. The cars lurched up the cliffs, teetered at a vertex where dwindling virtual momentum succumbs to the faint but insistent pull of virtual gravity, and fell back, slowly, bouncing down to the beach. Down 
here the drowning game was re-enacted, the car and driver held over the drawn sea, hovering, descending, then emerging again. The video game world's liminal state between land/life and sea/death was, therefore, replayed out in an actual game environment, where the point of death (like the momentum/ gravity vertex) was determined by an embodied articulation of video game kinaesthetics.

On watching the video footage of Jo playing (often attended and encouraged by his younger brother), it became clear that these improvized games were constituted by the complex interactions among the gameworld's physics; the affordances of software elements (notably those of the car/avatar); the transmedial suggestions and humour of this particular game (linking it to prior knowledge of the Lego franchise in its actual instantiations); and the characteristics of more traditional children's play with toys, notably the pleasures of exploration and creative destruction. For example, one of the game's funniest features is that if a car suffers a particularly powerful collision then virtual bricks will fall off it. Enough collisions and the car will eventually be stripped down to a chassis (with no apparent effect on its capabilities as a vehicle). One more crash, however, and the player is left with the 'man' on his own to steer around the gameworld running delightfully on stiff little legs. We were all disappointed to find out, after much effort and experimentation with collisions and suicidal leaps into the path of non-player cars, that the game does not allow the man, as Jo put it, to further 'break into two legs'.

This feature offers visual pleasures (the familiar form of knee-less Lego legs are now more hilarious in their frantic animation) but it also highlights the operations of vehicular affordance in the game. It is an unusual and perhaps unique device. Games such as the Tomb Raider and Grand Theft Auto series periodically encourage or require players to guide humanoid avatars into vehicles, shifting control from the manipulation of human-shaped capabilities to motor-vehicle-shaped capabilities. In these other games, there are instrumental reasons for this (shape) shifting between different kinds of control/movement, and specific affordances are granted or denied as part of the design of the gameplay.

The transduction of the video game world and its conventions into off-screen play was varied and extensive. Alex, for example, adapted characteristics from the video game world into his own well-established micro-worlds of swimming creatures and dramas in the bath and bathroom sink. In particular, he developed the dramatic possibilities of immersion: he had found another way to 'drown' 
the car driver, fusing his ongoing fascination with toys and water with the video game-suggested event-horizon of life/death (Figure 1.4).

Not only were the images and dramas of the computer game being played out with real toys, but the physical and kinaesthetic ways the boys played with their actual Lego blocks were now quite different. They were not only continuing the game of racing Lego cars begun on the computer screen (its characters, scenarios and dramas), but were also playing with actual Lego as if it were a video game. They were, on one level, playing at playing a video game. For instance, the actual Lego cars and drivers were constructed through an enactment of the video game's menu-driven start-up processes: invited by the boys, not least because of the attention I was giving them, to join in their play (or rather, take direction from them), I was instructed to choose the cars and figures to be played with from a range they had built. Initially this choice was made as they were creating

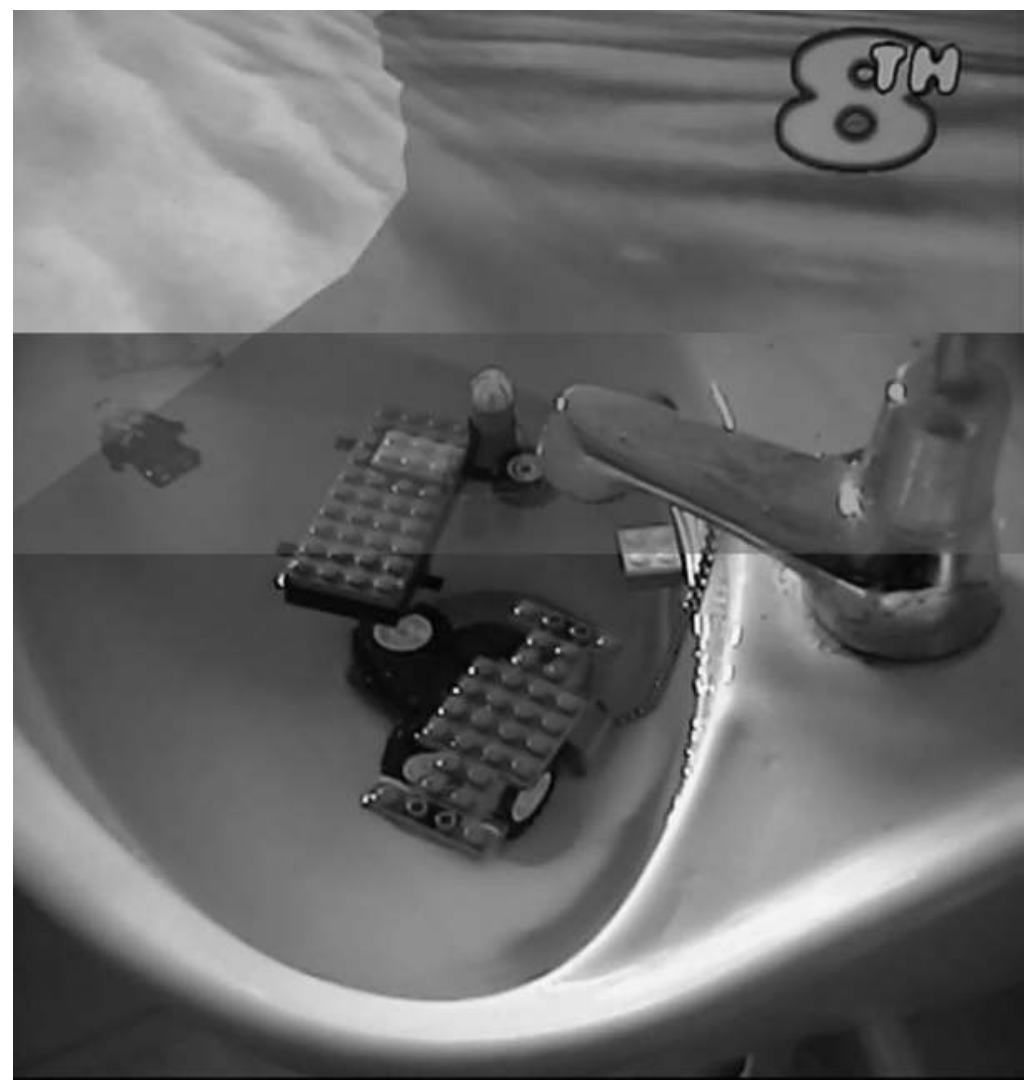

Figure 1.4 Death games in Sandy Bay 
the vehicles, but later the process was repeated more formally, with cars and drivers neatly laid out on the table. The process of selection was incorporated into well-established patterns of their off-screen play: on-screen, no qualitative or quantitative value is placed on any selection, whilst in our living room the boys offered me a choice of two menus, each comprised of vehicles they had built individually. A familiar sibling-parent politics of attention-seeking and turn-taking was brought to bear: the eldest boy was most insistent, so his car was chosen first; his younger brother was keen to keep his menu in play, though, asking, 'later, can you choose this one?'

Initially I was able to observe these transductions of agency and software structures because of the way I was positioned by the boys in relation to their actual game: I was not offered the chance to give any input into the design or construction of the actual cars (even for the one decision I was allowed - whether one driver should wear his baseball cap forward or backward - it was made clear to me that 'backward' was a preferable choice). Clearly I was not the empowered agent, or so it seemed. However, from studying the video record it appears that my role in these games did prove instrumental to opening a whole complex of actions, choices and translations arising from the peculiarities of the videogame medium and its articulations or channelling of agencies. It became clear that an entirely unexpected set of shifting identifications and transformations were at play in these shifts from screen to living room and back.

As the boys careened across the floor, racing each other, crashing into and leaping over the mountains, I was instructed to 'use the keyboard'. I was expected to play the role of the 'player', 'clicking' (with my fingers on the arm of a chair or on a book) imaginary keys to make the Lego Racers go 'forward!' and 'faster and faster!'. Of course my role was in some senses redundant - my finger movements had no influence on the direction or velocity of the Lego Racers (human or plastic) - yet the passion with which I was remonstrated when I stopped my performance indicated that this participation was, in some way, significant to them.

My role, then, was an apparently contradictory passive performance of interactivity. The videogame dyad, the circuit between player and avatar, was simultaneously collapsed and expanded: the boys and their cars were the avatars 'in the game', the agency of the player assimilated into the new boy/car/ avatar and the residue (the empty performance of key pressing and looking on) displaced to another, 'interpassive' body. The sheer complexity of these circuits, translations and feedback loops was brought home to me with the following 
sequence of events: at the start of the actual game, once cars and drivers had been selected, Jo asked me to write my name ('D.A.D.D.Y.') on a piece of paper, a translation of the familiar entering of the player's name or nickname at the start of the video game.

This activity was consistent with my passive player role, but when I asked Jo why he wasn't writing his own name, he replied, hesitantly, 'I don't have to - I'm the one who makes the Lego Racers go' I tried to draw him on this, excited at the implications of this for theories of identification in video games. That is, Jo might be identifying himself (through role play) directly with the computer or computer game rather than the characters, the Lego car or man. He was not the player and so he didn't 'sign in' but neither was he simply the 'avatar'. It is, then, the game itself, perhaps thought of as the game software or engine, that actually 'makes the Lego Racers go'. I asked Jo if he was the game, and he hesitantly answered 'yes'. I realized, though, that I was pushing him and that if he was 'playing as the game' in the sense I suspected, he wasn't aware of it or reflecting on it - at least not in the terms I was using. I stopped asking questions in this way.

The relationship, then, between virtual bodies and worlds and (playing) physical bodies and worlds is far from simple. Both are a kind of virtual world; both have their restrictions and affordances; both can be imaginatively inhabited by the children, animated by their actions (whether physical or console-mediated); both are capable of loose, improvisatory, paideic play or tight, rule-governed ludic play (Caillois 2001). (Burn 2013, 142)

Andrew Burn's theorization of the interpenetration of virtual and actual gameworlds supports my understanding of the Lego Racers worlds described earlier. I would suggest, though, that we are seeing also a set of more complex relationships or circuits. There are continuities in play and games here that resist claims that play with virtual media spells the end of 'traditional' forms of play, but there are also significantly new play environments and playmates emerging in virtual worlds, actual worlds and the transductive spaces between them. The challenge, therefore, is not to separate out the continuities and ruptures in the cultural history of children's play; it is to question how play can be rethought in the light of popular digital technoculture. Play manages to loop through and across these ostensibly incompatible domains. We need to look, then, at the connections, the flows, the circuits: what is transmitted, what is transformed, what is lost and what is made? 


\section{Notes}

1 This simulation of friction is effected through the game program slowing any vehicle that leaves the track for the ground surface that borders it - usually grass. It is a common device in racing games for guiding players on the game's preferred trajectory and rewarding accurate steering and cornering.

2 Even the most minute everyday activity can become charged with the ludic possibilities of videogame structures. I remember trying to get Jo ready as we were running late for school - he was around five years old. Sitting on the stairs, he waved his foot around in the air as I tried to get a sock onto it: 'Look! You're doing it on hard level!'

3 Though in their bright colours and differentiated forms (for collecting), Crazy Bones are very much a product of contemporary children's culture, in their name, shape and game mechanic (like Pokémon cards they function as both collectable object and game element) they are actually directly related to some of the most archaic play objects: the knuckle bones used for gambling since Antiquity.

4 Web Dictionary of Cybernetics and Systems: http://pespmc1.vub.ac.be/ASC/ TRANSDUCTIO.html. 



\section{Virtual Media and Children's Everyday Play}

\section{Events}

The subtitle of this book is 'Virtual Media and Children's Everyday Play'. None of these words has a simple, single definition, and they could be swapped around and still do some descriptive work for the book's themes: for example, 'children's everyday media and virtual play', or even 'everyday media play and virtual children'. The unstable core of my enquiry is not 'children', 'video games' or 'play'. For example, the Lego Racers account (see Chapter 1) cannot be reduced to a study of a particular computer game, to an example of a child's use of popular media or to an enquiry into the impacts of digital technology on everyday life. It cannot be disaggregated into a media cultural practice, a subject position, a set of technologies or even any straightforward relationship between the three.

The object of the descriptive studies that form the case studies in this book is not 'children' or 'video games', then, but the playful events in which these various entities come together. To talk of events is to foreground the temporal unfolding of video game play on- and off-screen, as well as the spatial dimensions of its overlapping gameworlds, and to establish the dynamic between the elements in play: a sense of entities coming together, material chains of cause and effect or feedback. Dan Fleming's consideration of the effects of diverse contexts for (in this case) toys in play is relevant here:

... the effects we are going to be interested in are simultaneously in the formation of an object and in that object's consequences within the processes that formed it. In a way, therefore, it might be better to talk about 'events' rather than 'objects'. (Fleming 1996, 10-11)

This chapter will first set out the key terms in the book's subtitle, questioning their conceptual and descriptive stability, before sketching some broader research 
contexts and influences, then finally explaining the conceptual and practical characteristics of the empirical approaches I have developed in this research.

\section{Anti-glossary: Virtual; media; children; the every day; play}

Glossaries offer concise, coherent definitions of key terms and concepts. As I have indicated, the key terms of this book's subtitle are unstable, ambiguous and multifaceted. I will use another small event of children, play and digital media to open up these terms to suggest how they might be deployed and studied.

The twins were bored. Their father had called round on their way home and was now engrossed in conversation with my wife, Penny. To keep the girls occupied we showed them the Photo Booth application on my laptop. The application uses the laptop's built-in webcam to capture still images and video and offers a wide range of filters, image manipulation settings and animated backgrounds for users to play with their own image. The girls, Lily and Josie, first filmed themselves dancing in a multi-screen posterized set of images suggestive of an Andy Warhol painting. Demonstrating the ability of some sets of twins to pick up on or finish each other's sentences, they quickly and spontaneously slipped into a brief synchronized song and dance:

L: I'm a good dancer, dancer, dancer, I'm a good dancer, dancer, dancer

$\mathrm{L}$ and J: I'm a good dancer, dancer, dancer...

L: And I'm strong, strong, strong

$\mathrm{J}$ : And I'm the main character, character, character

L: No you're not, I am!

This was followed by a series of video self-portraits that grew steadily more monstrous, first through the time-honoured game of face-pulling for the camera and then, using the distorting filters provided by the software itself, pinching, elongating and swirling their faces into delightful disfigurements - an augmented gurning (Figure 2.1):

L: Oi look really ugloi don't oi?

J: Yes, you dooo.

J: Do your face like this (she pushes up close to the camera) and you look like a giant!

L: Oooaargh ... are you a giant? 

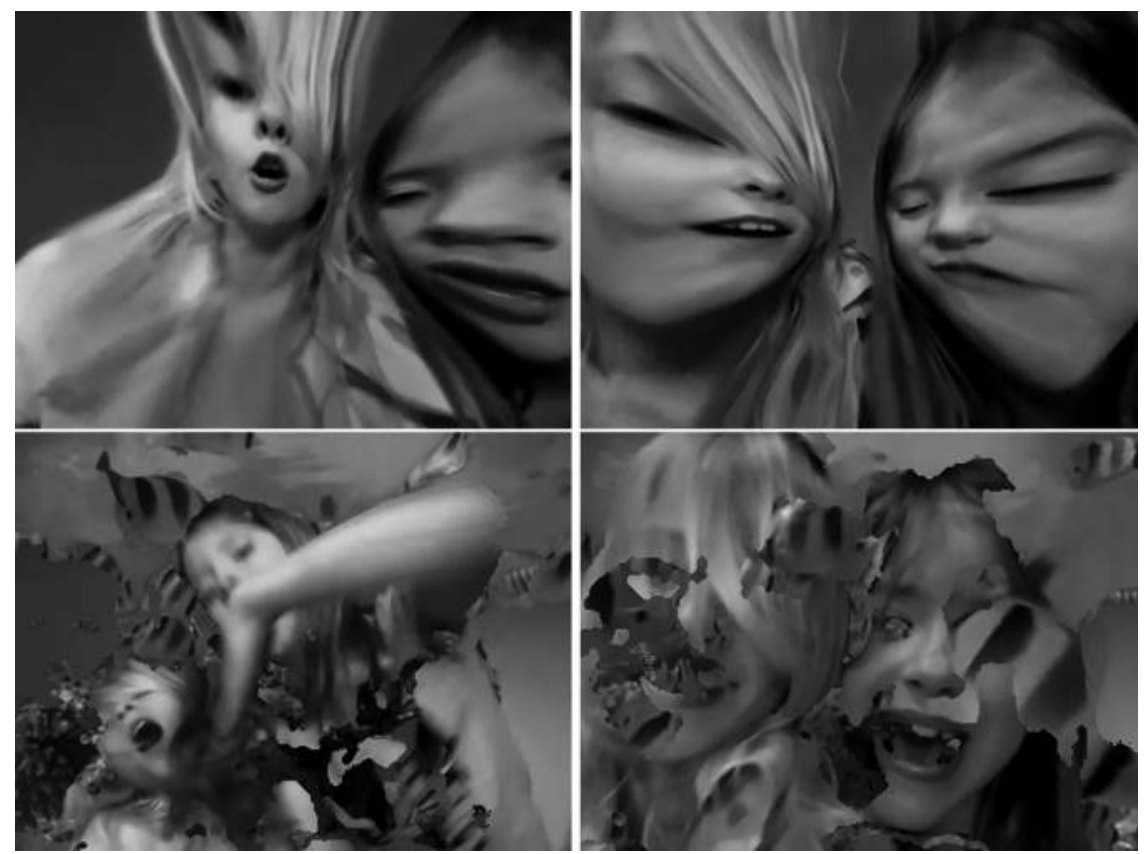

Figure 2.1 Ugly giants, electric eels and shark-human-cats

\section{Children}

The word 'children' in this book's subtitle would seem at first glance to be the least ambiguous, the most self-evident. However, the notion of the child as a distinct entity both biologically and socially is historically recent and culturally varied. For Ancient Greece, and the European cultures it influenced, 'distinguished children socially from babies and adults if only as objects of aesthetic appreciation', and 'children did not emerge as social entities' in the West until the early seventeenth century (Stone 1971, 5). And,

across different cultures children are looked after in periods of dependency in a variety of social arrangements, are cultural participants in varying degrees of inter- and intragenerational segregation and integration, and occupy the status of 'child' for varying lengths of time. (Hutchby and Moran-Ellis 2001, 1)

Before the seventeenth century, it seems, there were only babies and adults. Once babies were out of their swaddling clothes they were dressed in small versions of adult clothing, and play and festivals were not differentiated by age groups. 
No doubt children played together, but there was no 'children's culture' as such. Gregory Stone notes that the invention of childhood as a time characterized by play (and by implication, the child a being primarily characterized by play) was established with the rise of entrepreneurial capitalism in Europe and its attendant moralism, 'and pari passim, children were established as identifiable social beings' (Stone 1971, 6).

Through the nineteenth and early twentieth centuries, children in the industrial world were incrementally removed from the (now) adult worlds of work: first the offspring of the bourgeoisie, then - with new legal frameworks and the introduction of universal education - working-class children too. Differentiated from adults socially and discursively as 'school children', they also became positioned, before long, as consumers of a commercial children's culture of books, toys and games (see Chapter 4, 'Media Worlds').

To oversimplify, by the twentieth century, children in the developed world were constituted by four overlapping worlds: as school pupils they were to be defined now not simply as juvenile, immature or 'not-adults', but increasingly as works in progress - always at a certain stage of learning and development and always short of the next stage or level. Play has shifted from by and large ignored behaviour that took place when adults were not placing demands on children's time to an ambiguous process that might hinder intellectual and moral development with its trivialities and animal passions, or support and edify it through imagination, cooperation, rules and the disciplines of training and practice. This ambivalence remains evident today. Another effect of school is to establish clear distinctions - through organization of school years, classes and shared playtimes - between children according to the year in which they were born. ${ }^{1}$

Secondly, the child has become a more domesticated being - as social and cultural life became increasingly oriented towards the family and home through the gradual 'mobile privatization' of everyday life in the twentieth century (Williams 1974). Thirdly, and closely bound up with the domestication of childhood, children have become consumers in a new commercial toy and media culture, with its ever more nuanced rendering of demographic categories from the 'pre-school' to the 'tween'.

Fourth, children are sometimes boys and girls. In Bruegel's paintings of street play in the sixteenth century boys and girls are barely distinguishable, whereas in contemporary representations in television advertising they appear to be different species - they are certainly rigidly colour-coded as blue or pink from 
before birth. Some observational studies of young children's play note minor differences in play tastes between boys and girls as well as overlaps in preferences for certain types of toy or make-believe scenarios (e.g. Herron and Sutton-Smith 1971, 79), whereas much recent work on children's culture has focused on the construction and reconstruction of gender through toys, games and video game culture (Alloway and Gilbert 1998, Cassell and Jenkins 1998, Walkerdine 2007). Children's access to computers and video game consoles, and the kinds of play they conduct, is framed and shaped by the cultural and discursive frames of the family, school and commercial culture. However, boys and girls also play together, and alongside each other in the playground or with siblings at home. In the small studies of moments of the flow of gestures and images in performative or imaginary play, gender roles are sometimes less clearly drawn than in children's material and media. Moreover, as I will argue in Chapter 7 ('Real Worlds'), the semiotic and behavioural materials of play are rarely straightforwardly imitative or representational. Play inverts, turns the world upside down. Media identities and roles can turn out to be not only constraints but an armature or scaffolding for new hybrid or emergent performances.

All this said - and lastly - children always did, and still do, play beyond the scrutiny and structure of well-meaning adults, inside and outside, often with siblings and friends of different ages, in a world both spun out of nothing in the moment and also shot through with jokes, games and songs that can be traced back over decades and centuries: an oral and gestural repertoire documented across generations by Iona Opie (Opie 1993).

The division of children into levels or age groups by educational and psychological theories establishes a temporal taxonomy of development. This book is not concerned with development in these terms, and it refers to play events involving children from infanthood up to the mid-teens. This is in part a function of pragmatics or personal history, in particular the various points and trajectories of coincidence of the ages of my own family with my own research contexts. Alex and Jo were aged three and four when I began, and they are fourteen and sixteen as I write this book. In part, though, it is a function of the particular aspects of play that seem most appropriate for engaging with playful digital media culture: the imaginative, make-believe, performative and transformative play that characterizes children's behaviour from infanthood to the approach of adolescence. Moreover, whilst it is concerned with moments of play - not its 'development' nor that of its players - the book is interested in the temporality of play. Gameworlds extend in time as well as space, and even 
ostensibly fragmentary or discrete moments of play can prove to be simply one surfacing of a twisting, changing thread of images or behaviours, whether the evolving playground song that Iona Opie hears in the same playground but decades apart, or the repetitions of a particular child or group of children over days and across space - sustained by imaginative investment (the reiteration of favourite characters or animals in performance or drawing, for instance) or through the mnemonic augmentations of media technologies (repeated DVD or YouTube viewings, progression through the levels of video game). To use a concept from philosophy, the book is more concerned with children and media in their 'becoming' than in their 'development' (Deleuze and Guattari 1997). Reading Iona Opie's funny and astute accounts of playground activity, I was struck by one small boy who pops up by Iona Opie's elbow over several weeks. With his obsessive imagination and his intense desire to know about, and evangalize for, gorillas, he is not developing into an adult, but rather becoming-gorilla:

\section{Thursday 7 th September}

[...] The gorilla boy was still there. 'Elephants are not very strong', he said. 'D'you know how a gorilla walks? Stamp'; and then, wistfully, 'I wish gorillas could talk. [ ...]

\section{Monday 25th September}

[...] 'Who's supposed to be on it now?' they called to each other, giggling. 'I think it's Nicky'. It seems that the basic games, though not 'special', are as good fun as ever.

The gorilla boy put his arm in mine and assured me that 'Gorillas can lift you up. It is as if he is in love with gorillas; their name must always be on his lips.

The craze for miniature cars is still going on [ ... ]. (Opie 1983, 80, 85)

It is worth noting here that the gorilla boy isn't becoming a single gorilla with a name or gaining individual features from, say, a cartoon or natural history documentary. He is becoming gorillas, and this multiplicity across species and beyond individuals, 'interkingdoms', is of particular interest to Deleuze and Guattari (Deleuze and Guattari 1998, 266-267). It is a distinctive aspect of imaginative play too, and I'll return to it in Chapter 7.

Finally, the theoretical concerns with which I approach the observation and description of video game play events are concerned with undermining ostensibly fixed distinctions between the human and the non-human in contemporary technoculture. The collaged human/synthetic images on the laptop screen as the computer fish, camera and girls play together are a visual 
metonym of the hybrid state of everyday life in digital culture. Children are born into an environment replete with digital media technologies, play with computers and tablets before they can talk, are looped into cybernetic circuits of play and communication at home and at school. These conceptual frameworks are sketched out in the next chapter and underpin the descriptive work throughout the book. For now, I will note that children are no less monstrous than all the cyborgs and posthumans that populate contemporary technoculture (Davis-Floyd and Dumit 1998).

\section{Play}

Playfulness is a volatile, sometimes dangerously explosive essence, which cultural institutions seek to bottle or contain in the vials of games of competition, chance, and strength, in modes of simulation such as theater, and in controlled disorientation, from roller coasters to dervish dancing.... Most definitions of play involve notions of disengagement, of free-wheeling, of being out of mesh with the serious 'bread-and-butter', let alone 'life-and-death' processes of production, social control, 'getting and spending', and raising the next generation .... (Turner 1982, quoted in Schechner 1998, 4)

There is no agreed definition of the word 'play'. As well as referring to the pleasurable activities of children and adults when not constrained by work it can also denote any free movement or latitude in human, animal, mechanical and natural bodies, from the play of light to the play of gears in an engine. It seems easier to list and categorize play rather than define it. For instance, Gordon Burghardt cites a recent study of US kindergarten play that categorizes types of play: 'large-motor play, small-motor play, mastery play, rule-based play, construction play, make-believe play, symbolic play, language play, and risktaking play' (Burghardt 2011, 10). These are not mutually exclusive, and I would suggest that any particular moment of play would involve a number of these so the whole taxonomic exercise is largely unhelpful. Indeed, as I suggested in the introduction, it is equally important to explore and trace the movements of play within and between games, to note the productive tension between fluidity and flexibility and rules and structure:

The game consists of the need to find or continue at once a response which is free within the limits set by the rules. This latitude of the player, this margin accorded to his action is essential to the game and partly explains the pleasure 
which it excites. It is equally accountable for the remarkable and meaningful uses of the term 'play', such as are reflected in such expressions as the playing of a performer or the play of a gear... (Caillois 1962, 8).

I have touched on some significant concepts and thinkers for the study of play and games, and they will be developed throughout the book. I have found it useful to develop an approach to play and games from an ethnographic tradition of cultural enquiry and not through any attempt to establish formalist definitions or taxonomies of types of play, players and games.

The emphasis in both Huizinga and Caillois' thought is on play as a voluntary activity, separate from work and mundane life, materially unproductive, an activity of 'pure waste', but characterized by intense absorption on the part of the player:

... the domain of play after all constitutes a kind of islet, artificially dedicated to calculated competition, limited risks, inconsequential make-believe, and meaningless panic. (Caillois 1962, 85-86)

This conceptualization works better for adults than children. Most of the waking life of an infant or young child is concerned with playing: playing with sound and language, the child's own body, food and any object within reach becomes a toy. Important theories of child psychology are predicated on the fundamentally playful way that the infant negotiates his or her emergence as an individual in the world (Winnicott 1974). The more enlightened approaches to education over the past century or two are underpinned by the belief that learning is most effective through play, whereas bored children (and adults) play during work, undermining the work in hand through whispered jokes, written notes (via paper or SMS), surreptitious missiles and so on. All of these everyday events are brought about by the tensions between play and more instrumental activities and behaviours, but in none can play and work be clearly mapped as separate domains.

Even the voluntary nature of games and play for older children is open to question. Involvement in sport organized by adults can be more or less coerced, as can involvement or exclusion from games by groups of children. Play can be cruel and bullying. Moreover, if, as many studies in psychology and animal behaviour assume, play is a fundamentally natural phenomenon, shared by many other species from the higher primates, other mammals such as otters, dolphins and domestic pets to some birds, then it must be driven at some level by instinct, 
before its instantiation in any particular voluntary game (see, e.g., Bruner et al. 1976, Pellegrini 2011). Theorists of animal play argue over whether young animals are training themselves for essential adult behaviour, such as predation or the ritual combat of mating, whether play is the expenditure of redundant energy when the animal is well-fed and not facing imminent threat or whether it serves a primarily social function - building and sustaining relationships and hierarchies. Herron and Sutton-Smith are suspicious of the application of this first theory, 'adaptive behaviour', to human children's play:

We are accustomed to viewing adaptive behaviour within a utilitarian 'survival-' or 'achievement-' oriented context. Whereas it may be that the intrinsic function of play is only very indirectly related to any of these things, it would appear to have more to do with whatever satisfaction comes from making one's own unique expressive response to one's own experience. (Herron and SuttonSmith 1971, 2)

Though games are a more bounded category than the play that brings them into being, they too are an ambiguous and undertheorized phenomenon, particularly within the study of media cultures. Games are at once cultural practices (even rituals), media/aesthetic objects, toys and social (or solitary) events. Again, game studies is developing rich conceptual and analytical resources for identifying the particular forms and codes of games, from taxonomies of types of games and play to the analysis of rule sets in video and computer games (e.g. Walther 2003).

For Avedon and Sutton-Smith, games are

an exercise of voluntary control systems in which there is an opposition between forces, confined by a procedure and rules in order to produce a disequilibrial outcome. (Avedon and Sutton-Smith 1971, 7)

This definition probably applies to most playful events called games, from cards to football, and its identification of control as a salient characteristic will be explored later in the book. Its emphasis on a clear end - both temporal and agonistic - is shared in influential definitions in game studies, notably Jesper Juul's analysis of winning states, or 'variable and valorized outcomes' (Juul 2003). However, whilst many games have definite winning states, there are many structured events of play which do not yet are called games by their players: young children might call any more or less sustained and coherent engagement with characters, actions or environments a game. These imaginative collaborations may well not have a competitive element nor a clear end point, 
but they have enough of a symbolic theme or complex for the children to feel they are generating a gameworld. Moreover, if a descriptive rather than taxonomic approach is taken to the study of games in everyday life, even games with clear rules and agonistic motives may still not - in particular events of their playing - ultimately generate a competitive relationship between their player, nor a fixed winning state. For example, a 'game' of badminton or tennis can easily be adapted to a pattern in which the players are concerned to keep the shuttlecock or ball in play rather than trying to score points by hitting it out of play - we might wonder what the ratio is between games of Monopoly begun, over a Christmas holiday for instance, and games of Monopoly actually finished and won. For adults or children, or adults and children, formal games are often used as structure or scaffolding for social activity, rather than as an agonistic end in themselves.

The structuring and directing of playful behaviour through rules is a key factor in analyzing games. Within psychological research on children's play, games and playful behaviour are categorized by the relative rigidity, flexibility or negotiability of rules:

A main characteristic particular to games is that they involve rules and roles that have previously been established by others, are probably codified in some way, and thus can be explicitly communicated. The existence of rules and roles places constraints on children's behaviour within the game. These rules are much less flexible than the implicit rules and roles evident in peek-a-boo, rough and tumble (R\&T) play, and those negotiated in fantasy play.... (Baines and Blatchford 2011, 262)

Games, then, are 'social objects' that can be planned with appeals to tradition and precedence with new forms emerging through negotiation or experimentation. 'Cops and robbers' might simply take the form of a chase, or it might be shaped by fantasy and role play.

It is often assumed that a game must be social, a definition that rules out games with non-human players (i.e. video games), but also earlier singleplayer games (notably card games in the solitaire family), or - less obviously games in which the 'rules' are not so much rules as laws embedded in the play environment, for example gravity, the arrangement of wall and playground for marbles and balls and the dynamic geometries of rope in skipping games (Opie and Opie 1997). As we'll see later in Chapter 5 ('Soft Worlds'), the 'rules' of a video game might be more usefully thought of as gameworld 'laws', built into the virtual environment. 


\section{Virtual}

Let's return to the fishy world of the twins and Photo Booth, with a continuation of the passage by Victor Turner quoted above:

Play can be everywhere and nowhere, imitate anything, yet be identified with nothing.... Play is the supreme bricoleur of frail transient constructions, like a caddis worm's case or a magpie's nest.... Its metamessages are composed of a potpourri of apparently incongruous elements .... Passages of seemingly wholly rational thought jostle in a Joycean or surrealist manner with passages filleted of all syntactical connectedness. (Turner 1983, quoted in Schechner 1988, 4)

The girls found a feature that adds a photographic or video backdrop to the self-portraits. Lily improvized a rap-like performance about the Eiffel Tower against a Parisian scene, and then, joined by Josie, settled on an animated scene of tropical waters and fish. They soon found that the software assumed or required - in an echo of the very early days of portrait photography - a static subject. Otherwise, it could not adequately trace the edges of the human head and body and accurately map the pre-rendered background as if behind them. With their movement the real-time video image of the twins was broken up, mixed up and overlaid with the bright tropical waters in an animated collage. This virtual immersion inspired the girls to sing (Figure2.1):

J and L (with swimming actions): We're swimming in the fishtank (they push each other and giggle)... We're in Penny's house, and we are in the kitchen, and we are swimming even though we're on the ground!

J: And I might swallow you, fishy!

J and L: Because I'm half shark (Lily lunges towards the webcam with exaggerated biting gestures and noises)

(They sway from side to side together gracefully)

J: We are a shark and human put-together-to-make-a-cat (these last words spoken quickly as the rhythm of the song is dropped)

(The song picks up again)

J (with Lily picking up on the last drawn out word of each line): We're happy to be eating f-i-i-i-i-sh (they make smiling faces by pulling up the corners of their mouth with their fingers), even if, they're electric e-e-e-e-ls.

J: I'm gonna electrocute them!

(The song degenerates again into pushing, swaying, biting motions and snatches of singsong references to fish.) 
The term 'virtual' is particularly ambiguous, but it is crucial for addressing the behaviours and bodies described in this book, and will be discussed in more depth in Chapter 7. In the context of digital media, it can function as a loose shorthand for anything to do with the intangible operations of computer technology, and particularly for the immaterial spaces of communication and play these operations generate. I have already used it as such to describe the computergenerated simulation of Lego vehicles. It generated excitement around actual and predicted technologies of virtual reality (VR) in the late 1980s and 1990s, promising to realize what the VR enthusiasts saw as an essential trajectory of the history of media technologies from cave paintings to Renaissance perspective, photography to cinema: an arc of technical evolution towards ever-greater immersion, a paradoxical disappearance of the mediating technology through its own sophistication:

Virtual reality is immersive, which means that it is a medium whose purpose is to disappear $[\ldots]$ transparent, perceptual immediacy, experience without mediation. (Bolter and Grusin 1999, 22)

The term 'virtual', then, suggests a phenomenon or entity that is not quite real. This 'not quite' could indicate something that has the potential to be real or will soon be real, in the sense of a task that is virtually complete; or it could be something that seems real, but may never become so, the worlds of VR applications, for instance. Lister et al. point out that for all its ontological instability, a VR world or other computer simulation is quite definitely real in a dual sense:

Without these machines, there would be no simulation. Therefore, simulations are importantly real by virtue of the technologies necessary to producing them, and the effect they have upon us. (Lister et al. 2009, 389)

The nature of the virtual reality of a video game world as computer simulation, and the nature of the effects this virtuality has on players, will be examined throughout this book, particularly in Chapter 5. As the initial hype around VR faded, it became apparent that for all the new possibilities for VR as an immersive media form (recently revived in video games with devices such as Oculus Rift), predictions of immediacy - in Bolter and Grusin's sense of the dissolving of the mediating apparatus - did not stand up to users' experiences of cumbersome headsets, motion sickness and lag between their movement and the virtual world's response. The 'virtual' persists as an adjective for a range of everyday digital media, however, from the numerous 'virtual communities' of 
Internet media to online 3D worlds, and it overlaps with concepts of cyberspace and simulation. William Gibson's 'cyberspace' - a fictional future of immersive datascapes - was regarded as the prescient template for $\mathrm{VR}$, but is now more commonly applied to the communicative networks of the Internet, particularly email, social media networks and forums. 'Simulation' is a key term for both theories of play and computer media worlds, and it will be addressed in detail in Chapter 5.

Additionally, using the concept of the virtual in this sense familiar from popular digital culture and technological research, Lister et al. articulate it with an older sense of the virtual from philosophy. Drawing on Gilles Deleuze, this virtuality insists on the temporal dimension of reality: there is a reality to things that do not (yet) exist. Like the task that is virtually completed,

[t]he reference to time is clear: the task's completion is just about upon us, but is not yet. In this sense, a reference to the virtual includes future states as part of the real; the future has a kind of reality which is virtual, but not actual. This is the sense of the 'virtual' that Gilles Deleuze maintains: the virtual is real, but inactual. That is, it has real existence but not in the same way as the things that are actually around us. (Lister et al. 2009, 389)

A proper examination of this sense of the virtual is not within the scope of this book, but I would highlight three aspects that might usefully inform the study of everyday play. Firstly, it draws attention to the media technological platforms that occupy so much of children's time and attention today - VR as an everyday, here and now, reality. Secondly, it emphasizes the real operations and effects of phenomena that are inactual. Together with the processes and configurations of software in digital play and communication, we might also include the intangible and immaterial - but still real - affectual realms of media images and dramas, and the building of imaginative worlds across individuals, groups and environments in playful events (see Chapter 6, 'Playgrounds'). Thirdly, it highlights the temporal and emergent dimension of play events - both the virtual time of video game worlds and the micro-evolutionary becomings of imaginative play. Rob Shields' explanation of the philosophical virtual evokes something of the fluid and creative dynamic of playful events:

The Virtual itself can be said to be a capacity to be actualized as a singular, concrete object. Actualization is performative - the Virtual itself is a multiplicity which can be actualized in different ways. If it is known by its effects, then it is known through a specific instantiation, not as a whole. It thus retains its 
creative character as an ontological category pertinent to discussions of change, becoming, genesis, development, emergence, autopoiesis, the genetic power of codes as well as of codings themselves. (Shields 2006, 285)

Brian Sutton-Smith frequently refers to the imaginary dimensions of (nondigital) games and playing as 'virtual':

In solitary or private play children create cultures of play that are virtual worlds not mundane worlds, and often with not much obligation to the latter. (SuttonSmith 1997, 155)

Again there is the sense of an affective reality that sits within, or opens up from, everyday reality, but that is of a different order in its microcosmic vividness. Like the simulated world of the video game it is a fabrication, but synthesized from different materials. All these senses of the virtual overlap and it is not my concern here to clearly define and delineate them. One important point to note is that in all these senses of the term, the virtual and the actual are both real.

\section{Media}

The words 'medium' and 'media', are also polysemic and unstable, even when we are only referring to the technologies, practices, aesthetics and meanings of information and entertainment media. In his book Understanding Media, Marshall McLuhan provocatively included chapters on cars and weapons alongside those on television and typewriters (McLuhan 1964). When used in general conversation, 'the media' tend to refer to professionally produced and distributed information, news and entertainment channels (TV, radio, newspapers, cinema). Media studies has generally focused its critical and analytical attention on these media's transmission of messages or ideological shaping of public and private discourse via a circuit from producer to consumer through the economies and technologies of distribution, the ideological interests of dominant class and gender formations. The emphasis on the message, whether news item or cinematic representation, assumes both a fundamental distance in time and space (and social position and power) between producer and consumer, and that the salient operations and effects of the message are mimetic or representational. The task of media criticism, then, is to point out the discursive mechanisms through which encoding takes place, and to decode the meanings of a message (or to observe people in their everyday media cultures 
decoding, accepting or negotiating these meanings) (Hall 1997). These circuits of signification are complex, shifting and entail various channels via which media consumers work with or feed back on their mediated lives.

Roger Silverstone suggested it might be more useful to think in terms of mediation as a dynamic process rather than media as discrete platforms and channels:

Mediation involves the movement of meaning from one text to another, from one discourse to another, from one event to another. It involves the constant transformation of meanings, both large scale and small, significant and insignificant, as media texts and texts about media circulate in writing, in speech and audiovisual forms, and as we, individually and collectively, directly and indirectly, contribute to their production. (Silverstone 1999, 13)

What help does this central concern with meaning offer to the study of the often nonsensical and meaningless worlds of children's play, now so tangled up with the images and technologies of popular media? We can, of course, look for the meanings and the representations in commercial children's culture, in Disney for instance (discussed further in Chapter 4), but this would be to ignore perhaps the most extensive and intensive of children's media experience. For example, I will later refer to a study of primary school girls singing and dancing to Beyoncés 'Single Ladies'. We might analyse the song's lyrics and its representation of gender relations, or the song's video with its assertive sexuality. But how do we describe the music as a media form through its rhythm, harmonies, tone, etc., and particularly important for the study of play, how its aesthetic form acts on the players' bodies, how it suggests or compels dancing, singing and performance by individuals and groups? Girls' playground singing games pick up and shred media 'messages' such as pop songs, recompiling them into nonsensical rhythms and rhymes: affectual devices for establishing and synchronizing moments of collective pleasure. Meaningless but deeply significant in children's everyday lives, closer perhaps to McLuhan's assertion that the medium is the massage, the significance of media technologies or technics (including bodily techniques of rhythm and dance) exercised through the orienting and pressing in on bodies rather than communicating any particular story or theme.

Against the notion of mediation as the transmission of messages over distances of time and space we can posit other models of communication. Jackie Marsh suggests that in its performativity, use of props or artefacts and its repetitions, children's media play can be seen as akin to ritual, with children 
creating 'social performances in which they are the pivotal character' (Marsh $2005,42)$. Ritual and play unfold in an intense, proximate space and time, they are of the moment and their effects localized, generated in and through the participants. This expansive definition of ritual reaches from the formality of religion and the sacred to the minutiae of everyday life, 'ritual can be recognized as performance which is habitual' (Marsh 2005, 41). For James Carey, the ritual view of communication is no less important than transmission. As ritual, communication is 'a symbolic process whereby reality is produced, maintained, repaired and transformed' (Carey 1992, 23).

If the sacred nature of ritual seems too formal, and possibly culturally conservative, to fully account for the fluid and improvisatory nature of children's play, it can be linked to contiguous and overlapping profane cultural formations such as carnivals and festivals. Huizinga sees play itself exercised through ritual up into the structure of civilization, but also as a productive degeneration of ritual forms and practices; for example, the labyrinth of the monk's symbolic pilgrimage has become the child's hopscotch matrix. Anthropologist Victor Turner makes a key distinction within ritual cultures of performance. Play, religion and ritual all occupy liminal zones within cultures (often mapped onto or enacted within liminal space as well), but whereas liminal practices such as initiation rites reinforce and reproduce a social order, there are also liminoid zones within the developed world that have more potential for disruption and the generations of new cultural forms and behaviours. This model of play and cultural production has been usefully applied to video game culture by Jon Dovey and Helen W. Kennedy:

The player returns from the liminal ritual space with a renewed sense of his or her place in the established social order. Liminoid phenomena, on the other hand, are much more individualized and commodified. They develop 'apart from the central economic and political processes, along the margins, in the interfaces and interstices of central and servicing institutions - they are plural, fragmentary and experimental in their character.' (Dovey and Kennedy 2006, 34-35)

In the next chapter, I will open up this question of the relationship between the micro-world of the ritual or play event and the material and symbolic environments within which they form. A theory of communication as ritual captures something of the distinctive space-time and character of games, but these gameworlds are not closed systems. 


\section{Virtual media}

For all the ambiguity and openness of the terms 'virtual' and 'media' as set out earlier, I use the term 'virtual media' rather straightforwardly to refer to popular entertainment and communication media on digital platforms and which generate their own time and space for engagement, interchange or play. My main interest is in video and computer games, but these extend into other playful digital formats such as social media networks, mobile phone play and apps for mobile and location-aware devices, and are often found within the broad mediascapes of commercial transmedia systems (see Chapter 4), or accessed through managed network services such as Xbox Live. There is no longer a clear distinction between the terms 'computer game' and 'video game'. Until the early 1990s (with the dissemination of the personal computer as a consumer product), 'video games' were produced for a video game console or dedicated device whilst 'computer games' were produced for home computers. The latter were often less sophisticated graphically but afforded the player the opportunity to intervene in the game software and make changes to the appearance or structure of the game. Over the past few decades, the popularity of personal computers in the home has led to the rise of PC gaming and a marked overlap in the games available for consoles and PCs.

\section{The everyday}

The concept of everyday life is central to work on the social and cultural locations and operations of technology, media and children's culture (Highmore 2002). Media studies, cultural studies, sociologies and anthropologies of technology all address the public, work or domestic relationships, routines, cultural practices and spaces through which people, including children, make, and make sense (or nonsense) of, the world (e.g. Akrich 1992, Mackay 1997, Mackenzie and Wajcman 1999, Holloway and Valentine 2000, Hutchby and Moran-Ellis 2001, Facer et al. 2003, Flynn 2003, Michael 2006, Weber and Dixon 2007). Everyday life is the site in which the popular instrumental and ludic uses of digital media are negotiated, played out and invented, and it is in the realm of the everyday that spatio-temporal limits, restrictions and power relationships are reinforced or opened up through technologies. The home and households are a primary 
target for the producers and marketers of media objects and platforms and hence the lived practices, experiences and techniques within which these artefacts are engaged with and consumed.

I will return briefly to the Photo Booth performance to set some of these terms into play. It was a tiny event, the girls played with the laptop for around ten minutes, with the fishy theme lasting less than two. The space of the gameworld extended in to the laptop itself, its camera, software, RAM and storage, and out to little more than a square metre of bodily movement. However, I could have described it in much more detail than I have discussed here. It was not a complicated game, but it was complex - a complex: it brought together patterns and themes, it organized diverse physical, virtual and semiotic material into an event that was nonsensical yet with its own patterns, rhythms and playful logic. There was no centre as such to the event, it couldn't be said that the PhotoBooth software generated the game, nor the camera, the animated fish, the computer or even the children. They brought themselves together, images, sounds and actions, human and non-human, familiar routines of singing and performance and new routines of video feedback, collapsed space and visual artefacts.

As already noted, the place or space in which this game event takes place is ambivalent. The girls are dancing in front of the screen and camera, so we might draw a map shaped something like a beam of light fanning out from the plane of the laptop screen. The field of vision of the camera marks the edge of the playing field so the spatial boundary is not drawn in actual space but is enforced by visual feedback: simply, a girl knows she is out of the game when she moves off the edge of the image on the screen. The webcam, a barely visible $3 \mathrm{~mm}$ diameter disc above the computer screen, defines the possible actual space, whereas the feedback of the video signal, looping through the human lenses and motor systems, regulates it. But the playspace is also on the screen: a dynamic but flat surface of computer animation and live video, torn up and overlaid. These loops of information and feedback are processed invisibly as well, both in the hardware and software of the laptop and through the imaginations and sensorimotor systems of the children. And the familiar games of mediated domestic performance, established in the twentieth century with domestic photography and later home video, are newly mediated and concentrated by the webcam and real-time video feed.

The multiplication of space across the actual and virtual is felt and lived by the children, so much so that they can sing it: 'We're swimming in the fishtank... We're in Penny's house, and we are in the kitchen, and we are 
swimming even though we're on the ground!' The technological artifice of this simulated ocean environment is alluded to as well: these fish look and swim like ordinary tropical species, but they also electrified, 'electric e-e-e-ls'. This adds a new cyborgian dimension to the more familiar monstrous hybrids of nonsense play, here the half-human, half-shark that somehow adds up to become a cat.

These little descriptions indicate much that will be explored throughout this book. A key concern is the role of digital technology and play in generating confusions or renegotiations between usually distinct concepts in our understanding of children, technology and media: in everyday life and media culture - the new with the old; in the use of computer media - virtual with actual space; in the moral critique of popular culture - passivity with agency.

\section{Note}

1 Studies of child development establish broader developmental stages. For example, one possible structure of periodicity is that set out by Elizabeth B. Hurlock: babyhood 0-3 yrs; childhood 3-6 yrs; youth 6-11 yrs; adolescence 12-21 yrs (in Herron and Sutton-Smith 1971, 52). 



\section{Microethology: Methods for Studying Gameworlds}

This chapter will set out the methods I use in studying play, the conceptual enquiry that underpins these methods, the theoretical and empirical traditions that situate this work and some of the practical considerations it raises. It will address the methodological demands of studying the relationships between the virtual and the actual, cyberspace and domestic space, in everyday popular digital culture. The book as a whole attempts to identify and theorize both human and non-human agents in the moment or event of gameplay: so the object of study here is neither human players nor dynamic software but what happens when they come together to generate an event of gameplay.

\section{Ethnography}

My approach to empirical research is in some key respects part of an interdisciplinary tradition of small-scale ethnography, particularly studies that describe cultures or subcultures within the Western or developed world - that is participant observation not of exotic or pre-industrial others, but groups with which researchers have some familiarity. Media and cultural studies have their ethnographic strands, with attention to television audiences (Ang 1985, Morley 1992) or to youth culture (Willis 1978, Hebdige 1979). To overgeneralize, these studies were primarily concerned with the discursive and identity-forming processes of media consumption or everyday performance. However, in this book I am less concerned with the meanings constructed around children's media culture and more concerned with the materiality of both new media and the lived experiences of their consumption or playing. The 
media technologies themselves (both hardware and software) and their agency as machines (or bodies in play) are of concern, not just their transmission of images and scenarios.

The case studies used in this book, those written by myself and those by others, are very much concerned with describing the experiences and texture of everyday culture; they are descriptive and interpretive and not scientific in their aims. Clifford Geertz has described the potential for ethnography not only to 'reduce the puzzlement' we face in encounters with different cultures, but also in the study of more familiar worlds in which it can usefully induce puzzlement:

[...] displacing the dulling sense of familiarity with which the mysteriousness of our own ability to relate perceptively to one another is concealed from us.

(Geertz 1993, in Hine 2000, 64)

I am personally interested in, and influenced by, a stream of empirical engagement with the everyday that can be traced back to the Surrealist movement of the 1920s. Surrealist writers and photographers in particular regarded the mundane everyday world not as a static environment against which everyday life took place, but rather as a realm shot through with the marvellous, a strangeness that can be revealed through playful and poetic activities and attention:

Surrealism is not just a technique for making the ordinary extraordinary; the everyday in Surrealism is already strange (it is collage-like). In Surrealism our drab habits of mind understand it in this way. Instead the everyday is where the marvellous exists. (Highmore 2002, 46)

Thus we could 'read Surrealism as a form of social research into everyday life' (Clifford 1988, Highmore 2002, 46). This Surrealist stream was fed by, and into, critical thought and anthropology in France that was concerned with social and cultural phenomena that can broadly be connected with play. Marcel Mauss' work on pre-industrial gift economies was influential on Georges Bataille, who worked with Roger Caillois on the study of sacred and ritual cultures (including aspects of everyday Parisian life), and on the Situationist International in the 1950s and 1960s, who were also avid readers of Huizinga and saw play as a transgressive revolutionary force (Giddings 2007c). The Surrealist ethnographic attention to the intangible forces of desires, dreams and the unconscious - as well as the ambiguous but powerful persistence of spaces of the sacred in the modern world - offers aesthetic and conceptual resources for the operations of virtual media, imagination, play and non- 
human agency in the every day. An ethnographic surrealist sensibility, then, can address not only the marvellousness of the adult, urban, environment, but also the inherent surrealism of children's play. Consider this account from a book on child development, for instance:

The dolls seem to take our lives of their own as children create devices to create the illusion that the dolls are making decisions and reacting to events within their own autonomous story world. Furthermore, in speaking about for their dolls, children come to speak about things that are not visible, such as the thoughts and feelings of the dolls and the objects in the story world that are not part of the setting in which the story being told. By the end of the preschool years, children often can create entire autonomous story worlds, complete with characters facing problems and resolving them 'on their own' in scenes that are quite fantastic and distinct from the actual scene in which the play is taking place. (George et al. 2005, 62)

Children's play brings the inanimate to life, collages together disparate material and semiotic fragments and effects all kinds of syntheses and transductions. It is utterly everyday, but marvellous and phantasmagorical (see Chapter 7, 'Real Worlds').

I draw also, therefore, on a range of theoretical and methodological resources that are critical of the humanist assumptions of the humanities and social sciences, and that suggest ways for studying the everyday world as technocultural, constituted by both human and non-human bodies and forces. These include science and technology studies (STS), actor-network theory (ANT) (and the work of Bruno Latour in particular), Donna Haraway's work on science and cyberculture and the realist philosophy of Gilles Deleuze and Félix Guattari. This book isn't the place to set out or synthesize these diverse bodies of thought and practice in any detail, ${ }^{1}$ but I will pick up and use ideas and approaches from them when useful throughout. For the purposes of the book, underpinning them all is a fundamental questioning of fixed discursive and methodological separations of, variously, the human and the technological, the natural and the cultural and the material, and the imaginary in culture.

\section{Anthropology of technology}

Broadly, then, I have been influenced by a range of approaches that might be loosely bracketed together as an 'anthropology of technology': 
...technical objects and people are brought into being in a process of reciprocal definition in which objects are defined by subjects and subjects by objects. It is only after the event that causes are stabilized. And it is only after the event that we are able to say that objects do this, while human beings do that. It is in this sense, and only in this sense, that technical objects build our history for us and 'impose' certain frameworks. And it is for this reason that an anthropology of technology is both possible and necessary. (Akrich 1992, 222, my emphasis)

The problems and possibilities here are both terminological and actual: how to study the lived nature of humans and objects in digital culture? An emergent 'cyborg anthropology' (Downey et al. 1995), or 'cyborgology' (Gray et al. 1995), would at least need to interrogate the humanist assumptions of both the cybernetic organism and the anthropos.

Actor-network theory contributes to this anthropology, or sociology, of technology. Mackenzie and Wajcman introduce it as identifying 'the reciprocal relationship between artefacts and social groups' (Mackenzie and Wajcman $1999,22)$. However, ANT draws in a far more heterogeneous array of agents or actors as constituent of sociotechnical networks:

If human beings form a social network it is not because they interact with other human beings. It is because they interact with human beings and endless other materials too [...] Machines, architectures, clothes, texts - all contribute to the patterning of the social. (Law 1992)

A study proceeding from an actor-network theory position, then, would assume no primacy of agency between humans and non-humans (whether artefactual, scientific or natural) in any network, phenomenon or system, implicitly or explicitly rejecting the humanist worldview of the humanities and social sciences. The implications of this are far-reaching and go beyond the nuances of the effects and determinations studied as SST (social shaping of technology). It questions engrained conceptual distinctions between nature and culture, humans and artefacts, subjects and objects:

Both society and technology, actor-network theory proposes, are made out of the same 'stuff': networks linking human beings and non-human entities. (Mackenzie and Wajcman 1999, 24)

For Bruno Latour, then, society has always been an indissoluble network of technological and human entities: society is (and always has been) constituted by humans, machines and tools. 
The technocultural circuits of the video game, then, are only one, particularly intense and intimate, network, but one that consists of material and technical, aesthetic and embodied connections:

Digital games are objects that exist in the world; however much their digital virtuality is celebrated they are enacted and produced in strikingly visceral ontologically virtual - ways. The noise that a PS3 game makes when it is pushed into the blu-ray drive, the cables over which Xbox LIVE arcade games are downloaded, and the wars and environmental depletion taking place to produce the coltan-based batteries in your wireless devices [... ] all suggest a materiality to gaming that links digital games to the world and demands that they are also understood as objects in the world. (Apperley and Jayemane 2012, 15)

The intensity of the video game as event or network rests not only in digital components and virtual gameworlds, but also in the particular ways in which they are instantiated - the intimate and cybernetic circuit between the human and the non-human. Here I mean cybernetics not in its loose discursive sense of 'something to do with computers' but literally as the material feedback of information and control between machines and organisms (Wiener 1961). Donna Haraway's work is particularly influential here. For Haraway, 'a cyborg is a cybernetic organism, a hybrid of machine and organism, a creature of social reality as well as a creature of fiction' (Haraway 1990, 191). This ambiguous status is bound up with the cyborg's descriptive and utopian possibilities for Haraway, and it is reflected in the diverse ways in which this figure has been discussed. For some it suggests that the posthuman is predicated on observations or warnings that the corporeal aspects of the human are, or will be, undergoing transformation. Others invoke the cyborg and the posthuman (following Latour, for example) to argue that humans and human societies have always been technosocial and hence never simply or exclusively 'human'. The question of what kind of relationship between the human and the technological is brought into being in video game play runs through this book, and the ramifications of conceptualizing everyday media experience as cybernetic or cyborgian will be addressed specifically in Chapter 5 ('Soft Worlds'). ${ }^{2}$

For now, I will give one example of how everyday play with digital media constitutes bodies and networks between bodies in new and distributed ways. Knowing my research interests in children and technology, a colleague told me how she often talked to her young granddaughter, who lives in the United States, via FaceTime on their iPads. The possibility of cheap and easy video telephony is in itself new, but skyping is becoming increasingly familiar and unremarkable. 
The portability of the mobile device and its wireless network connection, however, meant that the child could walk around with the iPad chatting to her grandmother on the move. It appeared that the fact that her grandmother's face could be seen on the device's screen made her more 'present', or even that the device (with its face and voice) stood in for, or fused, grandparent and communication device in a way more profound than the disembodied voice of the telephone. This was borne out when the child began a game of hide and seek with her parents, taking the iPad/grandmother with her to hide in a cupboard, whispering together in the dark.

\section{Ecology and ethos}

The notion that media are not discrete devices and channels, but are always already part of an ecology of other media, technologies, cultural patterns, technical practices and material substrates has proved productive in recent years (Fuller 2005, Parikka 2010, Taffel 2013), and draws on the ecological or environmental ideas of Marshall McLuhan (1964). This book takes a broadly 'media ecological' approach to everyday play with technology. On one level, an ecological perspective resists the tendency to see particular new technologies as having an 'impact' on the home, households, children and so on. Rather they must find their place and time within the existing technical and domestic environments. Children today are born into digitally augmented environments, there is no unmediated, non-technological environment on which devices impact. On another level, this natural-artificial ecology resonates with the images of tangled technocultural networks sketched out above. Moreover, it echoes the terminology of childhood research, particularly the educational and psychological studies that observe and interact with children in ordinary environments rather than setting up lab experiments (Avedon and SuttonSmith 1971).

\section{Assemblage}

A useful concept that can be loosely connected to all of these bodies of work is assemblage. T.L. Taylor explains how the term can help us to understand the complexity of technocultural phenomena, and contemporary videogame 
cultures in particular. She lists some of the heterogeneous bodies and agencies that 'make up the play moment':

Games, and their play, are constituted by the interrelations between (to name just a few) technological systems and software (including the imagined player embedded in them), the material world (including our bodies at the keyboard), the online space of the game (if any), game genre, and its histories, the social worlds that infuse the game and situate us outside of it, the emergent practices of communities, our interior lives, personal histories, and aesthetic experience, institutional structures that shape the game and our activity as players, legal structures, and indeed the broader culture around us with its conceptual frames and tropes. While looking at a game as it is presented as a boxed product may tell us something about the given structure of the artifact or its imagined player, understanding it as a living object - as a playful artifact - comes via an attention to the assemblage that constructs our actual games and play. (Taylor 2009, 332)

The events in and around Sandy Bay, on- and off-screen, cannot be described simply as children's play with a new toy or medium, nor would a detailed analysis of either the screen images or the structure of the game, its rules, spatial dimensions and so on. They were constituted by an assemblage, a set of tight loops through all of these entities, none of which could be said to be dominant, in control, or to have initiated the game event in the first place. This raises questions about the terminology and conceptual underpinnings of empirical research in media and culture. My initial term for my approach in the Lego Racers research was 'microethnography', referring to the small scale and short duration of the technocultural event under scrutiny, but also - more playfully - alluding to the micro-circuits and micro-worlds of the video game and the digital video technologies used to record them. The term 'ethnography', however, is not adequate for the description of this intimate technoculture. Literally the 'writing of people, ethnography, like its parent discipline anthropology (the study of humankind), always already has the human - bodies, language and society - as its object of study and focus of its attention.

\section{Ethology}

To examine how the game as software and computer, console, peripherals as hardware are part of, or participants in, children's lived media culture, I use a research approach that takes technologies (both hardware and software) and 
media images as its objects of study - or, more precisely, an approach that describes an event constituted by the circuits of agency, affect and play between human and technological participants. My term for it was initially temporary and playful: 'microethology'. 'Ethology' was suggested by my colleague Iain Grant. Since the nineteenth century, the term has primarily been applied to the study of animal behaviour. Gilles Deleuze (1992) gave it a different application through his reading of the philosophy of Spinoza to denote an ontology of bodies and affects, natural and artificial, in which no prior assumptions are made about the nature or primacy of these bodies. Ethology is an approach that defines

bodies, animals or humans by the affects they are capable of.... Ethology is first of all the study of the relations of speed and slowness, of the capacities for affecting and being affected that characterize each thing. For each thing these relations and capacities have an amplitude, thresholds (maximum and minimum) and variations or transformations that are peculiar to them. And they select, in the world or in Nature, that which corresponds to the thing; that is, they select what affects or is affected by the thing, what moves or is moved by it... an animal, a thing, is never separable from its relations with the world... The speed or slowness of metabolisms, perceptions, actions and reactions link together to constitute a particular individual in the world. (Deleuze 1992, pp. 627-628)

It should be noted that Deleuze's use of the term 'affect' is not limited to its more usual sense in the humanities and social sciences as the experiential and emotional aspects of human embodiment and subjectivity, although the latter would be included within it, or, rather, constituted as particular products of it. 'Bodies', for Deleuze (again following Spinoza), are not only human or animal bodies, but any material or immaterial entity in the world: microscopic particles, organs, machines, a body of literature and chemicals. As the quote indicates, bodies are defined in part by their affect on the bodies around and within them and by the affect these other bodies have on them. Ethology's concern, then, is with behaviour, and should make no a priori assumptions about the most significant bodies or agencies doing the behaving.

Gregory Bateson uses the term 'ethology' somewhat differently from Deleuze, but given that his use touches on anthropology, cybernetics and a broad ecological worldview, it is worth briefly summarizing here. For Bateson, the ethos of a social group refers to the lived, felt and affectual dimensions of culture. Though intangible, it helps to perpetuate and reproduce culture; in cybernetic terms, it is a corrective mechanism to the runaway transformative impulses of schismogenesis, where the latter is the social tensions of differences in power 
and hierarchy between individuals and groups (Bateson 1972, 126). Highmore describes the manifestations, the 'real concrete elements', of ethos in everyday communication and interaction:

[W]ays of greeting (hugs, handshakes, kisses, etc.); forms of perceiving (social recognition and misrecognition of class, caste, gender, sexuality and so on); affective intensity (the permission, or not, to be angry, the uses of humiliation, etc.); and so on. Ethos may well best be approached as something like a touching, or a feeling, but its polyphonic dimension, its complexity must be continually stressed. (Highmore 2009, 13)

Bateson's notion of ethos, and the anthropological, cybernetic and ecological fields in which he developed it, resonates with the ethological and ecological approaches in media studies and philosophy briefly noted earlier, as well as influencing his friend Edith Cobb's work on the ecology of children's imagination.

An ecological/ethological approach to contemporary children's culture, then, would resist a priori conceptual distinctions between individuals (the child, machines), social groups (families, households, classmates), technologies (hardware, software, toys, play equipment), physical environments (bedrooms, lounges, gardens, beaches) or symbolic environments (images and ideas from popular media, oral/practical traditions of play and games, etc.). So, in the Lego Racers event, cameras, domestic space, family relationships, screens, songs, movements, toys, and gestures, software, boredom, video editing and compositing, media/animated images and environments, are all in play. Material bodies (human, technological) are looped together with intangible circuits of information (light emitted from the screen, captured by human eyes and the camera, processed and transmitted by nerves, muscles and microprocessors), affects (competitive and collaborative performance and humour, the imperative to play itself) and imagination (itself distributed between the boys and looped out through the media imagery).

\section{Practical approaches}

The events I describe here are generally captured on video, though some were recorded mainly as written notes and some as photographic sequences edited to audiodocumentation. More often than not these microethologies are opportunistic and improvized. An interesting event seems to be unfolding so I pick up any 
documentary resources to hand. ${ }^{3}$ There is a long history of film and photography in anthropology, and digital media have made the visual documentation and dissemination of ethnographic studies inexpensive and accessible. In my own practice, I find that much of the descriptive or analytical work takes place in the editing of the audio-visual material. Video and audio recording captures an event in more depth than written notes - one finds unnoticed activities in the background, innocuous spoken phrases reveal themselves to be full of inadvertent insight, and complex connections and relationships appear as video and audio tracks are cut and placed on the editing software timeline. For example, I had made a video essay of the Lego Racers event (Giddings 2003) initially to explore the transduction of virtual to actual space. Through a straightforward montage, I placed moments from each in sequence - the cars set off from the start line/ the boys set off on their own race around the room; a car 'drowned' in the virtual sea/an actual Lego car was driven gently across the crayoned paper; and so on. Through the repetitions of viewing, logging, editing, rendering and reviewing, I began to question why the boys' actual movement of the cars over the plastic mountain was so graceful. So, though I had no intention of conducting a study of the transduction of virtual gravity, the process of media ethology proved a kind of extended and technically augmented deep description. Of course cameras can inhibit behaviour, though I have always found that children very quickly ignore their presence (and Alex and Jo are very used to being recorded anyway) perhaps from time to time remembering and leaping up to perform, mess about or - inevitably - push an eye or nose right into the lens.

I make more conventional notes too as soon as possible, to record aspects of the event that are not visible or audible, perhaps emotional states or connections to other events.

Though not all these studies are of my children, or of my children alone, being a parent ethologist has a number of advantages. It allows this microethological attention to the momentary and the small scale within the context of what is in effect a life-long longitudinal study (see also Sefton-Green 2004, Briggs 2006).

\section{Aesthetics, kinaesthetics and software ethology}

What do we need to add, to factor in, to incorporate virtual media and video game culture into microethological description? Images, characters and narratives flow from the more expansive landscapes of children's media 
culture into gameworlds, both virtual and actual. The aesthetics of cartoons, action, romance and nonsense are all agents in play (see Chapter 4, 'Media Worlds'). Both virtual and actual gameworlds are kinetic: video games are in effect interactive animation, and actual play is nothing if not embodied. From swinging to fighting, skipping to drawing, there is a technics and a kinaesthetics of physical play (Chapter 6, 'Playgrounds'). Finally, whereas the bodies of technocultural and sociotechnical theories sketched out earlier all emphasize the agency of technologies and other non-human objects, the software worlds of computer games have a particularly active mode of being. They are dynamic, semi-autonomous environments that change over time, and are populated with software robots and artificial intelligences. The ecology of these virtual worlds will be discussed in Chapter 5, but it is worth mentioning it now as it highlights the conceptual and methodological challenges of describing the virtual-actual worlds of contemporary life. As Jussi Parikka asks,

What defines software but its capacities, its affects, its relationality? Software is not immaterial but a body of code being executed, existing through that temporal unfolding in technological and other milieus that support (or afford) its existence. How different would software appear if we approached it as an ecological object that is capable of entering into specific relations (that are not only technical) and whose capacities for affect are due to certain internal and external milieus? (Parikka 2010, 165-166)

Any qualitative research method, of course, has its strengths and limitations; a very small scale and improvized microethology, such as the one presented earlier, can make only heavily qualified claims to identify broad cultural trends or patterns of media consumption and play. However, it is important to note that whilst studies with much larger samples and extended longitudinal reach might give a statistical sense of scale, access, distribution, discourse and attitude, they are unlikely to be able to say much about emergent phenomena, practices and behaviours. By necessity, any study of the detail and texture of any specific cultural or media practice as experienced by individuals or small groups is small scale. Also, any identifiable deviation from and innovation in established practices of media consumption are significant, but may initially be evident only at the local level of actual engagement. At the very least, a microethological approach can identify emergent behaviours that can then be looked for, and tested, in largerscale research. I am concerned throughout with the intimate and the complex, not with the general or the scientific, but I hope that these descriptions are rich 
enough to offer models or patterns of relationships and behaviours that can be applied or compared with other children, other assemblages.

My reading of others' research on children's games and play, pre- and postdigital, is presumptuous in its identification and selection of descriptive material that most closely fits my own approach - the details, chance encounters and phantasmagorical moments, but not necessarily the conceptual or culturalpolitical framing of the writings from which they are plundered. Though perhaps modest in its findings, an ethological approach offers a nuanced theorization of the relationships between the virtual and the actual, the technological and the social, and media images and imaginations in everyday play. Children's play, a domestic environment, and a computer game are reciprocally transformed, the everyday lives of children and adults are permeated (as we'll see in the next chapter) by colourless energy, by entities that are hybrids of computer code and symbolic figures and by hyper-real gravity. It is no accident that I have quoted the more exploratory, descriptive passages from these case studies as, I would argue, they demonstrate the necessity of description in teasing out and attending to the new and hybrid as well as the established and the familiar.

\section{Notes}

1 See Giddings 2006 for a brief sketch of their workings in relation to videogame culture.

2 There are other rich methodological and conceptual approaches to the embodied relationships between computer technology and people within Human-Computer Interaction (HCI), for instance. See Paul Dourish's study of embodied interaction and technomethodology (Dourish 2004), Lucy Suchman's anthropology of humanmachine interactions (Suchman 2007) and Mark Hansen's phenomenological exploration of virtuality (Hansen 2006).

3 I have also conducted more organized and experimental video-ethological studies of gameplay, usually in collusion with members of the Play Research Group, and Helen W. Kennedy in particular (Giddings and Kennedy 2008, 2010). 


\section{Media Worlds}

I use the term 'worldly' to indicate the of-the-world and participatory nature of contemporary children [... This is] the childhood reflected in the complex problem-solving, intertextuality and non-linear pathways of the Rugrats computer game. These texts, and others like them, situate contemporary children in global flows of consumption, identity and information in ways unheard of in earlier generations and contribute to the construction of new discourses around childhood. Where once children grew into literate habitus within a relatively confined, predictable and supervised field of information and discourses, this is no longer the case. (Carrington 2005, 22)

Video games and other popular virtual media emerge from and intersect with the established forms and channels of children's culture in a complicated media ecosystem. They are in themselves popular entertainment media: animated characters and environments in vivid colours, sounds and music, played out on TV screens and monitors. The video game is a commercial media object, a commodity often fully part of a wider media symbolic universe - a game version of a film or television programme or even a book. It might emerge itself from the cross-media commercial strategy of contemporary transmedia systems such as Pokémon and Lego. Even video games with their own self-contained fictional world (the Animal Crossing or Grand Theft Auto series, for example) are fully part of the everyday media ecology, drawing semiotic nutrients from it, competing for the sunlight of players' attention with other games, media objects and devices, sending shoots out through the Internet media of walk-through and cheats sites and flowering other forms and moments of play.

They are also, of course, games, bringing the ludic structures and long histories of board, card and playground games to electronic and digital 
media, challenging the dominance of narrative as a fundamental organizing principle or form whilst also effecting a confluence with other streams of children's media culture such as game shows, quizzes, competitions. Games in themselves, whether digital or not, are a medium. Marshall McLuhan devotes a chapter to them in Understanding Media: The Extensions of Man. Thinking mainly of sporting events, he regards games as utopian performances, enacting a microcosmic model of a national cultural identity, but entwined with and transformed by television (McLuhan 1964, 254-266). Video game consoles, particularly handheld devices, might be shelved with other toys in the shops, and some virtual media objects, such as Tamagotchi, are a hybrid of toy and computational media.

The encroachment of popular media (often commercial) from comics to radio, cinema to television, in children's culture, their hold on children's attention, their structuring of daily lives and the messages they may channel have all contributed to a widespread sense of an incremental transformation of childhood. The precise nature of these perceived changes varies tremendously, but they tend to assume the worst. The dating or periodization of these changes is similarly varied yet often coincides with any particular commentator's own life history - that is the most serious decline is perceived to have occurred since that individual's own childhood. For generations now it appears that children have played traditional games with friends outdoors, then grown up to a world in which their children seem to be fixed indoors, in thrall to electronic media, solitary and inactive. Writing in the late 1970s, Iona Opie noted several generations of adults observing her research on playgrounds and remarking that games were more structured and sophisticated when they were children (Opie 1983). This is not to argue that there have been no changes to children's culture and everyday lives - and I will briefly set out some of these changes below - it is instead again a call for a closer attention to what children are doing with their media and with their play, and a call for a trust in play itself in the face of, and as a mode of living with, change. Another deep source of anxiety concerns the representational operations of children's media, the messages that have been sent from the Penny Dreadful and the first-person shooter. In Chapter 2, I questioned the dominant critical assumptions that the significance of media lies in their representational and ideological operations, so this chapter will address the question of what, if anything, games as media might communicate. 
Predictions and diagnoses of decline or catastrophe today are bound up in the new characteristics and operations of digital and communications technologies and networks, as well as the commercial targeting of children as consumers. The immersive virtual gameworlds, social media networks and commercial franchised transmedia systems of the past ten or twenty years all work on childhood, imagination, media influence and nature itself. There are smaller, but still significant, questions to be asked about the nature of children's media when narrative forms are displaced or augmented with ludic forms. Through ethnographic and ethological case studies, this chapter will track some of the ways in which media worlds, rather than being necessarily bounded, selfcontained playgrounds for (or shackles on) imagination, are often used instead as material for extensions of games and play, generally referred to as meta-games.

Finally, an understanding of digital popular media must describe and theorize the collusion of computer technology and children's media (and this issue will be explored further in the next chapter).

\section{Media histories of decline}

Historical studies of play present a more complicated picture of the mediatization of children's oral and game cultures than the narratives of homogenization and extinction. Howard P. Chudacoff's useful history of children's play in the United States echoes the common view that there was a golden age of play in the first half of the twentieth century - a historical moment begun with the removal of children from the workforce and the expansion of compulsory education and ending with the advent of television and its 'appropriation of children's culture' in the mid 1950s. Yet he also traces adult intervention in play, and attempts to control play, back to the midnineteenth century. Here, then, is a much longer trajectory of directing play into the worthy channels of education and learning, moving it into purposebuilt playgrounds and parks and away from the dangers of rivers, waste grounds and woodland. The intense urbanization saw the concentration of children in cities, adding to this shift in the environments of play:

Play environments shifted as the urban-built environment offered new sites and material. Nature still gave youngsters sites for 'roaming, and domestic spaces still could be appropriated for games and fantasies. But now, paved streets, better 
lighting, telephone poles, fire hydrants, and fences increased hazards but also provided opportunities for playful inventiveness. (Chudacoff 2011, 105)

Alongside, this period saw the rise of children's consumer culture for middleclass children, shifting play into the home with a new commercial toy industry (Chudacoff 2011, 105). Many of these toys and games facilitated solitary as well as social play. The 'sheltered-child model' has a longer history than the last couple of decades of 'stranger danger' and domestic children's digital culture.

Whilst the move to domestic or structured playspaces and the increasing commercialization of play have a longer history than is often recognized, there are clearly subsequent moments at which play and children's culture underwent significant transformation. Television from the mid 1950s not only offered children a media alternative to board and card games or books, but its commercial imperatives (in the United States at least) also drove an intensive targeting of children as the consumers of toys and merchandizing. Indeed, from The Mickey Mouse Club in the 1950s to the new economic models of 'limited' animation and toy tie-ins of the 1980s (Kline 1993), children's play culture was increasingly intertwined with media and consumer culture. The Disney Corporation was at the heart of conflicting attitudes to children's media culture in the twentieth century. The integrated marketing of characters and environments across films and other media, licensed products and merchandizing and theme park rides and displays prefigured the transmedia universes of contemporary commercial children's culture (Forgacs 1992). The imagineering (to use Disney's own phrase) of physical and semiotic worlds for some critics was so sophisticated and all-encompassing in their manipulation of pleasure that it marked a total commodification of not only play but children's imaginative and fantasy lives:

we are faced with a growing commercial sphere that profoundly limits the vocabulary and imagery available to youth and others [...] What we do not need is a global culture industry in which Disney imagineers and executives turn children's desires and dreams into fodder for advertisers and corporatecontrolled media. (Giroux and Pollock 2010, 15)

As a critique of the expansive cultural economic reach of corporations like Disney this is laudable, but the claims that 'our most intimate perceptions and desires' are being conditioned (Giroux and Pollock 2010,2) are founded on sweeping assumptions about the nature of children's everyday engagement with media. Thus, children are increasingly 
left on their own to navigate the virtual and visual worlds created by U.S. media corporations. Even if many of these kids do not end up developing obesity, attention deficit disorder, anxiety, and depression, to name just a few long-term effects of media consumption, they still lack the adequate nurturing, guidance, and support they need to feel secure and happy entering their adult lives. (Giroux and Pollock 2010, xvi)

Kieran, aged three years and seven months, loved to wear his grandmother's high heel shoes as he watched 101 Dalmatians. The shoes were always kept next to the television. His mother stated,

He re-enacts it. That's why we've got my mum's shoes over there. [To child] Cruella Deville, aren't you? You're Cruella Deville, aren't you? [To interviewer] And he re-enacts everything that he watches in 101 and 102 all the time... and then we'll re-enact it but we'll re-enact it for about two weeks. Honestly, if he's really found it funny or something he can talk and talk and talk and talk. And he talks about the same thing over and over again. (Marsh 2005, 41)

And yet, studies of children's play with media resources, including Disney, such as this indicate that children rarely, if ever, fully inhabit and act out a prescribed media world, whether that be a book, film or TV programme. The passage of media images, narratives, worlds and action however well coordinated and systematized can never be simply adapted or translated from their particular 'platform' to their performance in play. They are transformed in the negotiations of collective play, or in performances and repetitions such as those of Kieran in the earlier example. The simple fact of transduction from one kind of gameworld (a Disney film or transmedia storyworld) to another (a group of children in a specific environment) necessitates a more or less surreal actualization. As Gilles Brougère notes in a discussion of the circuits through the mediated and lived worlds of Power Rangers,

even when the children try their hardest to match their own play behaviour to that modeled for them by television [...] they are forced by their need for cooperation to make all kinds of compromises, such as bargaining for who takes the negative roles, deciding how they can adapt their unique 'power' feelings to the scenario, devising costumes, weapons, gestures, and sequences. What they reproduce is a playful theatric adaptation. There is no tabula rasa. The point is, no matter what the cultural stimuli might be (toys or television shows), they have to be mediated by children's fantasy in order to be accepted, and adjusted to their play norms and social competence in order to be assimilated into the active theatric play forms of childhood. (Brougère, in Sutton-Smith 1997, 154) 
At the very least the constraints and affordances of the physical environment (available toys, space, play equipment, weather, interruptions of other children, adults, the temporal boundaries of school playtime, children's bodies and their capabilities - see Chapter 6, 'Playgrounds') and of the imaginative environment (compliance of fellow players, depth of understanding or memory of the storyworld) mix up, distort, transduce the characters, narrative, magic powers, technologies and so on into a dream-like version. Shanly Dixon and Sandra Weber's account of a group of six-year-old boys and girls playing a game of 'Snow White' illustrates this. To capture the construction of this collective gameworld, and its flow of embodied and symbolic behaviour through and beyond the narrative template provided by Disney, I'll quote it at length. The game began with a cry of 'let's play Snow White' and intense arguments over who would be Snow White herself:

One girl has dark hair and soon convinces people she should have the role because she looks like Snow White, although another girl claims that the fact she has a Snow White lunch box gives her an edge. An older boy suddenly announces that he wants to be the wicked Queen because he has a cape he could use as a costume. No one seems to want to be the prince. The children begin enacting the story, stopping and restarting, giving each other directions $[\ldots]$ and improvising many touches that bear little resemblance to the movie. The game stops and then starts up again as one child tickles another, or someone calls attention to the mushrooms growing on the damp ground. Hilarity breaks out when one boy decides he is a deer who discovers Snow White asleep in the woods and tries to wake her by licking her [... Snow White is called home by her mother - and another girl suggests her Barbie doll could take the part of Snow White. She proceeds to use her Barbie as a puppet, acting as ventriloquist for 'Snow White Barbie'. Another child goes along with this, but two others just sort of parallel play a different version of Snow White. The play keeps falling apart (to my adult observer eyes at any rate) changing directions, as a couple of boys engage in rough and tumble play, one child bursts into tears saying 'I don't WANT to be Dopey', and so forth. And then the dwarfs start singing a popular song, 'I'm too sexy for my shirt' and dancing around, while two girls decide the piece of cardboard they were using as the Mirror Mirror on the Wall is not very good, and announce they are going to make a 'real one'. (Constructed from Weber's fieldnotes, August 1992). (Dixon and Weber 2011, 483-484)

Dixon and Weber suggest that far from being programmed by the Disney Corporation, this imaginative play realized (surrealized perhaps) a 'cumulative popular cultural text', not a slavish reconstruction of the film, but 'an evolving 
script' that swept up and filtered memories and materials from toys, lunch boxes, non-Disney storybook versions and so on.

Through the children's active play, negotiations, and improvisations, the text was adapted, ignored, disrupted, and reconstructed. There was a fluidity to the play despite a series of abrupt halts and changes of directions as the children moved in and out of the various roles that they had assumed. (Dixon and Weber 2011, 484)

The Snow White game was observed in 1992, however, and the subsequent twenty years or so have seen further transformation of children's media culture. Disney's cross-media stories and characters could now be seen to prefigure the convergence or dissolution of discrete media forms into the synthetic worlds of video games and the global information networks of Internet media. Over the decades since their arrival in living rooms, video games have not only drawn on media franchises and genres, and fed back into them, but also spawned new hybrids of games and programmes, merchandise, toys, websites and 'advergaming'. This hybridity is hypercharged by digitization and the networked distribution of all popular media along with the emergent participatory and fledgeling co-creative practices of the web to form new media ecologies in which images and narratives are functions of the ludic mechanics of navigation and accumulation. Films, TV and toys have become subsumed into the ludic forms of games, social media and networks, from the carefully moderated virtual worlds of HabboHotel and Disney's Club Penguin to services such as Facebook (officially populated by an older age group, but which attract young children too): networks that are characterized by an aggressively commercial motive. Neopets, for instance, is an online world, a vivarium of 'virtual pets', but one dominated by what the company calls 'immersive advertising',

defined more specifically as 'creative programs that integrate the advertisers' commercial products, services, brands and names into existing or customized activities and scenarios within the site, thereby making the product an important part of the activity or game.' (Wasko 2010, 118)

The site is a mix of product placement and advergames as well as banner ads and commercial sequences. Janet Wasko's analysis of the site echoes earlier critiques of the Pokémon universe of acquisition as a kindergarten for consumption (see Lister et al. 2009, 292-294):

The Neopets site is not just about adopting and caring for a pet - it can easily be interpreted as a training ground for capitalist consumer culture. While other 
values or ideas are represented on the site, themes related to materialism and consumption are particularly overt. Neopia is organized as a capitalist society, as is apparent, for example, in its bank and stock market, as well as the game 'Plushie Tycoon' where one can become a successful manufacturing tycoon. There are lots of shops [...] players are encouraged to acquire (buy) items and sell them for Neopoints [... ] A recent addition is the NeoCash Mall where items are available for 'real world' money. Customized clothes and decorations can also be purchased through Neocash cards, which can be purchased through PayPal or at participating stores. (Wasko 2010, 119-120)

Privatized gameworlds like Neopets 'naturalize the commercial process that is at the core of advanced capitalism' (Wasko 2010, 127).

However, these critiques of entertainment supersystems and transmedia franchises read off their effects and uses - the everyday cultures, it is assumed, they generate - from the imagery, investment and intentions of the corporations that produce them. There is a grudging acceptance that the world of Disney has its pleasures, but this pleasure is utterly passive and no genuine play - semiotic or material - is possible. Janet Wasko asserts that Disney texts in particular are closed off to play and imagination:

there is little room for active or alternative readings of texts, like Disney's, which are carefully coded and controlled, and not polysemic and open. (Wasko, in Giroux and Pollock 2010, 11)

On one important level these worlds function as designed - they are successful because they capture children's imagination and instigate a circuit of attention and a flow of money from advertisers and in-world purchases. Yet these critics offer no evidence for their claims of semiotic and playful closure, or for the notion that children's imaginations are colonized or rewired by the ruthless logic of consumer capitalism. On the contrary, empirical and ethnographic research on everyday transmedia cultures suggests a more vibrant, participatory and creative engagement with media play.

Again Pokémon is key here. A Japanese media world designed as transmedia system from the start, it revolved around the Nintendo GameBoy video games and collectable playing cards, and this dynamic of collecting both actual cards and virtual creatures ('gotta catch 'em all') assumed its young players would read, watch, collect and play across comics, toys, television, cinema and merchandizing. Significantly, game mechanics are central and generative to the transmedia system rather than being mere merchandizing spin-offs from the narrative media of television and cinema. Indeed, as Julian Sefton-Green 
observed in his son's obsession with Pokémon, enthusiasts needed to embrace transmediality to fully engage with, or even to progress through the games. Cheats and hints must be sifted from magazines or online forums and walkthroughs, or elicited from other players (Sefton-Green 2004). Connecting GameBoys with a cable allows the trade of rare and desirable monsters. As a media storyworld, engagement with a Pokémon game is just as 'imaginative' as other storyworlds, but it also investigative: a process of research, learning, trial and error, puzzle solving and deduction. They are built for and anticipate player/ consumer activity, and are only fully realized within it. This activity includes not only purchasing the elements, but collecting them - which entails swapping with friends, developing specialist knowledge of rare items, of shops that may have new stock, and for the aficianado, specialist events and forums:

new convergent media such as Pokémon require a reconfigured conceptual apparatus that takes productive and creative activity at the 'consumer' level as a given rather than as an addendum or an exception. (Ito 2011, 494)

As Mizuko Ito concludes from her ethnographic research on Japanese children and transmedia systems (she prefers the Japanese vernacular term 'media mix'), along with convergence, these new cultures are characterized by

authoring through personalization and remix, and hypersociality as a genre of social participation [defining] a new media ecology keyed to a more activist mobilization of the imagination in the everyday life of young people. (Ito 2011, 492)

Whilst the thousands of available cards, and the manipulation of rarity (tried and tested strategy from the era of collectable cards in bubblegum and boxes of tea in the mid twentieth century), are conducive to sustained consumption in particular (and perhaps a general orientation towards the accumulative pleasure of consumer capitalism more broadly), this array of characters and monsters also support players as:

a massive collectively shared imagination as source material for producing local identities and performances. The practices of talking about and exchanging cards are an example of hypersociality as a genre of participation: the merging of local social negotiations with the exchange of media-based knowledge and signifiers. (Ito 2011, 500)

Video games and other playful digital media, then, mark a significant transformation of children's culture at the beginning of the twenty-first century. Causal relationships between these new media and perceived new patterns of 
consumer-driven, solitary, even morbid, play are not so easily established. The video game's arrival in living rooms (at the end of the 1970s) coincided with an increased awareness of, or perception of, dangers of child abduction and abuse, a continued rise of traffic levels that rules out street play in large parts of the urban centres, a tendency in middle-class families in particular to structure children's lives around developmental activities (music lessons, organized sport, etc.) for social status or to improve educational and career prospects. Parental attitudes to digital technology are often deeply ambivalent: computers are seen as essential for education, yet at best these educational aims are constantly diverted by the pleasures of gameplay, whilst at worst they threaten to inculcate isolation, screen fixation and to allow in new predatory threats from cyberspace. Computer games and interactive toys exacerbate concerns that arose with broadcast media that children's imaginations wouldn't be given free rein but would be channelled by, or simply filled by, the spectacular images and dramas. The generic character of pre-electronic toys (dolls, cars, rattles, building blocks), it is often asserted, helped small children to be the 'creators and controllers of their play', whereas these individualized and open-ended opportunities for play are greatly hampered by programmed responses and scripts', limiting not only play, but also 'the child's imagination and development' (Levin and Rosenquist 2001, quoted by Plowman 2007, 210-211).

\section{From gameworlds to playworlds: Material for other play}

As we have seen, critical work on children's media culture is generally concerned with the representational operations of programmes, cartoons, toys, and films and the messages conveyed to impressionable minds. Specific questions of identity (Disney princesses and gender for example) may be addressed, or broader concerns about the induction of children into the adult worlds of consumer capitalism. These representational processes persist, or are accentuated, it is assumed, in the new structures of interactive games and toys. However, the ways in which these 'messages' are actually lived and played are often assumed and not examined. Put simply, how can we know what meanings children make of their media? Or even if 'meaning' is the most relevant mode of engagement with media in this context? Children play with their media TV programmes and films as well as board games, videogames and toys. Play is rarely a process of unimpeded flow of 'preferred' meanings; it synthesizes 
and condenses, it upturns the world as well as mirroring it. Secondly, video game culture brings the technological nature of media to our attention. The materiality and specificity of computer/entertainment hardware and software is a key concern of this book and must be central to any assessment or description of the processes of signification and use. Again the medium is the message/ massage: whatever the symbolic or ideological content of children's media, the modes of interaction, play, the arrangement of bodies as well as images, of performance as well as narrative are at least as important. Children use digital media - and vice versa.

I'll give further two examples of play events that are formed in large part by popular media and that, along with a playful manipulation of their visual and thematic material (like Snow White above), are characterized by a transduction of physical behaviour: the embodied and kinaesthetic pleasures of singing, dancing, drawing and role-playing. Firstly, through the historically well-established patterns of girls' singing and dancing games, and, secondly, through the contemporary transmedia system of Pokémon through which playful semiotic and material engagement in gameworlds including the virtual is assumed and scaffolded.

\section{Singing games and the media}

Singing, skipping and clapping games are among the most thoroughly researched and documented aspects of children's play. Studied by folklorists and musicologists, and more recently by researchers on media and education (Opie and Opie 1985, Marsh 2008, Willett 2011, Willett et al. 2013), their transmission across time (some songs can be traced back over a century) and space (by individual playground, city, region and internationally) can be followed, and their shifting form and language analyzed in detail. As a living oral (and behavioural/ gestural) culture, they contain archaic rhythms and references and accrete new material as they are taught and performed. Current pop songs, dance moves and jokes are woven into persistent traces of older songs from music hall, cinema and radio, nursery rhymes and fairy tales, as this Hollywood-inspired song recorded by Iona Opie in various versions across the 1950s indicates:

I'm Shirley Temple, the girl with curly hair

I've got two dimples and my skirts up there

I'm not able to do the Betty Grable 
I'm Shirly Temple, the girl with curly hair.

I've got a leg like nobody's business

I've got a figure like Marilyn Monroe

I've got hair like Ginger Rogers

And a face like I don't know

Oh Salome, Salome

You should see Salome

Hands up there, skirts up there

You should see Salome

Swing it, swing it

You should see her swing it

Hands up there, skirts up there

You should see her swing it

The Opies recorded numerous other references to Shirley Temple in such songs, dating back to the 1920s. In some the star was interchangeable with Charlie Chaplin as their names provided the same rhythm of syllables. Like other forms of play (and dreams), singing and skipping games sometimes play on the boundaries of taboo material, particularly violence and sex. Opie notes that this iteration of Shirley Temple was seen as 'a bit naughty'.

Outside the circle the boys take up vantage points, clambering on to windowsills or hanging on drainpipes like monkeys. 'It's because when we lift our skirts up the boys can see our knickers', explained a nine-year-old. (Opie and Opie 1985, 419)

In a recent study of a London primary school's playtime culture, Rebekah Willet watched the performances and play of a group of girls aged between five and seven years. This included performances of the song 'Breaking Free' from High School Musical with the girls " "flying" around the playground in a "follow the leader" formation (repeating the lyrics "We're soaring, flying"'), whilst others lined up their My Little Pet Shop toys on an improvized stage and, hiding, pretended that the toys themselves are singing and performing. The girls took their performance of JLS's 'Everybody in Love' and 'embedded [it] in a game of kiss-chase. According to the girls, they were singing this "love song" (or more accurately, singing the line "if you're in love, put your hands up") in order to taunt the boys in their kiss-chase game' (Willett 2011, 347).

In one sense, media images, songs, gestures, repertoires and themes become just more material to be stitched into play, and their performance seems to have little to do with identification as a character or as a consumer. Rather, these 
media resources have more to do with their rhythmic potential as the framing of skipping or dance sequences. As Willett notes, the girls select catchy chorus lines that are easy to learn and that 'combine a variety of actions and modes of engagement' (Willett 2011, 349).

These children are 'meme engineers ... active agents in the process of cultural change as they select, reject, adapt, modify - in short "engineer" - their musical narratives.' (Barrett 2003, cited in Willett 2011, 342)

They are also material for the ritual and collusional processes of initiating, sustaining and renewing social groups and friendships through play - in the metroptic sense of communication set out earlier. Dances and lyrics can be taught to new friends in processes of enculturation, signalling the group's identity to the rest of the playground:

it is not only a visual display of their friendship (doing things together), the playground can hear the sign (particularly as they sing the songs in a high nasal tone), and it is a sign that they share the same interests and resources. (Willett 2011,350)

\section{Transmedia systems: Drawing Pokémon}

As a transmedia system, Pokémon is designed to open up numerous and varied platforms for play with its characters and world. Our house was for years a monument to its success in this regard. Bedroom walls were covered with posters detailing and categorizing the hundreds of creatures, each name known by heart. Every birthday and Christmas brought a fresh deluge of plastic and cuddly toys, T shirts, books, games and trading cards - endless piles of cards to be stacked, sorted, filed, forgotten and refound. The detritus settled in corners and drawers, kicked around and un-noticed, until the demands of a game picked an object up and slotted it back into play. The boys talked about and enacted energies, evolutions and powers constantly, their parents learning enough about this artificial ecosphere to answer basic questions, particularly the frequent 'What's your favourite Pokémon?'

Alex drew Pokémon and Pokémon-like creatures incessantly. The attraction of drawing for young children is not the completion and display of accomplished pictures. It is a process of imaginative play in itself, an unfolding of imagined creatures and action into the material world, stories, combat or characteristics 
are distributed across the mind and the paper, often akin to a form of animation rather than a freeze-frame or map. The duration of the drawing is itself the game. Watching her son Douglas drawing vibrant battle scenes, Sylvia Feinburg's noted that in this dramatic play

Specific subject matter was less a common denominator than the general orientation and disposition of the activity, i.e. the process, the purposes it seemed to serve, the inclusion of such elements as confrontation, danger, motion, power, and co-operative effort. (Feinburg 1976, 589)

The environment - land, sea, architectural complexes - would be set out, then the men and equipment and then 'the encounter would begin'. The child would

enter the combat as a highly active participant, moving his body ferociously to simulate the destruction that might be imposed upon a single human being or piece of equipment, pounding with his fists ferociously ... sometimes two or more crayons in each hand simultaneously ... rocking and rolling, and emitting diverse, relevant accompanying sounds. (Feinburg 1976, 589-590)

Alex is drawing quietly, with rapt attention and precise marks. He holds pens and crayons very close to the tip so that fingers, mark-making point and paper all touch. I try to prompt him: 'what are you drawing?'

\section{A: A Pokémon}

S: What kind of Pokémon is that?

A: Just a Pokémon.

Either he is so engrossed in the creation of the creature that he is simply deflecting my question, or there is a creation happening here that is not to do with the specificity of the media text and characters. I suspect the latter. Pokémon is so integral to the ecology of the boys' material and imaginary lives at this point that 'Pokémon' could simply function as the rationale for beginning to draw, a spell to invoke the gameworld into existence on the paper. The imaginary world, if that's not putting it too strongly at this point, is vocalized through a loose repertoire of sing-song words and phrases.

A (quietly): Who's going to win the world? World champion ... Whoa, it's metal things.

At this point the drawing mode shifts up a gear. The 'metal things' appear as Alex has picked up a silver wax crayon. He draws more quickly, a spiralling scribble over an area of already intense overlaid scribbles of different colours. 
A: Look at these metal things. One of them's destroying... (he traces a silver line carefully along a figure drawn earlier across the top of the paper - something like a worm or spine).

A: They're giving their attack to another one (fierce scribbling now). You know what, this one just went skrwerch! and scribbled everything! Even its attacks!

A: Da-da-da-da

The plastic arts are always a coming together of ideas and aesthetic conventions with the material possibilities of the media: marks, colour, pattern, shape and so on. Felt-tipped pens set out clean, definite lines and edges, whilst wax crayons allow - and hence suggest - overlaying meshes of scribbles. The moment and action of drawing and the marks on the paper become the action itself: the actualization of the creatures' magical attacks like ectoplasm in a Victorian séance photograph (Figure 4.1). Scribbles are at one moment the representation of an attack (attacks depicted in the Pokémon games and comics as graphic lines of power, force and affect), and with a skrwerch, a crayon counter-attack - a meta-attack.
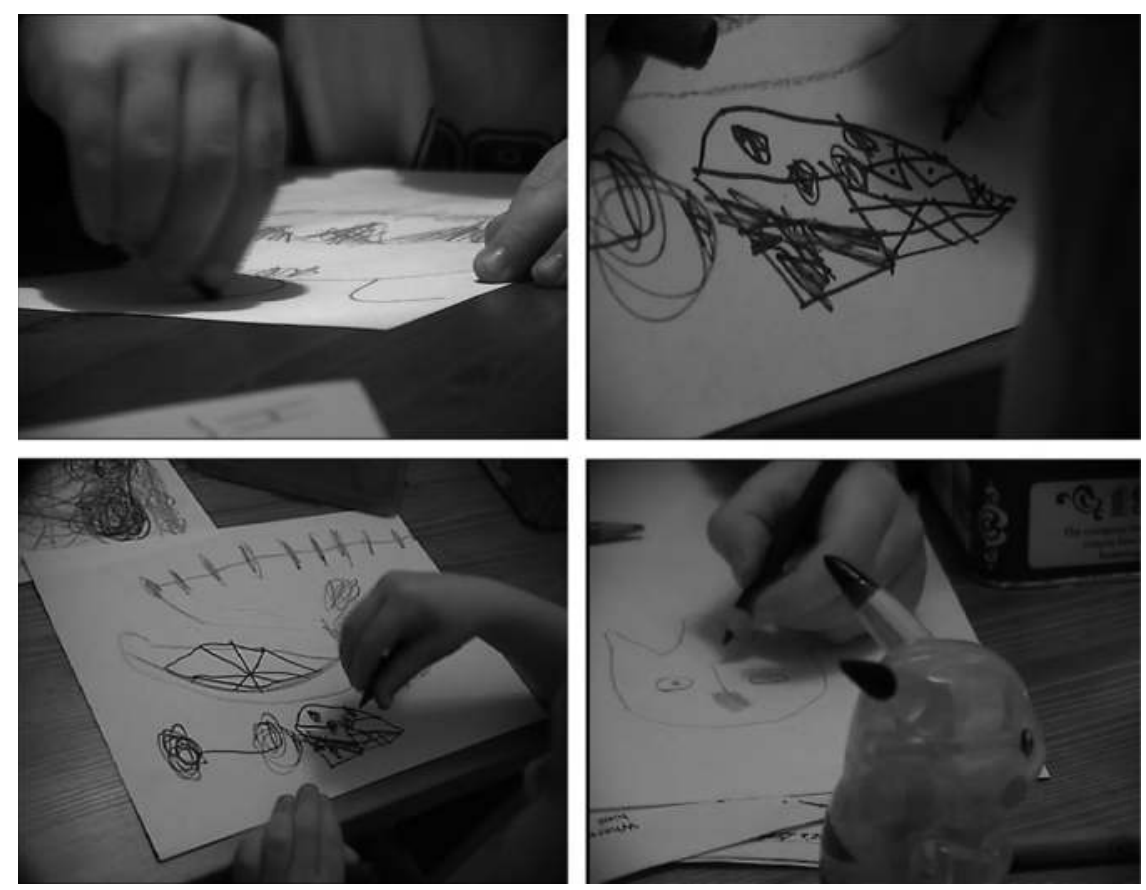

Figure 4.1 Drawing Pokémon 
He turns the paper over, and - calm again - with a black pen traces large letterlike shapes. Capital W, M, a mirrored N. Then a jagged little figure with a dot in each spike on its body.

A (concentrating on the paper, but addressing me now, albeit absent-mindedly): Do you know what has two eyes? These are just lines.

He is conjuring up a fish-like creature, a large body - segmented, with a dot carefully placed in each segment.

A (pointing at a line he has just drawn): That is not its mouth - that's a line. Look at that whale one.

$[\ldots]$

A: If you see very carefully... Daddy, do you think this is Colourless or Water? (these, I know, are types of Pokémon)

S: Errr ... Water?

A: Colourless!

A: This one has a bandage on its tail (he scribbles over the tail with his silver crayon). That's the bandage!

A (talking to his drawing): You should do attacks what I do, what I say you should do. That Pokémon, that's what they're allowed to do. They can do it their-selves.

That night Alex read his picture to me in bed as a bedtime story. I didn't video record this, and don't recall the details, but readings of this sort were generally a series of events and moments of action and combat traced with his finger across the paper as if pointing to comic book frames. The drawing provided a couple of characters, but a much broader and more fully populated world would have been improvized in the moment. He then stuck the picture to the wall with blu-tack. Jo came in and I read them both a story from a Pokémon annual - Jo correcting my pronunciation of the creatures' names.

The next day:

A: (pointing at an intensely scribbled part of his drawing) if you look very carefully you can see his eye - look!

J: It's an orange dot!

A: Yeah.

Jo is drawing too, off camera, but the microphone picks up his chatting and rummaging through the box of pens and crayons. Alex flips his paper over.

A: (indistinct) ... use the other side of the paper... I bet it's another Pokémon what it evolves into. 
This 'evolution' is an important aspect of the game and attendant stories as each creature is located within a short chain of transformation that is, strictly speaking, closer to the metamorphosis of insects and amphibians than the evolution of a species. For instance, every Pikachu evolves from a Pichu and into a Raichu.

The two stages of the transformation are being pictured on either sides of the paper. Alex draws a bold zigzag line across the page, then a straight line lengthways along the middle of the zigzag pattern.

A: Look, his teeth has got a line across it.

He flips the paper again and counts the angles in his zigzags.

A: This one has four teeth, but this one (he flips the paper and points as he counts) has $1,2,3,4,5,6,7$ teeth!

[there is more discussion of teeth and the number of teeth he and I have]

Jo is in shot now, drawing what looks to me like a ghost. A plastic Pikachu toy looks on, smiling enigmatically as Jo sings quietly to himself.

A (off camera): And this is what it evolves into. It has teeth. All Pokémon have teeth don't they.

J: Not all Pokémon.

A: Pikachu can bite, can't he!

J (pointing at his drawing): Look at that! It's a jellyfish Pokémon!

A: Wow, that's a cool one.

J: It's Tentacool.

A: Tentacool!

J: Remember Tentacool?

A: Oh, yeah.

The talk for the next couple of minutes ranges over a number of Pokémon-related concerns, returning periodically to Tentacool and the nature of tentacles.

A (referring to his drawing): It has bigger eyes too, than what it evolves into. It looks like a Tyrannosaurus Rex.

A: Yeah, but it has tentacles. On its head it has tentacles!

A: ... is that really what tentacles look like?

In this sequence, the drawing is not driven by the dramatic generation of particular Pokémon and their world, but rather by an intense exploration of physical attributes - teeth, then tentacles. The tentacles are closer mimetically to 
the images of Pokémon in the boys' games and comics, whereas the huge teeth seem to emerge from Alex's own immediate concerns, as - though technically monsters - Pokémon tend to be cute in appearance and not particularly fearsome. The drawing, then, is perhaps a touching or tracing of the contours and features of these compelling creatures, a summoning. Like Douglas' battles, bringing together 'a visible, concrete reality with a symbolically constructed reality' (Feinburg 1976, 591).

A little later the drawing begins to spin out into gestures beyond the page. Alex has drawn a fish-shaped creature.

A: It's a Powerfish! (not an actual Pokémon). Because it's got a... (he swirls his pencil around the fish's tail) ... because its tail spins around! So it swims!

J: Like this? (he flaps his hand) Up and down?

J: Or twirling around? (twirling his hand)

A: Twirling around!

$\mathrm{J}$ : Or up and down?

A: Twirling around.

A (sings): Twirling around, twirling around, twirling, twirling, twirling around.

The boys themselves are twirling now, the drawing is more intermittent, punctuated by gestures, actions and movement away from the table. Before long the table is abandoned and Alex bounces round the floor repeatedly demanding that we 'Guess what Pokémon I am!'

Jo is holding a small bird Pokémon toy up for the camera - Pidgey I think.

A: Guess what Pokémon I am!

A: I'm small ... and spiky. (He bounces up to me on all fours).

J: Primape!

Alex bounces off again, fists held up in a simian gesture.

A: Primape! Primape! (It is a feature of Pokémon that their vocalizations are only their own names or abbreviations of them. Pikachu says 'Pika! Pika!' for instance).

A: Primape does do this, doesn't he.

Jo joins in the jumping and Pokémon-guessing game.

J: Try this one: what is small and has a flower on top?

A: Don't know.

J: An Ivysaur.

A: Oh, yeah. 
Jo jumps on Alex and pins him to the floor, Primape and Ivysaur jumbled up in the rough and tumble, dissolving into an occasional shout or gesture. At some point it becomes apparent that Primape has been replaced by Pikachu and that the botanical Ivysaur must have evolved into its next stage: Bulbasaur.

(Fidelity to the Pokémon universe is lost in this rough and tumble play Bulbasaur evolves into Ivysaur.)

J: Bulby! Bulby!

A: Pika! Pika!

Thirty seconds later the camera is switched off as the rough and tumble turns into an actual fight.

Play with Pokémon games continued intensely for some years, with the GameBoy and Nintendo DS games returned to occasionally even when the boys were teenagers. A set of drawings produced by Alex at around the age of eight demonstrate an imaginative and processual engagement with the gameness of the Pokémon world, as well as its personalities and plots. The creatures are depicted, and new ones invented, as, at the same time, dramatic characters and as game elements. They are given the numerically valued attributes and abilities of the card and computer games. At times he created full sets of tiny cards, complete with a uniform abstract design for the obverse sides (Figure 4.2). There are echoes here of other spontaneous moments of meta-play, a playing with and framing of games and performances that appears to be fundamental to play (see Chapter 7, 'Real Worlds'), but which is particularly evident in play with the existing frames, screens and microworlds of contemporary media.

\section{Activity and passivity}

Our contemporary understandings of media and the childhood imagination are framed by a set of cultural distinctions between an active/creative or a passive/ derivative mode of engaging with imagination and fantasy. (Ito 2011, 493)

These distinctions between active and passive media are mapped across particular media forms and cultural practices, Mimi Ito notes. Drawing, performance, writing and so on are 'productive' and hence are considered 'creative, agentive, and imaginative', particularly when set against those that involve the passivity of 


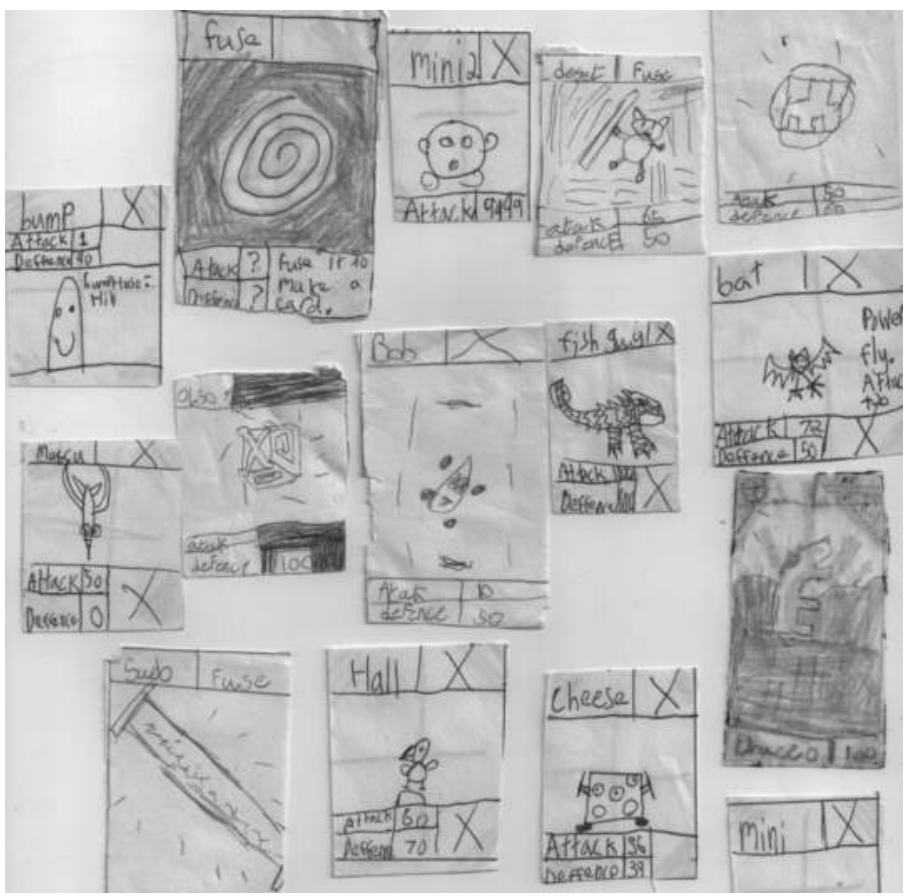

Figure 4.2 Meta-cards

the consumption of commercial media, specifically visual media, such as video game playing or TV watching (Ito 2011, 493).

The notion that children are passive consumers of media is familiar and has been thoroughly countered within media studies (e.g. Bazalgette and Buckingham 1995). However, there are three points worth making here that will be returned to throughout this book. Firstly, children's consumption of media across the digital (websites, video games), electronic media (TV, radio) and print media (books, comics) all requires the same imaginative and cognitive engagement to engage with stories, action, puzzles, jokes and so on. Secondly, though, there are material and mechanical differences too between media, notably between screen media, such as television and film, and interactive digital media, such as video games. As Espen Aarseth puts it, the 'cybertext' requires 'non-trivial effort' to open up its narratives and worlds (Aarseth 1997). The player of an adventure game must solve puzzles, make choices, fight enemies within the media text, not just watch or read these things happening. That is, there is another level or order of audience activity required with interactive media. Thirdly, and at the risk of sounding 
contradictory, interactive media also effect a distinct mode of passivity in their audience. Though the player engages with game as a semiotic machine, interacting with its world and characters, he or she is also in significant ways acted upon by the machine or system. In the circuits of input/output and stimulus/response, it is far from clear that the player is the initiator and controller of the gameworld, he or she is positioned and configured by the game's cybernetic and passional circuits (Lister et al. 2009, 398).

These circuitous questions of control will be answered in more detail in Chapter 5 ('Soft Worlds'). For now, I want to raise a question that is rarely asked in relation to media: what is wrong with passivity anyway? Many of the pleasures of media aesthetics and aesthesis (their effects on our bodies) are predicated on them acting on us. The rhythms, harmonies, forms, the play of expectations and revelations of visual art or music all play us as much as we play them. To be 'lost' in a film or book requires imaginative engagement but it also involves a delicious surrender or subjection to its structure, imagery, plot and character. Games are a key form here. By no means is all play phantasmagorical, straightforwardly imaginative, creative or poeitic. 'Activity' in media consumption for media studies generally means a more or less reflexive challenging or subverting of dominant meanings, but to play a card, board or computer game is to subject oneself and one's fellow players to the rules and the game as a system. Play with toys, cards, media can be repetitive, unchallenging, distracting, lazy. And often this can be deeply satisfying. For playful media such as comedy, getting the joke and laughing along is key - not reading against the grain or subverting it. And whilst game structures from board game rules to missions in GTA can be undermined, stretched, subject to parody and ludicrous performance, playing a game by its rules - accepting more or less the intentions of the designers and producers - is often, perhaps more often, more pleasurable, satisfying and sociable. Some games offer latitude for intellectual effort (chess), or individual style and flair, but others - for example, games of chance such as Snakes and Ladders - simply offer a system, a mechanic, a configuration of the players' movements and actions for its duration. ${ }^{1}$

Whilst much of this book is a celebration of imaginative agency within the rules or worlds of play, it also recognizes and explores the aesthetics of control and the circuits of action and passion. In not questioning the binary of activity and passivity, we can overlook significant cultural, cognitive and embodied pleasures, and miss key characteristics of particular media forms and behaviours. 


\section{Media materials}

we see how children co-opt a variety of ideas and images to inform or guide their play, culled from various sources such as personal experiences, books, television, songs, and movies. Children adapt these images to the playspace and opportunities available to them - in this case, adapting the traditional story of Snow White to accommodate the roles the players wanted to adopt and making use of the props at hand. (Dixon and Weber 2011, 484)

Video games, virtual media and transmedia systems all offer resources for imaginative play or expect and demand a creative engagement. These ecologies and their ludic forms allow a revisiting of the histories of children's media culture that highlights games over stories, and metamorphosis and transduction in the spontaneous worlds generated by play. They also require a rethinking of the nature of play in the contemporary media environment. They raise questions of materiality in the transduction of semiotic stuff into drawings, gestures, dance, objects and toys, and questions about the materiality of technologies in particular. Whilst the interactive consumption of digital media highlights their technological and machinic nature - in that they must be operated as much as watched or read - this does not represent an absolute distinction from predigital media. Rather it suggests that we relook at TV, films, books as media technologies that are also used, for play, for pleasure, for emotional or attentional modulation. The bedtime story is one familiar example of the book that can be rethought as a technology (linear narrative, illustrations, page-turning), with its associated technics of reading aloud and parenting (emotional reassurance, ritual marking of the transition to sleep, etc.). The advanced technologies of recent media forms invite us to rethink the operations of pre-digital media technologies and the events that realize them.

Finally, I have questions about the immaterial, but real, aspects of play, media and technologies, from the operations of game software to the operations of imagination. These will be addressed in the chapters that follow.

\section{Note}

1 Roger Caillois' spectrum of play is pertinent here: rule-bound games sit at the ludus pole, the spontaneous and improvized paidia at the other. It is important to note that Caillois does not privilege one over the other (Caillois 1962). 


\section{Soft Worlds: Play with Computers}

I like my screwdrivers like I like my hedgehogs: sonic (computer-generated joke). ${ }^{1}$

Alex watching Jo play the last boss battle in Lego Star Wars (21 May 2005):

A: It's not fair ... the computer never falls off does it?

J: Of course not - it's the computer.

A: The computer knows where it's going!

Video games and virtual media are fully part of a popular children's media culture generated by commercial and public sector broadcasters and producers. They have found a place within domestic ecologies of media use, both displacing and merging with entertainment and communication media from television to telephony through mobile devices and Internet channels. They resonate with, and amplify, previously peripheral media modes and genres: from board games to game shows, from playground jokes and games to collecting and swapping cards and toys, from the topographies and quests of children's books to reality television. Imaginative and pleasurable engagement with characters, environments, stories, powers and abilities, conflicts and quests flows between the everyday consumption of books, television, films, children's play and toys and video games. These flows are now solicited in the transmedial circuits laid down by popular media and toy producers and commercial playful media universes are generated from them.

But they are also quite different. This chapter will concentrate on another tangled line of descent, that of computer technology and media. The analysis of the video game as a computer-based medium demands the description of a distinctly new set of domains and agents. The focus will be on the computer in computer games, on the nature and behaviours of software, on real-time 
procedural operation, on simulation, interactivity and virtual environments. Games scholars have argued that video games are characterized by an 'aesthetics of control' (Murray 1997, Klevjer 2001). Evidently video games are 'interactive' media objects and as such their players exercise some level of agency in the on-screen events. However, the software that constitutes video games needs its own animate operations to be acknowledged in the dynamics of gameplay. Children and adults play with non-player characters (NPCs), artificial intelligence (AI), virtual physics and architecture, rules - but these artificial entities and processes are at play too. They respond but they also initiate they have their own coded and autonomous behaviours. To understand the gameworlds of video game play, we need an ethology of artificial as well as human players.

\section{Play with technology}

The history of children's play with technology is inseparable from the emergence of a commercial children's culture, discussed in the last chapter. In the nineteenth century, both everyday and exciting new machines were scaled down to fit children's hands: toy cars and guns, mechanical dolls and the domestic fittings and architecture of dolls houses. Educational toys and devices were popular, particularly those of scientific wonder and experimentation (optical toys such as zoetropes and kaleidoscopes) and engineering and construction. Some of these were intended for educational use, some purely for pleasure and entertainment, while others, like the home computers that would eventually follow, were used for both. The early twentieth century saw the first fantastical and modern machines of spacecraft and future weaponry - particularly for boys (Chudacoff 2011).

This overlapping of the functional and the symbolic, the mundane and the fantastical, the entertaining and the educational continues with everyday computer technology today, not least, of course, in the sophisticated toys that are the video game console and software. Younger children in particular are targeted by the manufacturers of computerized toys and devices, some like simple laptops aim to educate, others like the basic responsive robots Furby and Tamagotchi of the mid to late 1990s take more traditional toys and add mechanical life to that imbued by the child. Smart phones and tablets may also be given to small children, and there is a growing market for visual and tactile apps for a preschool market. ${ }^{2}$ 
The line between (hard) toy and (soft) digital game/character is not a clear one. The nurturing of a Tamagotchi was an ambiguous activity - was the eggshaped object itself the toy creature, or did the creature live inside it, looking out from its screen? There have been many video games since that used a similar game mechanic of attentive and repetitive feeding, and exercising, for instance, Pokémon, Nintendogs and Moshi Monsters. Interestingly, the human characters in The Sims are as much creatures to be nurtured in this regard as the cats and dogs of The Sims Pets expansion packs.

To date, a few particularly intense crazes notwithstanding, the virtual toys of video games have proved more successful than their actual, physical cousins. A Furby was sold on its responsive AI, its ability to learn from and communicate with its owner, yet it and other 'smart' toys like it were often played with as more traditional inanimate cuddly toys - switched off and incorporated into free play (Goldstein 2011, 325). The semi-autonomous nature of NPCs in many games, and the bots (software robots) of massively multiplayer online games (MMOs) such as World of Warcraft, ${ }^{3}$ is often at least as sophisticated as that of smart toys. Unlike smart toys whose behaviours and intelligence must stand up in comparison to the physical and social worlds in which they are situated, software characters often suggest a dynamic complexity beyond their often simple AI and repetitive algorithmic actions. As artificial agents in the artificial and ludic environments of a video game world, they are fully at home and set the terms of interaction, the rules of a game.

So, the functioning of video games is at once symbolic and technical. On the one hand, video games are toys, popular media, performative events, animated with characters and scenarios derived from established popular screen media. On the other, the analysis of video games as a computer-based medium demands the description of a special category of non-humans, software entities and agents depicted as individual characters, as collectives, or as aspects of the virtual environment itself, but all acting with a certain degree of autonomy. For instance, to play a video game is to play with, or against, artificial intelligence in the form of non-player characters and responsive simulated environments. This is a huge topic, so this chapter will identify and explore a few aspects and bodies that are most constructive in the gameworlds model. These include those that contribute to a game's 'worldness', its sense of a dynamic and responsive environment however abstract in appearance. These are components that constitute the newness of these new media for children, entities and operations that bring to children's play quite distinct experiences and demand quite different embodied 
and imaginative behaviour within play. So, whilst a computer gameworld may overlap with or nest within a literary or cinematic world (e.g. Harry Potter, The Lord of the Rings), it and its players are quite different entities to the readerplayer of the books or films. They are constituted by cybernetic feedback loops, have automata and non-human intelligent agents as playmates, all within and through synthetic and responsive playgrounds. They are part of what I will call 'distributed imagination'. Though the notion of 'world' evokes spatial dimensions, the computer gameworld may have as its underlying motive the simulation and manipulation of time, and of physical forces (friction, collision, gravity) for playful ends.

Video games as symbolic software systems have tremendous variety, from the abstractions of scale, space and objects in early arcade games such as Space Invaders, through side-scrolling platform games, top-down 'gods'-eye' strategy and simulation, to first-person shooters and massively multiplayer online games and back to abstraction with mobile games and puzzles. This chapter will necessarily be highly selective, referring back to the Lego Racers events, but with other examples that will draw out through comparison something of the variety and continuity of contemporary video games as cybernetic and selfacting systems.

\section{Rules and laws}

A fundamental formal difference between video games and most other popular children's screen media is that between rules and narrative. Whilst many games are framed by a dramatic scenario and enacted by fictional characters, the salient activities and pleasures of gameplay are driven by the abstractions of ludic conventions and systems engineered to sustain the flow of play or to facilitate competition and chance, puzzles and technical skill. In this regard, video games are more closely related to Snakes and Ladders than Snow White. The computerization of games changes the players' relationships to rules, however. Rules in board and card games, however strictly applied in the event of a game, can be negotiated, adapted and agreed before the game starts. They must also be learnt and understood. The game apparatus allows a variety of rule sets: limited perhaps on the Snakes and Ladders board, but with a deck of playing cards, infinite. Video games often allow a certain amount 
of adjustment to settings, difficulty levels and so on but in general players cannot adjust the underlying rules significantly. In fact it is often difficult to identify the rules as such. This is in part because the software itself monitors and adjusts key variables (it keeps the score, calculates strength and health levels and so on), and in part because the 'rules' are often inseparable from the world itself: where the character can go, what powers and abilities it has, relative strengths against enemies and so on. Even physical laws, for example, gravity, collision, friction - which are non-negotiable in the actual world - are coded into virtual worlds. This difference between the player's understanding and experience of a gameworld and its structure and rules, equating the latter to the 'laws' of the world, is explored by Michael Nitsche. He explains that through the design of the code, the programmer authors the rule-based space. Players may 'generate unique permutations' but only within the possibilities allowed by the code.

Players of a video game do not look at the underlying code but at dynamically generated visual and tactile results based upon it. They look at the mediated plane and see the performance of the code. The code itself stays hidden behind elaborate virtual worlds and interfaces, and the only time one might encounter it is when an error crashes the program and a debug message points to certain lines of broken code. Players do not have to understand the logic of the code but of the mediated game world. (Nitsche 2008, 25)

Well, this depends on what we mean by 'understanding. The player does not need to self-consciously read the code as code, to dig down into the layers of abstract algorithms below the elaborate audio-visual interface. But the mediated gameworld, the interface itself, is driven by the logic of the code and the player must also be driven by it. Helen W. Kennedy and I have explained something of the differences between popular narrative screen media and the processual worlds of games:

watch someone who is unfamiliar with simulation games playing The Sims for the first time. Building houses and naming characters is straightforward enough, but as the characters go about their daily artificial lives the tendency is to treat them as if they were real people, or at least as fictional characters who behave according to established conventions of narrative, and try to intervene accordingly. However the game's dynamics are driven by algorithms which rarely map directly onto any sense of actual (or fictional) human behaviour. (Giddings and Kennedy 2003, 160) 
The gameworld's laws and behaviours, the actions it allow, refuse, and insist on are all coded. They require that the player grasp and enact their demands and possibilities via the mediated world. As we saw with the Lego Racers, the game encodes the players.

\section{Interactivity and avatars}

Importantly, the [...] relationship between player and system/gameworld is not one of clear subject and object. Rather, the interface is a continuous interactive feedback loop, where the player must be seen as both implied and implicated in the construction and composition of the experience. (Newman 2002, 410)

The language of cybernetics in the study of children's popular media raises questions again of agency, activity and passivity. In contrast to the positive, active connotations of the term 'interactivity', it suggests instead a mechanization of play. If interactivity is regarded as an ideal mode of media culture for children and adults - the triumph of human agency in technological and virtual environments - its dark flip side easily conjures up fears of everyday and psychological colonization, or even science fiction nightmares of humans controlled by machines (Stallabrass 1993). In gameplay, as James Newman notes, the player is integrated into the circuit such that media studies' reassuring separation of the entities 'viewer' and 'text,' subject and object, is fundamentally compromised. In a similar vein, Lister et al. suggest that human movement in interactive media is prompted not by 'free will' but by the game-as-system; the player is not responsible for his or her own body, a body which is 'determined to move by being part of the circuit of the game. The player is acted on by the game - these are passional circuits (Lister et al. 2009, 398). Communication and control are inseparable aspects of cybernetic systems (Wiener 1965).

So video games are machines that articulate the visual and auditory aesthetics of popular screen media with a new 'cyborg aesthetics' in which the salient term is 'control' (Aarseth 1997, 55). This cyborg aesthetics is often most evident in the relationships between players and avatars. The avatar, as Diane Carr explains through her experience of the Tomb Raider games, is a machine through which the player enters into the game, and is thereby configured by the game:

as a player, I can do, undo and redo until Lara has effectively performed the challenge presented to her by the game, but I can only proceed through the game, through the space itself, if I perform the task as the game demands $[\ldots]$ 
A player will systematically experiment, and after trial, error and accidental death, correctly perform the intended and necessary response. The conditional terms of our motion through space are thus rendered explicit. We do not write our own narrative, we uncover a pre-existing text and conform to its injunctions'.

(Carr 2002, 174)

Game studies researchers with a background in media and film studies have applied and adapted theories of the film viewer's identification with key protagonists within a film narrative to the consideration of the gameplayer's identification with the avatar. The starting point is often the argument that the player may identify more strongly with the avatar because the player's control of the character's movements and decisions determine (depending on skill and experience) that character's ultimate fate (always within the strict limitations and possibilities structured by the game as software). Whilst this has proved a productive theoretical approach, it is limited by some significant misassumptions. One in particular is the assumption that 'control' is a linear connection, that the player has stepped through the fourth wall of the fictional/ media world to become at last the hero, the protoganist, the chief pleasure now one of control over the avatar and hence the imaginary world. As Diane Carr makes clear, control is key to videogame aesthetics but it functions at least as much through acting on the player as through players acting on the world, a reciprocal configuration.

For example, it is often taken as given that psychic 'identification' in both film-viewing and gameplay is the most significant mechanism in the engagement between technological/media artefact and human viewer or player. Whilst playing with or through a favourite character offers new media pleasures, any sustained engagement with gameplay soon makes cognitive and technical demands on the player that go beyond any straightforward notion of deepened imaginative identification with the character (again this was evident with the ambiguous drivers of Sandy Bay). On one level there is the simple fact that playing a video game is a complex technical, cognitive and embodied feat. Initial delight at 'controlling' the avatar soon comes up against the game's demands for adequate action in steering, jumping, fighting, solving puzzles and so on. It has often been observed that players will slip between the first and third person as they account for their virtual activities. They demonstrate eloquently the shifting or intermittent nature of attention to the avatar. At times Mario is a character in the sense of a cartoon character. The player may prefer to play as Mario for reasons bound up primarily with questions of taste, preference or aesthetics. But Mario is only occasionally the game's protagonist as a film scholar might 
understand the term (a character), more often he is, as Fuller and Jenkins have suggested, effectively a cursor, a marker or tool with which a user intervenes in software:

In Nintendo"s narratives [...] The character is little more than a cursor that mediates the player's relationship to the story world. Activity drains away the characters' strength, as measured by an ever shifting graph at the top of the screen, but it cannot build character, since these figures lack even the most minimal interiority. (Fuller and Jenkins 1995, 61)

Since his early appearances in Donkey Kong and Super Mario World Mario has become a technically more complicated entity, but his nature retains something of the cursor in his mediation of player input and gameworld activity. In more recent games, like Super Mario Galaxy, he collects items that augment him with new, temporary capabilities and attributes. These power-ups might be figured as special costumes: a Bee Suit bestows the power to hover, a Spring Suit to jump particularly high and so on. In turn, these powers are carefully designed to allow access to new sections of the gameworld, as much a playful and temporally determined set of keys or codes as either a cursor or character. Characters rendered with the look and role of the cinematic protagonist (whether action hero or cartoon character) are, fundamentally, software entities. Solid Snake, from the game Metal Gear Solid, for instance, is

embodied by the player as a set of available techniques and capacities [...] In fact, it does not make sense to even talk about 'he' on-line, because there is no 'he' as distinct from the engaged player, like Lara Croft or Sonic the Hedgehog [...] on-line, 'Lara' is the player's ability to run, jump, shoot ... while 'Sonic' is speed, looping-the-loop, collecting rings.... (Newman 2002, 413)

Here, then, avatars are not virtual puppets suspended before the player in the exploration of their worlds; they are semi-automata acting and performing beyond the direct control of the player (Sonic's blistering turns of speed propel him beyond the control of the player, Lara's elegant jumps and somersaults are merely suggested by the player with the press of a button). They are parts or components of the game, hybrid media-automata, entities that at once offer visual/kinaesthetic pleasure and instrumental progress through the game system by means of their programmed capabilities and limitations:

the ludic and vehicular nature of avatars is articulated with their status as symbolic objects. Again the dynamics of effect and affect are inseparable [...] much of our pleasure in the affordances of Yoda and Chewbacca in this game 
[Lego Star Wars] lies in the articulation of the possibilities for movement and action they afford on the one hand and in the visual appeal and expectations that come with their status as both familiar media characters and Lego toys on the other. This articulation of course shifts through the rhythms of the gameplay. In the heat of a battle or the tangles of a puzzle the player may be less concerned with the appearance or intertextual connections of his or her avatar, but at other moments these factors may be primary, and at time the two will be inseparable. (Giddings and Kennedy 2008, 25)

Different games and game genres establish different connections between the game's processes and the possible actions of the player, eschewing the anthropoid avatar as mediating device. The player of The Sims does not control their virtual family directly, but merely attempts to direct them. Some games, such as SimCity or Age of Mythology, present multiple characters and roles to the player. The virtual people of SimCity are a central game element (they will riot if taxed too heavily, or if inadequately policed) yet are 'invisible'. They are an example of the profoundly algorithmic characteristics of all game entities in that they are only the interface's presentation of the effects of the interactions between a set of variables over time, variables controlled by the computer, though allowing some intervention by the player (Manovich 2001).

Gameworlds are computer systems some of which allow emergent behaviour, but only that facilitated by their always systemic, mathematical logic. The Championship Manager series, for example, with its emphasis on the revenue, tactics and transfers of football as a business - over the playing of matches themselves - is often jokingly referred to as 'playing a spreadsheet.' If we think of game software, then, as software, a set of tools or an environment of virtual tools and processes, rather than a fictional world, then player behaviour starts to look quite different.

...while creating a zoo for different type of cats (e.g., tigers, lions, and leopards), Rachel learned of a new birth in her zoo and responded by selling the newborn animals immediately [...]

1. Rachel: Yeah, because the baby - um because there was a birth of an endangered animal? $\langle\ldots\rangle$ that's really great. And it's especially great for me because I can sell the little baby for $\$ 1000$. [Clicks button to sell baby.] That's a good deal.

2. [Rachel pauses, then explains herself.]

3. In real life? I would not own a zoo like - I would not, um, what would what would you say, um, manage a zoo like this. I would be much more caring. But in this game, [smiles and nods her head] I'm more (.) greedy. But that's okay. 
Okay, so now that I have more money, I actually can (.) that's really good. Except it will go very quickly. (Stevens et al. 2008, 59-60)

In a study of the entertainment and educational ecologies of children's video game play, Reed Stevens, Tom Satwicz and Laura McCarthy followed two girls' differing approaches to the simulation game Zoo Tycoon. They note a complex set of relationships between the girls' cultural experiences and tastes, and the laws and dynamic structures of the game, resulting in distinct play events that cannot be reduced to either. One of the girls, Katarina, enjoyed decorating her bedroom, making lamps and designing websites for school projects. Rachel, on the other hand, led a very busy life that required an efficient organization of school, homework, church, sport, babysitting and social activities, all carefully set out on a calendar. Katarina's enthusiasm for design, the researchers note, was clearly connected to her behaviour in the gameworld, as was Rachel's 'preference for efficiency'. Simulation games such as Zoo Tycoon are often open enough to allow the development of a visually attractive virtual environment as a player's main aim (Katarina), or conversely, the coordination of a well-organized world maximizing growth and other economic gains (Rachel).

Katarina and Rachel arrive at zoos that look and function differently [...]

We found that Katarina played the game to 'design' and reported that she learned what she called 'design methods'. Rachel played to efficiently beat scripted scenarios given by the game. Whereas Katarina sought to make her exhibits 'pretty', Rachel arranged her exhibits for best functionality rather than aesthetic appeal. (Stevens et al. 2008, 59)

Though often referred to as 'sandbox' games, these worlds do not have the freedom and plasticity this implies. Katarina's aesthetic approach, if pursued without attention to her zoo's business operations, risks economic failure. For instance, she spent her in-game funds on trees and rocks and only the minimum on feeding and caring for the animals. These game systems are more virtual economies than a translation of an actual toy zoo. Unlike the more open construction possibilities of, say, Minecraft, most simulation games have a carefully balanced set of environmental and economic laws to constrain possible actions for ludic ends. Play in both SimCity and Zoo Tycoon is sustained by the need to gradually accumulate resources to expand, develop or ornament the gameworld. So, whatever aspects of the players' taste, imagination, or sense of self and identity are mobilized in video game play, their behaviour in the gameworld is always transducted by the code. 
This inevitable process of transduction across actual and virtual play is neatly illustrated by the technologically mediated morality of the 'cheat code'. Simulation games always contain these more or less hidden 'cheats', embedded codes that allow the player to generate infinite funds bypassing the 'preferred' gameplay route of gradual and skillfull accumulation. ${ }^{5}$ Rachel chose not to use cheats, a decision apparently driven by both virtual play preference and her actual world moral order. The rejection of cheats reflected 'her overall orientation to life and game play', and once she had tested out the game as a system, she chose instead to abide by the rules and their implied morality and 'clearly sanctioned modes of improvement'. As Rachel herself put it,

Before I did the scenarios I definitely used the cheats a lot...the money cheats ... for the scenarios I feel like - I don't know. When I was doing more free games ... the thing that would get in your way of having a really nice zoo was not having enough money. So in the scenarios I try to - I don't know, work harder at being better. (Stevens et al. 2008, 56)

Katarina on the other hand 'was so unwavering in her focus on using the game as an aesthetic design medium that she actively occluded in-game instructions that recommended efficiency and point-maximizing behaviors', and had no qualms about using cheats to ensure the survival of her zoo (Stevens et al. 2008, 55).

To reiterate, I would argue that what we are seeing here is not a straightforward playing out of children's own personalities and fascinations as assumed in the therapeutic world-building of actual sand play (Lowenfeld 2008 [1935]). There are complex relationships between these girls' senses of themselves and their behaviours in the game. The game is not a virtual tabula rasa on which to project an identity, rather a playing out of certain expectations and preferences through choices in the game, and the directing of those preferences into its algorithms and economies. Rather, these are loops through which the gameworld as a computer system modifies and regulates cultural and subjective preferences, imagination and creativity according to the logic of an economic and behavioural simulation. The game event is a cybernetic system in which the actual, biological, livedcultural and the mathematical, artificial and ludic circulate and shape, govern and exercise varying degrees of control over each other. It works on, shapes and extends imagination and identity, distributes them around a network of human and non-human bodies and processes.

I'll return to this notion of the cybernetic in particular as constitutive of everyday gameworlds later in this chapter. In the meantime, I will continue with related questions of agency and action within and around virtual worlds. 


\section{Lively worlds}

The computer-generated environment of a video game in its entirety is an animated virtual playworld. By concentrating on the avatar we might miss the active, agential nature of the gameworld too, all generated by the same software engines, rendered with the same polygons and texture maps, ascribed behaviours and affordances. Players can manipulate a game's topography (Super Monkey Ball), or construct it (The Sims, Little Big Planet, Minecraft). In more abstract games, there is no clear symbolic distinction between environment and characters (Tetris, Minesweeper). We have already explored something of the world of Sandy Bay, its ludically motivated landscape and physics. As I have hinted earlier, to play a video game is often to play with the whole gameworld as a system.

Gameworlds are virtual polities as well as virtual geographies: as Rachel and Katarina implicitly recognized, they simulate social and economic systems. The society and economy might be explicitly figured as a gameplay motive - in The Sims, Zoo Tycoon, Animal Crossing - or they may be similarly integral to the game mechanic but adopt a different frame of reference. To play any game that involves the accumulation of points, coins, rings and their expenditure for survival, items, power-ups and so on is to be subject to a virtual economy in all but name.

Perhaps the most significant - but rarely studied - feature of video game worlds as virtual media is the strange socialities they generate between players and artificial intelligence and non-player characters. Artificial intelligence is perhaps the most commonly understood instance of simulation as autonomous agent in digital games. In a game AI generally refers to the components of the program that respond most sensitively to the actions of the player. The term covers both the coding of the behaviour and responses of NPCs and the overall sense of the gameworld as a system that is responding convincingly to the player's engagement with it. Generations of children have grown up playing with automata, software robots, whose behaviours are driven by the application of AI to entertainment. This 'expressive AI' is about

creating a sense of aliveness, the sense that there is an entity living within the computer that has its own life independently of the player and cares about how the player's actions impact this life. (Mateas 2003)

In some applications of AI to playful media, such as smart toys or chatbots, the artifice is unavoidable and prompts ontological reflection on the nature of life 
and reality. Sherry Turkle's research with computer toys from the late 1970s illustrates this: she found that children playing with computers and interactive toys were also playing with their understanding of machines' agency and intentionality. An interactive toy that seemed to anticipate the child's moves or strategy would be angrily accused of cheating, for instance. A nine-year-old girl with some experience of programming compared the vitalism of television and computers:

'The television set isn't alive. It doesn't make up its picture. It only passes it on.' A person, she explains, might have to tell a computer how to make a picture, but the picture doesn't exist in the world before the machine gets involved: 'The computer has to know how to do it. To make it up.' This reasoning leads her to a special kind of verdict for the computer: 'It's sort of alive'. (Turkle 1984, 41)

This sort-of life resonates with all the sort-of realities of play, games and the virtual: in her instrumental engagement with heterogeneous entities in her world she has established a practical variegation of agency and autonomy, rather than the binary opposition of human subject and non-human objects (nature, technologies) that has structured Western thought since the Enlightenment (see Lister et al. 2009, 277-280).

The sense of aliveness or intelligence (or at least the sense of plausible behaviour and responses) of NPCs seems to require a much lower degree of sophistication or realism than that expected of chatbots, smart toys and other more serious AI applications. Within the stylized gameworld, and when put to playful ends, automata can seem perfectly acceptable in their animated artifice. An influential essay by the philosopher Daniel Dennett offers productive ways of thinking about the imaginative/pragmatic relationships between human and non-human players. Taking a chess computer as his example, his argument runs as follows: the strategies of a sophisticated chess machine are so complex that they cannot be predicted by a human player. Even the programmer couldn't say what sequences of moves it would make in a particular game. Hence, it is only possible to play chess with a chess computer by ascribing intentionality to it, by reacting to it as if it were an intelligent player:

when one can no longer hope to beat the machine by utilizing one's knowledge of physics or programming to anticipate its responses, one may still be able to avoid defeat by treating the machine rather like an intelligent human opponent. (Dennett 1971, 89)

This is the 'intentional stance', and Dennett distinguishes it from the 'design stance' in which a detailed knowledge of how the computer or program is 
designed would allow the designer (the user or player) to predict the system's response to any input or operation. In the case of chess, the design stance would entail the player knowing enough about the instructions coded into the game-as-program to definitively predict every move the computer would make (Dennett 1971, 87-88). Yet,

on occasion a purely physical system can be so complex, and yet so organized, that we find it convenient, explanatory, pragmatically necessary for prediction, to treat it as if it had beliefs and desires and was rational. (Dennett 1971, 91-92)

Dennett offers this concept as a practical, pragmatic way of understanding the operations and agency of complex systems that at once acknowledges the very palpable (and perhaps unavoidable) sense of engaging with a system as if it had desires and intentions, whilst rejecting naïve or idealist versions of anthropomorphism:

The concept of an Intentional system is a relatively uncluttered and unmetaphysical notion, abstracted as it is from questions of the composition, constitution, consciousness, morality, or divinity of the entities falling under it. Thus, for example, it is much easier to decide whether a machine can be an Intentional system than it is to decide whether a machine can really think, or be conscious, or morally responsible. (Dennett 1971, 100)

So this intentionality does not assume that complex systems have beliefs and desires in the way humans do, but that their behaviour can, indeed often must, be responded to as if they did. Or perhaps, and Dennett hints at this, their 'beliefs' and 'desires' are not so much metaphorical as analogical. The intentional stance usefully sidesteps the speculative cul-de-sacs of arguments over machine consciousness and allows an ethological attention to what complex systems, in this case software, actually do: what behaviours they exhibit, what effects they have.

This 'unmetaphysical' notion of the intentional system both resonates with Latour's non-human delegations and suggests ways in which we might theorize our material and conceptual engagement with complex computer-based media. It might suggest why children interacting daily with artificial intelligences and autonomous agents in video games do not seem to reflect so deeply on the ontology of their playmates as did the players with early computational toys observed by Sherry Turkle.

To give a simple example from a more recent computer game form, the player of a first-person shooter (FPS), such as the Call of Duty series, must respond to the behaviour of the enemy NPCs as intentional. These automata 'want' to kill 
the player's avatar and avoid being killed themselves. Metaphysical reflections on the nature of machine intelligence are neither here nor there: in the gameplay moment the enemies move, target or evade as intelligently as their limited but effective range of behaviours requires.

Mateas cites the behaviour of the ghosts in Pac-Man as a foundational moment in the development of expressive AI. The ghosts do not simply hunt down the avatar as quickly as possible, which would be easy to program but would result in a very limited game. Rather, each ghost has its own simple coded behaviour. One chases Pac-Man directly, one is directed to a point immediately in front of Pac-Man and so on. In addition they attack in waves, gradually attacking more often. Individually none of them is a particular threat, but the combination of simple behaviours generates a non-linear and dynamic ludic environment. The ghosts' AI must be both complex enough to deny the player a 'design stance' the ability to predict their movement and so easily avoid them - and controlled enough to facilitate an exciting game:

This behavior must challenge the player without being impossibly difficult, and be unpredictable enough to make the ghosts feel alive and responsive to the player's activity. (Mateas 2003)

It is not only individually figured characters that must be ascribed intentionality but also 'intelligences operating behind the scenes'. For example, the player of a real-time strategy game (RTS) might 'sense' the presence of the enemy commander - or even the game as system itself - in the actions of computer-controlled troops. In this case we are closer again to Dennett's chess machine; it is not the knights and pawns that are played against as an animate entity, but again the computer system itself as player, the one that makes the Lego Racers go.

From the uncomplicated but highly effective behaviours of Pac-Man's ghosts, the tactical behaviours of military units in Advance Wars, to the convoluted conversational exchanges of the citizens of Animal Crossing, everyday play is now populated with simulacra. The initiation, prediction and anticipation of and reaction to - the behaviour of software entities (called 'intelligent agents' in less playful computer systems (Wise 2011)) are quasi-social and technical practices now are fully embodied by young children.

If contemporary video games appear to leave little space for immediate reflection on the life or intelligence of the game characters, this is due in part, no doubt, to the intensity of attention and reaction demanded by actiondriven games - there simply isn't the time. It may also be due in part, as 
suggested earlier, to the mediation of the NPCs through their unnatural ecology - that is they are more at home in the sort-of world of the game than entities such as Furby are in the actual world.

Regardless of whether children reflect on the virtual life in their play or not, as players they must - to play the game at all - adopt the intentional stance and 'engage the machine intelligence as machine intelligence (rather than as a pretense to human intelligence signified by the avatar simulacrum of a human...) (Simon 2007, 168). The player must accept the automata as sort-of alive, credit their non-human behaviour with a level of logic or intentionality entirely appropriate for the task in hand. Often this is simple enough in principle, if trickier in action - avoiding, again, the FPS enemies for instance. Sometimes the player must try to work out in more detail what the enemy might do, what its encoded behaviours are. In the more strategic FPS Call of Duty 2, Bart Simon points out, survival on the cinematically rendered D-Day landings relies on understanding and working with the behaviour of the computer-controlled comrades:

It becomes clear after dying the umpteenth time that sussing out the mechanics of the coop AI is crucial; you must move as a group, you must wait for cover fire, you must protect your mates, etc.... There is almost no dialog here, your comrades do not pretend to be able to hold a conversation in the trenches, instead there is what I call a 'conversation of actions' and the increasing recognition that you must keep 'face' with the $\mathrm{AI}$ in order to effectively play and make meaning of the game. (Simon 2007, 168)

Simon's account could be considered a playful, experimental ethnography of a synthetic society, a society that is uncomplex and singular in its relationships, behaviours and goals, but still dynamic and reactive. Detailed description of it as a social group or event highlights the profoundly machinic strangeness that underlies the photorealistic imagery:

In one epic scene you are a Russian private storming a German held railway station in Stalingrad. You begin the scene crawling through pipes on your own and drop down into a room full of comrades in a fire fight. There is a sense that they know what's up and you take a moment to get your bearings then you move and a group breaks off to follow you. Depending where and how fast you move your comrades will take up positions nearby. If you move too far too fast you are on your own. You cannot direct the troop with menu commands (you are not in control in that sense) rather you must in a sense spend some time learning the algorithms that govern the movements of your comrades. The action is meant to be cinematic and thus a re-mediated Spielberg experience 
[clearly capitalising on recent popularity of Saving Private Ryan and its visceral beach-landing scenes] passing itself off as realistic but that feeling is mitigated by the strange inhumanity of your comrades ... they don't speak, they do not appear as individuals, there is an endless supply of them. And yet, they act. They act on behalf of an AI module just as your avatar acts on behalf of you. The AI is tracking you and modifying its avatars' actions and you must learn to track it. This mutual tuning is done through action in the game, a conversation of actions; and once you find the rhythm combat is a cinch and the feeling when combined with uplifting music is euphoric. (Simon 2007, 168)

Simon compares software chatbots and their attempted simulation of human conversation - and the derision they receive - to the often very technically limited 'AI' of games (often simple dialogue trees). The game structure, trajectory and abstractions facilitate an ease and naturalization of conversation (and conversation of actions) - the non-human nature of these soft playmates is accepted as part of the flow through the synthetic, conventional gameworld:

Coop AI opens up the field of exploration in a way that Turing test driven competitive AI has all but shut down. (Simon 2007, 169)

The Animal Crossing series of video games for various Nintendo consoles illustrates beautifully this opening up of cooperative AI for play. It also invites reflections on the temporal or durational dimensions of virtual gameworlds. Though very different in appearance, pace and gameplay from an FPS, like the FPS the 'social' world as a ludic event is entirely bound up with speed and rhythm. As a resident of a small village populated by animals, the player must, over days, months, even years, build and maintain relationships through conversation, buying and selling items and exchanging letters and gifts. Everyday interactions are rarely dramatic, often just a simple exchange of pleasantries. The player communicates through the selection of a question or response provided by the game as short lines of text. This minimal conversation may at times intervene in a significant event in the game, or it may simply be - like so much actual everyday conversation - an affectual exchange, phatic communication to sustain relationships and 'community'. An example of the former is when an animal tells the player's character that they are thinking of leaving the town. The player is given two or three options in a simple dialogue tree, generally a plea to stay and one or two degrees of indifference. The appeal to stay will generally result in the animal's pleased surprise that the player's character cares so much and an emphatic decision to remain in town. It is not clear whether the general 'shooting 
the breeze' chat has any instrumental role in maintaining the general happiness of the animals, but the game strongly suggests that it does, and it seems to feel like it to the player. $^{6}$

\section{Virtual time}

Late December 2008, between Christmas and New Year, a liminal hiatus in the hard work of festivities. I'm waiting for a knee operation and can't walk far or drive so we are mainly stuck indoors. We spend much of our time in a series of virtual worlds.

Alex, Jo and I are in the lounge. Alex is playing his 'file' on Jo's Animal Crossing: Let's Go to the City on the Wii, Jo is playing the latest Pokémon game on his DS, but helping Alex when needed. I'm intermittently playing The Legend of Zelda: Phantom Hourglass on my own DS. The boys go off into the other room for their tea (I can't help their mother much, so she is the only one not playing). The Animal Crossing game is set to 'pause'. Its background music tinkles away quietly, an animated sheet of house symbols scrolls endlessly and impossibly smoothly in the flat background, whilst a ticker-tape message slides past horizontally, pointing out - to no-one - that connection with the wiimote has been lost. I'm stuck in a labyrinthine level of Phantom Hourglass, and because of my knee, I cannot sit at a desk and check an online walkthrough on my laptop, so my gameworld too is paused, on 'powersave', animation suspended. Alex sticks his head round the door to check the clock on the mantelpiece. He has been invited to visit by Rod, one of the animals in his village, at 5.45. As the gameworld is synchronized with the actual time - virtual time ticks away even on pause - Alex doesn't need to check the game itself. He has time to finish his tea before restarting.

Gameworlds unfold in virtual time as well as space, and their temporalities find rhythms with those of the mundane world just as virtual space interpenetrates everyday space. Game systems manipulate time in diverse and exotic ways to fit or drive gameplay. A game of football in FIFA 13 looks very much like the (televized) real thing but the 90 minutes of the actual game - displayed on the screen - flies by as players choose its duration (between two and ten minutes for each half). A turn-based strategy game, such as Advance Wars, has infinite patience when it is the human player's turn to deploy military units, whereas a real-time strategy game has its impulsive temporality writ large in its name (see Juul 2004). Sonic the Hedgehog famously faced the player and scowled, 
tapping his feet, if not immediately driven through his loops and platforms; other games have time limits to levels, or NPCs that will act on the player's avatar if it does not act itself. Whilst early adventure games would remain completely inactive between moments of human input, later games - particularly simulation games - once started will blithely play themselves until further human input is received. ${ }^{7}$ Time in SimCity or The Sims can be set to fast-forward as decisions in the modelling of economies and behaviours are tested in imaginary time. Most games can be paused, or frozen at a save point, to be reanimated later.

Animal Crossing's charm and gameplay are predicated on its simulation of real time.

Amelia (an eagle), asks Joey (Jo's avatar in Animal Crossing: Let's Go to the City) to take a present to Aurora (a penguin) by $3 \mathrm{pm}$.

$\mathrm{S}$ (checking my watch): You've got 20 minutes then.

J: No, here it's 25 to...

The game is set to the internal clock of the video game console, so - if the console clock is set correctly - night, day, seasons, festivals and birthdays are marked throughout. Alex was allowed one year to stay up until midnight on New Year's Eve to see the celebrations in his Animal Crossing town. He was barely awake when the virtual townhall clock finally struck twelve (about five minutes before the explosion of noise in the actual street outside, suggesting virtual and actual time were slightly out of sync). A small group of animals gathered before a 'Happy New Year' banner, were addressed by the tortoise Mayor and watched a firework display. It looked anti-climactic to me, but Alex went to bed satisfied he had attended a real event.

The clock and the world's temporality in Animal Crossing are completely integral. The clock isn't a measure of time, but virtual time's arrow itself, driving forward the events, economies and relationships, not just ticking along beside them. The game has some elaborate measures built into its fiction to avoid temporal paradox, manipulation or collapse. For example, in the 3DS version, Animal Crossing: New Leaf, the player can buy turnips once a week from a boar called Joan. These can then be sold at a profit later in the week (the player must check the turnip prices each day - that is every actual day of the week to determine the best time to sell). A player could quickly build up funds by repeatedly resetting the world's clock to effectively 'fast forward' from Joan's arrival to the optimum market conditions. However, the gameworld's artificial- 
natural laws foreclose this market manipulation and, as Joan explains, if the clock is changed, the turnips immediately rot and cannot be sold.

Animal Crossing's temporality is integral to its social and economic systems too, which are in turn the basis of its gameplay:

The game design intentionally draws on the passage of time to create both emotional resonance and economic value in the gameworld. (Kelley 2007, 181-182)

The game is a virtual economy through which natural resources, commodities and affects flow. The player gathers natural resources for exchange for currency ('bells'): fruit, shells, insects and fossils are shaken from trees, beach-combed, caught in a net or dug out of the ground. Though the buying and selling of furniture, ornaments and natural resources in a series of little shops gives the impression of an economy of mercantile capitalism, it is in effect a simulation of pre-capitalist symbolic exchange in the anthropological sense. Rather than accumulation for its own sake, exchange here follows the logic of a gift economy (Baudrillard 1993, Mauss 2002 [1950]). Animals ask a favour (to supply an apple or a particular type of fish) and then reward the player with an object (an item of clothing, furniture or ornament), or they often respond to a visit or kind word from the player with a gift. Both pleasantries and objects flow between characters, cementing relationships and the virtual community. The bells accumulated by the player from these transactions are either fed back into the community through public works (park benches, bridges) or are spent on personal adornment or on enlarging and decorating the player's house, in an odd mix of municipal socialism and potlatch performativity.

The circulation of affect is not constrained to the virtual world. Happy animals - who often sing and dance when especially pleased - delighted Jo and Alex, for example, when the boys had remembered a character's birthday (again in actual time, once a year) and visited its house with a present. Conversely, Jo once inadvertently opened a present he had been asked by one character to deliver to another and was upset almost to tears by the donor's angry disapproval.

This is an economy driven primarily by affectual circulation- gift-giving, deliveries, writing and posting letters, short pre-rendered and often surreal conversations with non-player characters - the collusional flow and circulation of virtual objects initiating and sustaining relationships (and actual-world emotions) through exchange and gifts, flattery, the coining of new nicknames 
or characteristic greetings, delivery of presents for others, the finding of lost items. And gifts are -as anthropologist Marcel Mauss would approve - always reciprocated.

\section{Everyday cyberculture}

Theoretical work on digital culture in the late 1980s and 1990s dramatized the future potential and import of emerging new technologies and media of VR and computer-mediated communication (email, bulletin boards, playful spaces such as Multi-User Domains) through a technological imaginary inspired by science fiction, and in particular the subgenre of cyberpunk. My descriptions of contemporary digital culture as cybernetic, virtual, simulacral, as intense intimacies between the human and the machine, echo this rich body of ideas. However, my descriptions in this book are neither futurological nor of an imaginary order. Contemporary virtual and networked media events are cybernetic, simulational even if utterly mundane: to play in the whimsical cyberspace of Animal Crossing or the persistent virtual server-worlds of World of Warcraft is to actualize William Gibson's famous fictional dataspace. As the futurological frenzy that attended cybercultural studies evaporates, it is important to note that it is now quotidian reality.

Like the virtual, the 'cybernetics' of cyber-culture is often used in a vague way to denote media and behaviours in and through digital networks. Here I want to insist on the importance of its precise uses and history, particularly as conceptualized by Norbert Wiener as the circuits of feedback of information and control within systems, where systems might be within a machine (e.g. a steam engine or computer) or might consist of machines and humans or other animals (Wiener 1965 [1950]). I will also touch on Gregory Bateson's subsequent synthesis of cybernetics, pyschology and anthropology for thinking about systems beyond those contained within the black or beige box of the game console (Bateson 1972).

In their influential and encyclopaedic book on game design, Katie Salen and Eric Zimmerman devote a chapter to games as cybernetic systems. A cybernetic system, they explain, contains three elements:

a sensor, a comparator, and an activator. The sensor senses something about the environment or internal state of the system. The comparator decides whether or 
not a change to system needs to be made as result of sensor's reading, and the activator activates that change. (Salen and Zimmerman 2003, 218)

This simple model might be of a closed system, monitoring and modifying its own internal state, or it might be part of, and interacting with, a larger system. Thermostats in a central heating system are a commonly used example. The thermostat senses a room's temperature, compares it to its settings and activates a change when the settings are reached (i.e. it turns off the heating when the preset temperature is reached). A computer gameworld is built from units of this simple triadic logic:

As a cybernetic system, the rules of a game define the sensors, comparators, and activators of the game's feedback loops. Within a game, there are many subsystems that regulate the flow of play, dynamically changing and transforming game elements. (Salen and Zimmerman 2004, 218)

A gameworld can be thought of a system, but it is one itself made up of interrelated subsystems. These subsystems might control a particular relationship or behaviour, but are always connected:

A cybernetic system within a game that directly affects just one component of a game will indirectly affect the game as a whole'. (Salen and Zimmerman 2004, 218)

They give the example of a cybernetic feedback subsystem engineering an optimum play-state in racing games. The futuristic racing game WipEout tracks the speed and ability of the player through the behaviour of the virtual vehicle and adjusts the speed of the computer-controlled hovercars accordingly. A racing game where opponent cars disappear into the distance or where they are left standing is no fun (unless of course, as we've seen, other games can be improvized), but a cybernetically modulated cluster of vehicles just fast enough to present a challenge is. In the Mario Kart series of games (particularly popular in our house), this cybernetic levelling out of the differentials of skill and luck is effected through the targeting of power-up items according to the players' (human and computer-controlled) position in the race. The leader will, as he or she bashes through a power-up box on the track, collect a green shell, a kind of unguided missile and the least powerful item in the game. There is a range of devices and powers worthy of Acme Corp. in a Looney Tunes cartoon to be collected - according to the kart's position in the race, down to the 'Bullet Bill' which the player in last place may, occasionally, receive. For ten seconds or so 
the kart is transformed into a large, fast invincible missile with painted teeth like a Spitfire. Acquiring one of these towards the end of a race can shift the driver from last to near first place. It is a colourful and witty animation of power and action, but serves an underlying cybernetic principle of system regulation put to ludic ends. As Grahame Weinbren puts it,

The point of the game [...] is to keep the player between two frontier zones on the verge of mastery, on the verge of losing control [...] The pleasure is in overcoming what was just a few minutes ago insurmountable. (Weinbren $2002,183)$

The gameplay experience is a carefully designed experience, one that is achieved only when the human player accepts, learns, trains for the machine's demands. It is a tightly engineered circuit.

Computer and video games, then, are cybernetic systems for popular entertainment, 'communication systems where the functional differences among the mechanical parts play a defining role in determining the aesthetic process' (Aarseth 1997, 22). For Aarseth, a cybertext

shifts the focus from the traditional [media/literary] threesome of author/ sender, text/message, and reader/receiver to the cybernetic intercourse between the various part(icipant)s in the textual machine. (Aarseth 1997, 22)

It also complicates any simple conception of interactivity as a two-way flow of action and reaction between a human and a computer application, a player and a game. It multiplies the agents at work in part by breaking these agents down into their components.

This control over gameworld information, reminiscent perhaps of the detective novel's careful timing of revelation, is integral to many games, and hints at the autonomous 'intelligence' of the system as experienced in play. The player only sees or knows aspects of the gameworld, its algorithmic operations and coded totality are fully known only to the system itself, and revealed only partially. Sometimes this is ignorance of the precise systems of causality (as in Animal Crossing's relationships), in other more expansive games it is spatial, that is the player can only see and interact with the area of the gameworld they are currently in. In many games, from the early arcade game Defender to real-time strategy games such as Age of Mythology, adventure/RPG games like Legend of Zelda and MMOs like Guild Wars 2, this lack of knowledge is integral to gameplay, requiring a restless exploration of 
labyrinthine dungeons, or perpetual vigilance and reconnaissance as enemies spawn and gather strength in remote regions.

The video game (and to varying degrees other software applications) not only configures its player through the technical possibilities and constraints inscribed during design and manufacture, but it is also designed precisely to configure its player continually during play itself. Gameworlds and temporalities, modes of presentation, puzzles and combat, engagement with computer-controlled characters are all constantly impinging on the player's experience and responding to the player's responses. Each different game, and each different playings of any particular game, rigs up different connections and circuits and demands different states and different pleasures - visual and audio cues, spatial conventions, embodied memory, the cognitive intensity of puzzle solving and the interludes of visual pleasure or calm. And far from offering the player a virtual world within which to exercise his or her interactive mastery, they invert this agential relationship: the game generates and navigates its world, the player is prompted to respond. The player's sense of intense agency in the gameworld is not illusory, but it is circuitous rather than initiatory, exercised through simple signals to be processed in synthesis with other subsystems: a twitch of a thumb on a tiny button triggering dramatic screen action is, in cybernetic terms, an amplification by the circuit.

\section{Control aesthetics: I'm the one who makes the Lego Racers go}

To tease this out a little further I will use it to revisit the Lego Racers events. Firstly, the interplay between the children's pleasure in the animated mini-figures and the virtual building of cars demonstrate this assertion that a computer gameworld must be understood as a cybernetic system put to the service of playful media, processing and articulating both interactive control and popular media aesthetics. Secondly, I will suggest that this machinic play produces a distribution of imagination particular to computer play.

Video games configure their players, in real time, a moment by moment, movement by movement, response by response anticipation of behaviour. Ludic futures are built into the game, from simple forking paths of early text-based adventure games to the dialogue trees and AI of contemporary games. The videogame event is the relationship between its components (human and nonhuman), its motive is the ludic ambiguity of its capacities and characters. Jo's 
exploration of the geography, physics and pathology of Sandy Bay, and the new little games that emerged, might seem to promise a victory of straightforward human and imaginative agency over the system. But this exploration only stretched the logic of machinic configuration of the player; it did not break it. Jo was testing the game's systems (literally bouncing off the gameworld's limits), and all the while the game was patiently waiting for its prodigal to return to the preferred performance of successfully racing the computer-controlled cars. Emergent play could only open up so much of the gameworld, whereas the rewards - of progression through levels, of spectacular or narrative revelation, of new capabilities and affordances - are reserved by the game for such time as the player decides (or learns) to play the game. That is, we never got beyond Sandy Bay - in the virtual world at least.

Some of the improvized mini-games emerged from a simple assemblage of animated image, and behaviours encoded into avatars and vehicles. Crashing the car and losing bricks repeatedly will eventually leave the player with just the Lego man. The boys found the sight of the Lego man hilarious, running up and down hills as fast as his barely jointed legs would carry him - a kind of real-time, interactive cartoon. ${ }^{8}$ Later, Jo would test the water-death boundary again, nudging the mini-figure deeper and deeper until all but the very top of his baseball cap was below water and he 'drowned'. Even just running the little car-less driver around seemed to resonate with the drowning games and other testing of limits between game life and death:

J: It's funny how he dies, isn't it?

J: I wonder how he dies?

If the concept of 'identification' has any purchase in these boys' imaginative engagement with the gameworld, at best they seemed to slip between a number of 'identifications': being the virtual Lego men whilst playing the computer game; but being the virtual Lego car itself (as we might imagine a child playing with a conventional toy vehicle to be); a hybrid of the two - a virtual car/driver; being the constructors of these men, cars and car-men; being at once the child playing with the actual Lego car-men and coextensive with the car or car-men they are propelling around the room; being the player of a video game and being a meta-player; then deferring the (ultimately passive) role of meta-player to the adult observer and his pretend keyboard. Rather than identification, then, this is a mode of imaginative play that slides around momentary positions of agency, of control and being controlled: a circuitous becoming. When Jo refused 
to write his name on the paper signing-in screen because he was 'the one who makes the Lego Racers go, he was not taking an imaginative position outside the gameworlds, god or king-like, not even the computer itself. He was positioned or disposed by the game event's tranductions of agency of which he was an integral part. He had to be an agent, but not the player (delegated to another human component) or the avatar (he was still driving the car/driver avatar). The game system is the only agent left. An imaginative event was, therefore, configured by a real relationship between agencies and entities. In the virtual game, Jo was playing with (and played by) the whole gameworld/system, in the actual game he was playing as the gameworld system: not one character within a screen fiction, but rather the whole universe that makes the Lego Racers go. He was in the circuit, was the circuit. Imagination was at work, but it is not the familiar imagination of 'let's pretend'. If we can talk of ecosystems of distributed cognition in psychology (Hutchins 1995), then in this event, and in other events involving virtual media, we are seeing distributed imagination.

The events in and around Sandy Bay are tiny instances of what is now a very ordinary and everyday cyberculture. All sorts of genres and imaginaries are built into and played out of video games: military, domestic, cute, fantastical, managerial and so on. Cyberpunk is now just one, and a relatively niche taste at that. Instead, we have the intense and intimate circuits of human and machine played out daily in bedrooms, on mobile phones on the bus and so on. Now the cyborg is mundane, the microethologist needs to be careful not to miss the marvellous within everyday technoculture.

Sandy Bay, in both its virtual and actual aspects, should also remind us that systems, whether ludic or machinic, are never closed. As Gregory Bateson explains, whilst systems such as steam engines, societies and computers can be studied or described as closed circuits, formed from internal 'chains of causation, they are never completely closed off from their wider environments. This 'second order cybernetics' insists that systems are always open. In physical terms, and the laws of thermodynamics, systems must be energized from an external source and lose heat to the outside; but also, and more importantly for understanding the cultural-ecological operations of systems, 'events within the circuit may be influenced from the outside or may influence outside events' (Bateson 1972, 404). The next chapter will explore further the eccentric loops between play, imagination, technologies, media images and bodies, moving out from the virtual gameworld, but still concentrating on the play between the material and the immaterial. 


\section{Notes}

1 University of Edinburgh research project on unsupervised computer joke generation. 'Natalie Haynes's Brave New Algo-World', BBC Radio 4, Wednesday 8th January 2014, http://www.bbc.co.uk/programmes/b03nt9vk.

2 In their multimedia flexibility and ludic affordances, smart phones and mobile devices are toys for older children and adults too (see Lister et al. 2009, 252-253).

3 Though probably not an issue for most WoW players in the age group covered by this book, there have been legal challenges by Blizzard, the game's designers, to the external production of software bots that automate key aspects of the game for players, such as resource-gathering and fighting. These are games that can play themselves, http://news.bbc.co.uk/1/hi/7314353.stm.

4 Championship Manager is not the only game that aestheticizes the spreadsheet. 'SimCity 4 was literally prototyped in Excel. There were no graphics - it was just a bunch of numbers - but you could type a code that represented a particular type of building and the formulae built into the spreadsheet would then decide how much power it had and how many people would live there'. Interview with SimCity lead designer Stone Librande, http://v-e-n-u-e.com/Sim-City-An-Interview-with-StoneLibrande. Accessed 12 February 2014.

5 Often written into the game code to allow the designers to be able to test the game whilst under construction, 'cheats' have been a fundamental aspect of game culture since the first home computers (Haddon 1994, Consalvo 2007, Kücklich 2008).

6 The animation of toys, objects and environments seems intrinsic to the psychic dimensions of children's culture from the magical living objects of fairy stories and the animation of toys in play to game avatars and virtual pets. There must be a connection with D.W. Winnicott's theories of transitional objects, 'which must seem to give warmth, or to move, or to have texture, or to do something that seems to show it has vitality or reality of its own' (Winnicott 1974, 7).

7 Or until the entropy that such games have designed into them (precisely to necessitate and prompt human input) brings about the crash of the simulated city, country or ants' nest in a virtual economic and/or environmental catastrophe.

8 For further description of the machinic and aesthetic articulations of Lego video games (in this case, Lego Star Wars), see Giddings and Kennedy 2008. 



\section{Play Grounds: The Material and Immaterial in Play}

Playground games and computer games are structurally similar, then. They both feature rule-governed structures, quantified assets, obstacles and challenges, dynamic 'engines' of play. They can vary from loose improvisation to tight rule structures. Like drama, and indeed any kind of fiction, they involve an imagined world of some kind whose governing principles are understood to be different from those of the 'real' world [...] Both kinds of game provide complex, often impenetrable forms of pleasure which range from the purely ludic - beating the rules of the game - to the representational - the pleasures of mimicry and role-play. (Burn 2013, 124)

25 February 2007. It's the last Sunday of the school half-term holiday, and we're at the Adventure Play Ground (APG) at Windmill Hill City Farm in Bristol. The sky is overcast, and the players and playworkers are chased in and out of the APG building or under play structures by the occasional shower of rain. There are only a handful of other kids there, a few drift in and out, the other diehards are two girls of around 10 and two boys of around twelve. There are three or four playworkers.

Jo, Alex and Sam (aged between seven and nine) are playing Star Wars. The game is primarily conducted through light sabre battles with sticks. The sticks are broken from branches lying around the edge of the APG. At first glance the game would be familiar across generations of boys' play: boisterous free-wheeling arms and sticks, bodies leaping from play equipment and the negotiation of acceptable and unacceptable behaviour. Occasionally details of the Star Wars diegesis or narrative inflect the boys' dramatic pronouncements and performative gestures and actions - evident throughout the play is a flickering of point of reference from the Star Wars films to the Lego Star Wars video games. 
The interpenetration of the actual and the virtual, the material and the intangible, is a central concern throughout this book. This chapter stops to address the actual and the material in play and games through a microethological study of a play event that took place without the immediate presence of any sophisticated technology. Outdoor play with sticks, climbing frames, friends and action and characters inspired by children's media is both pre-digital, in that broadly - the materials and activities of the gameworld are the same as children's gameworlds long before the widespread availability of computer entertainment media, and - as we have seen throughout this book - post-digital in that the game itself is suffused with images and characters from computer games, and is shaped and directed at least in part by the peculiar spaces, conventions and repetitious temporalities of computer games. So, firstly, this chapter develops an ethological attention to the materiality of play and bodies in play (including natural objects, play technologies, human bodies); secondly, it extends this concern for the material to ask how we might conceptualize the very real, but immaterial play elements or bodies (such as mental imagery, media images, conversation and collective or intersubjective imagination) as they come together with the material; and thirdly, it will reflect on how this gameworld can be understood as actual in the era of virtual media - both in terms of its transductions of video games and in the rethinking of play in general, pre- and post-digital, in the light of virtual media. It will pick up on the suggestion at the end of the last chapter - that imagination in play might itself be transducted and distributed across the material and the immaterial.

The microethology of this event of 'free play' will explore the nature of these worlds within worlds, the kinds of spaces or timespaces they generate, their patterns and dynamics and the phantasmagorical realities they are constituted by, or bring into being. The game - or games - was populated by characters, scenarios and technologies from the media universe of Star Wars (and others) in a imaginative engagement with transmedial ecology of children's popular culture. They were also formed from the material environment of the playground and its buildings (play equipment, sticks, office stationery), as these artefacts both initiated play events and were transformed by them.

\section{Talking into being}

The game began with talk, the boys standing on a play structure, a house-like wooden construction with a ramp leading up to it (Figure 6.1). Sam decided he 
will be Luke Skywalker, Jo claimed Anakin as his alter ego. The talk continued for some minutes, sometimes the children seemed to be talking just to themselves, conjuring up the world they want to inhabit, sketching in the environment and agonistic motive for the action to come. The brothers Jo and Alex argued. They have had occasional arguments in the past over Alex's refusal to conform to the accuracy and continuity of the Star Wars transmedia universe of films, and games and television series, and the particular scenarios Jo wishes to inhabit. The controversy was not so intractable as to stop the emergence of the game, though

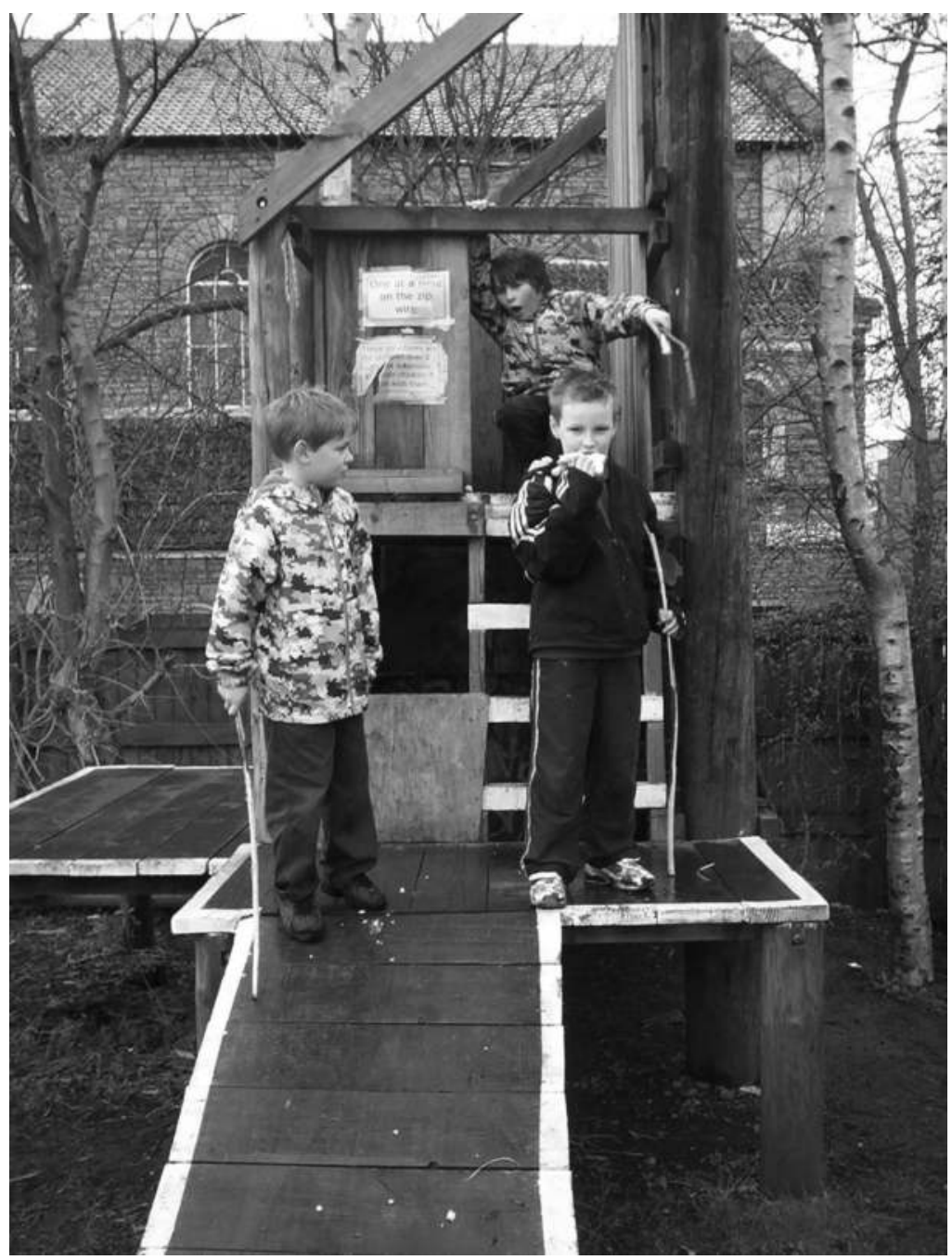

Figure 6.1 Prelude: Talking Star Wars 
over the next half an hour or so it flared up from time to time, temporarily stopping play. I - from my position as interested observer from the sidelines was asked to arbitrate. One such interruption was caused by Alex's dissatisfaction with 'Episode 6', the starting point, and most consistently inhabited scenario in this particular game. The reference here is to The Return of the Jedi, the third film to be produced (in 1983), but with the subsequent 'prequels' becomes in narrative terms the last in a series of six. Interestingly, the children made little distinction in their talk or games between the film itself and its remediation in the Lego Star Wars games that they had also recently been playing. The ending to 'Episode 6' in particular offended Alex:

A (rhetorically):

'What happens to Luke?'

'What happens to the ghosts?'

Alex announces that he is instead going to play 'Episode 7', a storyworld of his own invention. Episode 7 is conjured into existence there and then, but its central protagonist - Starjumper - is a well-established creation of his. Starjumper first made an appearance in our house two or three years before, but had not been heard of for quite some time until this game. This did not settle the dispute over diegesis, however: Jo is very annoyed about this multiplication of playworlds, and loudly accuses Alex of 'cheating'.

Despite the momentary vehemence of this argument the game quickly sprang back to life and circulated around the playground, gathering its forces at key landmarks and particularly useful play structures. It appeared that the imaginary scaffolding of play such as this shifts or mutates according to the shifting modes of play itself: rules, settings, characters, quests must be clearly established as the gameworld is talked and negotiated into being, but once the swirling and flailing game itself is under way the details are superceded by bodily actions and performances that sweep up semiotic and gestural material from other films and games and the play is much more forgiving of continuity errors. The close agreement required in the talking prelude diverged into a kind of imaginative parallelism as the boys held in their imaginations individual iterations of the gameworld, and their dynamic role within it, with the others' actions generically appropriate enough to sustain and develop a looser but more exciting collective imaginative world.

A little later they argued about whether they are on Hoth or another planet, the name of which I didn't catch. Alex is a wampa, a yeti-like creature indigenous to 
the icy planet Hoth in The Empire Strikes Back. This sudden attention to location was triggered, I thought on reflection, by the coincidence of two factors. Up until this point whilst the narrative positioning of the game was of great importance, its geography hadn't been significant, but when Alex took the role of the wampa the distinct climatic character of this monster came to the fore, its white fur inseparable from its snowy environment. At around this time in the game a shower of light rain started. In a simple but powerful procedure of semiotic synthesis, the fictional world and the actual environment were fused to create a novel material-semiotic environment: a new (un-named) planet analogous to Hoth but characterized by rain rather than snow. Alex soon joined in with the energetic wielding of sticks in a game of light sabre combat. These stick-wielding performances are kinaesthetically sophisticated, but their symbolic consistency less so, and wampadom was forgotten, and the new planet disappeared as if it had never existed.

The younger Star Wars boys paid little attention, as they were engrossed in their light sabre driven play (Figure 6.2). They broke off now and then, the talk often returning to the Lego Star Wars game.

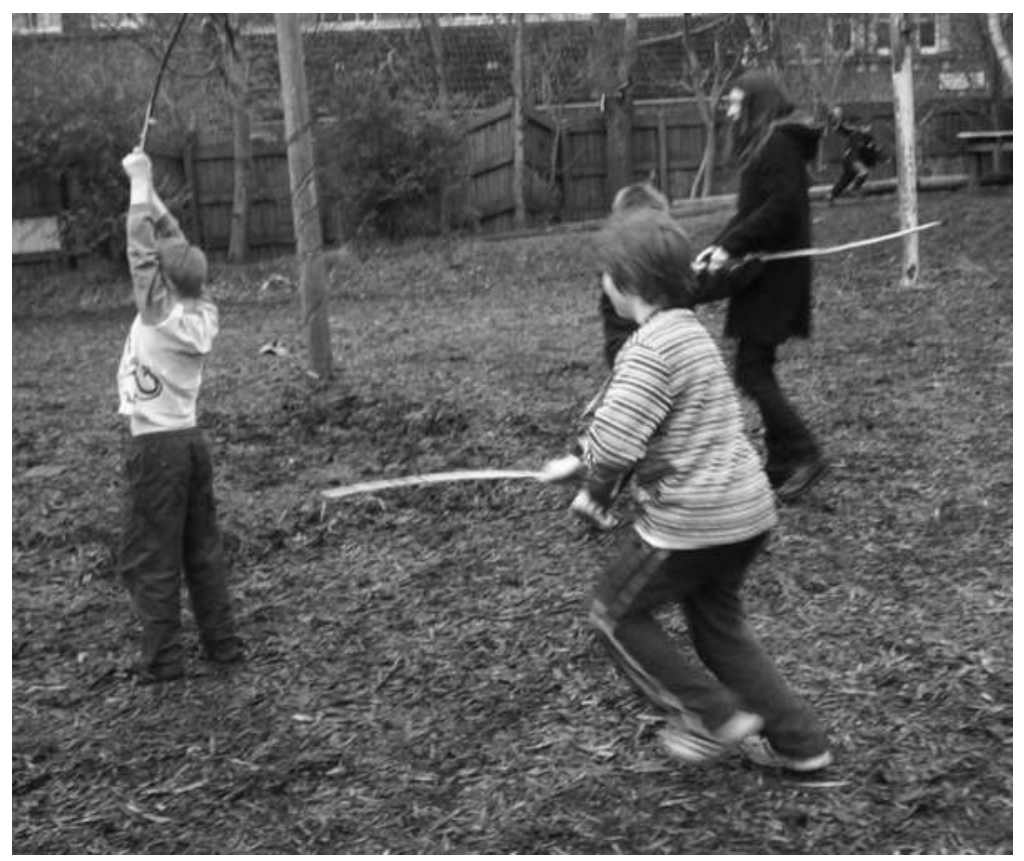

Figure 6.2 Sticks, swords and sabres 
J: 'in one of them if you get a full minikit you get a ghost - an Obi Wan or Yoda ghost'.

Sam had decided that an adult should be included in the stick battles, and while he waited for a reluctant playworker to join them, handed me a stick and I too became a light sabre wielding character. Sam, who had a marked taste for the nonsensical and had affected to have forgotten my name (he has known me most of his life), had already named me George Washington, so I was not allocated a proper character (perhaps because I was merely a stand-in). This shift in number of players from three to four suggested a team-based dynamic as Jo and I take on Sam and Alex. There was some narrative logic to this pairing though in the resulting melee of both bodies and identifications I did not grasp it. I think at one point both Anakin Skywalker and Darth Vader were involved which should have offended Jo's sense of diegetic accuracy but which seemed to pass unnoticed in the swirling action of this particular game-mode.

Apart from this brief involvement in the main drive of the play event I kept to the sidelines, or more precisely a small wooden shelter where I could sit and read out of the rain. My kids had been inside a lot over the preceding week's holiday and had seen a great deal of me so were not as enthusiastic about my involvement as Sam was. I did play some roles, though, mainly referee between my two sons' periodic sulks and fallings-out, but I was also an intermittent audience for Sam's nonsense performance, including an accomplished rendition of the theme to the Mel Brooks' film Robin Hood: Men in Tights. I also helped to make new light sabres, breaking off lengths of branch from the pile of branches near my shelter. The wood was new and green so took some bending and splitting before it could be separated. My stronger fingernails helped to strip bark from one end to accentuate a bright (green) light sabre appearance.

\section{The environment of the playground}

Game spaces are fashioned from the material characteristics and features of the environment, as well as from imaginative and cognitive operations. As described in the last chapter, video game worlds suggest ways of rethinking actual environments (and vice versa) - as space and time, as lawful or rule-bound, as affordances and constraints, as material and immaterial. There is a persistent view, evident from Romantic poetry and painting to today's dire predictions for 
children's technoculture, that the child is truly at play (only truly a child) when he or she is immersed in the natural world. 'Natural' here means the countryside, plant and animal life, the weather:

In the creative perceptions of poet and child we are close to the biology of thought itself - close, in fact, to the ecology of imagination, in which the energies of the body and mind as a unit, and ecosystem, and the energies of nature combine in a mutual endeavour to adapt to nature, to culture, and to the societies devised by man to everybody culture. (Cobb 1977, 109)

Edith Cobb's ecological thinking, influenced by her friends Margaret Mead and Gregory Bateson, does not rule out the artificial objects of children's culture. Her notion of the child's 'cosmo-poetic exploration of the environment' includes plastic play with blocks, paint, 'any amorphous or semi-structured material (e.g. sand, twigs, and stones)' (Cobb 1977, 30). However, the amorphous and semi-structured material is significant here: there is a strong tradition of thought, from the education pioneer Friedrich Froebel in the early nineteenth century to Margaret Lowenfeld's therapeutic gameworlds of sand and water in the twentieth, through to everyday and popular discourses on toys, that toys or other playable fragments of the child's environment should be as semiotically and formally indistinct or open as possible (Lowenfeld 2008 [1935]). The young child's imagination, it is often felt, should not be scaffolded or guided by inbuilt and prescribed stories, characters or shapes (an attitude clearly echoed in critiques of children's media culture outlined in earlier chapters). Just as ethnographic work on children's play with media resources reveals much more fluid and creative events than predicted, though, close studies of play with physical objects and environments, both indoors and outdoors, trace similar complex interrelationships between resources and events. School and park playgrounds offer a wide variety of armatures for play and games. Some of these are intentional - climbing frames, painted courts or hopscotch matrices and so on, others are either improvized for moments of play or established as persistent cultural sites and technologies in their own right, often reproducing games down generations and for decades:

Every feature of the playground is used: the corners and walls of the buildings; the fences (as 'home', or for tying one end of a skipping rope); the ledge outside the largest temporary classroom (for walking along, or as a vantage point, or for a game of King of the Castle); the flat drain covers (as sanctuaries or as marble boards); the small cavities at the foot of 'the marbles fence', where the asphalt 
meets the grit surface of the lane; the dust-bowl at the edge of the grass, used for flinging toy cars. (Opie 1993, 11)

June Factor describes a remarkably persistent material-semiotic culture built into and from the micro-topography of a school playground:

Its inhabitants - children - have developed, sometimes over generations, a map of the school grounds which designates functions and attributes values to every major feature: open space, treed space, benches, shelter-shed, toilets, grass, asphalt, tree roots, secluded corners, verandahs, rubbish bins. (Factor 2004, 143)

A fallen tree becomes a spaceship for generations of boys, its control panels and components shaped from 'the intricate crevices, lumps and nodes caused by the decaying wood' (Russell 1994, 93, in Factor 2004, 147). Whilst two girls, on a public thoroughfare with trees, leaves, log fences played 'princesses and flying unicorns':

The girls used the physical and natural features of their chosen play-site to represent their home and other far-away lands they travelled to... [one girl's] bed was a low pine fence, her shop was a pile of stones, and the kitchen a clump of bushes with a strategically located sawed-off branch which served as the controls for the oven. (Russell 1994, in Factor 2004, 147-148)

There is a two-way flow between environment and children. Through an ecological feedback loop, the former seems animated, like a video game world, attracting children to its playful affordances:

Objects often call out for the young child's attention and exploration: the grass must be run through and rolled in, the sand and earth should be dug up, beakers need to be filled with water and then emptied again although, curiously, the toilet does not necessarily call out to be peed in. This engagement with the world is an interlocution, a dialogue - an object calls out to the child and the child answers. (Aitken and Herman 1997, 83)

An object can call out to the child, and that call can triangulate with a fantastical idea from the imagination or media source in play. For example, a skipping rope in a superheroes game discussed in the next chapter was picked up and used by one of the boys, Henry, for just 'skipping', a long-established and flexible play practice with its own physical demands and expertise. Yet this familiarity itself seemed to form the nucleus of new games, attracting the thematic symbolic elements floating around from other recent and potential games. It quickly became 'really fast skipping' for Henry, a superpower inspired by Dash from The Incredibles (Richards 2013, 77). 
Experience in childhood is never formal or abstract. Even the world of nature is not a 'scene', or even a landscape [...] the child's world, his surroundings, are not separated into nature and artefact. This environment consists of the information fed back to his own body by environmental stimuli. This responsiveness includes all levels of the child as a functioning organism. (Cobb 1977, 28-29)

The bulk of Cobb's observations of play predate children's media culture, but her conceptualization of play as environmental, informational and responsive offers a suggestive model for describing the artificial as well natural domains of the child's world (though I'm not sure she would have seen it this way).

Here is an example of the way in which the material gameworld, and particularly the embodied behaviours of the players, can be shaped by the immaterial forms of video games in particular. As we saw in the last chapter, video games insist that certain cognitive functions and imaginative processes are delegated to their subroutines. They keep score, note location and orientation and enforce death. If a Lego man is submerged or a soldier is shot the gameworld notes it, and responds accordingly - the avatar disappears and respawns elsewhere. When transducted into the less precise material bodies and spaces of an actual playground, new negotiations must be made, and new ways of playing devised:

Andrew: What would happen if you shot someone else and they got killed, then, in that game, what would they do, would they fall over?

Martin: No, they'd have to bob down like that (crouches down) but none of my friends agree that they'd got shot, so you go round prrrrtttt (mimes shooting again).

Andrew: So no one would agree to be dead?

Martin: No, but in the [computer] game, you fall down, the person falls down when they do it, and then has five seconds, and then gets back up. (Burn 2013, 130-131)

The children have to devise a performative surrogation of the non-negotiable registration of game death. Free from software control, other players refuse to imagine their own fatality and the rhythm of the game breaks down. I don't remember this problem in the shooting games in my own childhood; it seems that having been delegated to the computer game's functional imagination, the return of this gestural-semiotic game mechanic to the actual playground and human imagination is something of a disappointment: 
Because physical play cannot reproduce the programmed certainty of this ludic system, it falls back on mimicry (bobbing the head), ineffectually supported by an agreement that this will be the consequence of being shot. (Burn 2013, 131)

The delegation of game rules, frames and other aspects to software is a complicated but significant shifting of the circuits of agency in play. As I have argued, the actual environments and objects of play, from the manhole cover in the playground to the articulate and articulated smart toy, have always suggested, triggered, shaped and sustained games and imaginative behaviour. In the previous chapter, I noted the idea that the rules of a game are often embedded in the gameworld as 'laws', analogous to the physical laws of actual play. Gravity imposes the law that 'flying' players must stay on the ground, or leave it only briefly in a jump or with assistance from the simple technics of a swing or climbing frame. A player, in the intensity of the moment of as-if flight, may feel themselves almost flying - virtually flying - and this must be the game as embodied experience as well as aesthetic or performative form.

Caillois observes that many actual games do not imply rules. The performance of cops and robbers, for instance, or the technically enhanced make-believe of dolls houses 'presuppose free improvisation'. They involve playing roles, 'as if one were someone or something else'. This fiction - the as if itself - replaces rules:

Rules themselves create fictions by the very fact of complying with their respective rules, is separated from real life where there is no activity that literally corresponds to any of these games [they] are played for real. As if is not necessary. (Caillois 1962, 8)

Replace cops and robbers and dolls houses with their digital descendants Grand Theft Auto and The Sims, and the complex circuits between rules, laws and the as-if are unplugged and reconnected. Jo and Alex recently showed me a carnivalesque little mini-game they had devised in a break from the hard work of conducting crime in Grand Theft Auto. Through game settings or a cheat, they turned down the virtual gravity as one might turn down the volume on a television. Rather than trotting through the virtual city as normal, the gangster avatar now leapt ludicrously high above the streets, twisting and writhing, before crashing down and leaping up again. He looked to me like an animated version of Robert Longo's life-size drawings of business men and women suspended, ecstatic, in mid air. The virtuality of as-if flight is transducted into the technological virtuality of the game system; the imaginative operation is 
transformed and split - partly into the playful manipulation of the software (the tweaking of virtual gravity) and partly delegated to the software itself (its machinic enactment of a flight that is no longer impossible, just not the gameworld's default option). A similar logic can be applied to The Sims: the child no longer directly animates the dolls in their as-if aliveness, the software does that. A degree of imaginative control is ceded to the prosthetic imagination. Thus the intangibility of children's imagination is not only laid over inert but compelling material, it is also delegated to machinic analogues. This process by no means replaces human imagination, as the critics of digital play might have it; it extends and augments it - rendering it poorer in some aspects but opening all sorts of new games and meta-games (as we'll see in the next chapter, 'Real Worlds').

\section{The space-time of play}

Like video games, time is a key dimension in actual play. The simple fact of duration, child psychologist Donald Winnicott argued, renders it real: 'playing is an experience, always a creative experience, and it is an experience in the spacetime continuum, a basic form of living' (Winnicott 1974, 67). The stopwatch punches the intense and formalized activity of competitive sport into precise periods. Imaginative play warps time and space into polyrhythms of frenetic and languid activity, and is characterized by repetitions and circularities as much as by the linear continuities of quests and stories. These rhythms are set by immaterial factors such as degrees of agreement, resonance of imaginary framings between players and by material factors from the regulation by school bells of playtimes to the energy levels of the children themselves. Lili Peller explains that in dramatic play

there are frequent interludes in which the ideational content runs low or gets confused and hazy and only the pleasure in some kind of manipulation or repetition keeps the children going. (Peller 1971 [1952], 122)

Moreover, adult attention - whether family or academic - tends to notice play as a sequence of more or less coherent imaginative or competitive games, and not the flows between games nor their repetitions and returns:

Written records of children's family and households play have a tendency to gloss over its incoherences and sudden shifts [...] Yet the play of children under five usually resembles less a stage play more a dream. There are duplications 
of persons and episodes, sudden changes of locality - all of which just don't make sense, not even to the observer who knows the players well. It's amazing how children can apparently enjoy playing 'together' for a long time, their ideas clicking for a while - then go far apart. (Peller 1971 [1952], 122)

For all its shifts of rhythm and mutating media frame of reference, there was a certain continuity and flow to the Star Wars game. At moments, though, it was punctuated by what I can only refer to as equivalent to a video game 'mini-game'. At some subtle signal or cue the three boys ran across the APG to the far corner and an arrangement of green netting strung between some small trees. Once climbed-into, the webbing took on a hammock-like form and the children seemed at first to be having a rest from the frenetic activity of the game-event. Yet these interludes (this activity was repeated three or four times), these gaps in play, are themselves games, suggested by the characteristics of the assemblage of boys, webbing, the shapes and dimensions of movement the boys-in-webbing formed, and generating their own symbolic points of reference. Initially they were in hammocks. Alex was particularly enthusiastic about this as he was always excited by pirates, and he rocked from side to side contentedly. The others climbed in and the hammockness diminished as the tangle of bodies disrupted both the rhythm and the form. To me on the outside it had mutated into a bean pod - an idea which amused the boys who adopted bean-ness enthusiastically. However, probably due to the kinaesthetic and dramatic limitations of this as a game, it lasted only a few seconds. It quickly became clear that the green webbing/tree assemblage is not an optimum play mechanism for three children. Muddy shoes are too close to other's heads and the combined weight results in the middle bean bumping on the ground. With two boys in the pod, however, the hammock and pirates returned, this time driven by the third boy's rocking of the webbing, a motion loudly interpreted by the general assemblage as analogous to pirates trying to sleep in hammocks in a stormy sea (Figure 6.3).

The Adventure Play Ground is a space set aside and designed for play. The children present had their own temporal boundaries, set by parental expectations, meal times and so on. Within this encompassing space and time, though, the playing itself demonstrated a resistance to any simple mapping or schedule. In spatial terms, the games seem to coalesce around particular locations or structures rather than draw a touchline or magic circle around themselves.

There were delimited zones within the APG within which the sabreplay was manifested, areas with enough elbow room for the flailing sticks. Material 


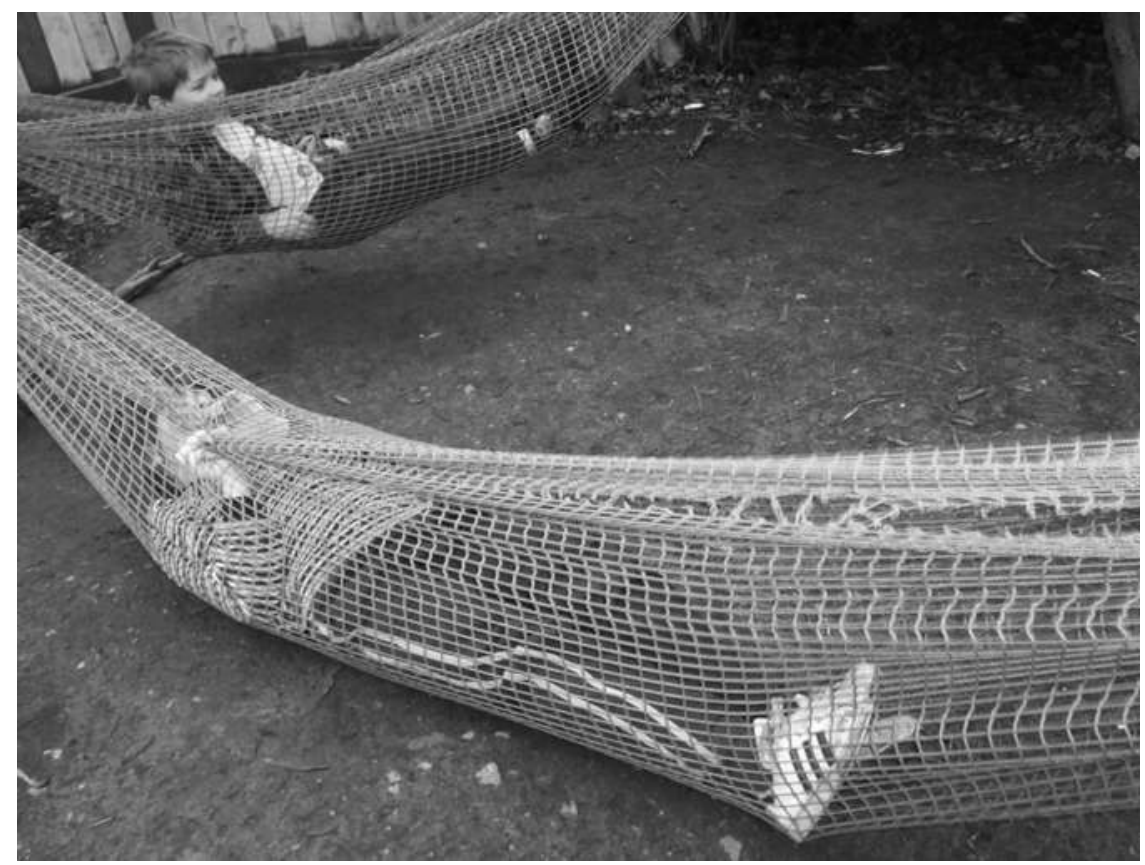

Figure 6.3 Hammocks and bean pods

structures with particularly rich symbolic possibilities (the webbing), but which also set in train temporal and kinaesthetic rhythms of iterative games and swaying hammocks. The players' bodies themselves were zoned according to which parts (including their stick extensions) were acceptable to strike. There was a temporal dimension to this zoning in which initially tacitly (though clearly) demarcated zones are progressively approached and breached: a giddy transgression.

The games had no spatio-temporal boundaries, then, but rather a gravitational pull - either a physical structure (a wooden boat, a webbing hammock) or an intensity of imaginative or kinaesthetic activity (the talking of the game, the swirling of the sabre fights). There were no centres of gravity as such, the games shifted and overlapped too much, but there was a kind of centripetal force that gave some durational and symbolic cohesion - eccentric orbits around an idea or an action. The best analogy I can come up with is that of a skateboarder or BMX rider in a skate park. The circling up and around a bowl is formed by the interaction of momentum, skill, concrete topography and gravity. Movement is fluid and improvized but contained and tropic, always looping back towards, but not necessarily reaching, the centre of the bowl. However, when skaters - by 
accident or design - achieve a trajectory or velocity that ejects them from the bowl they don't exit playspace, they take flight into the neighbouring bowl another centre-less centre of gravity. The boys' sudden arcs out of the Star Wars battles and over to the webbing hammocks, and back again was for me the result of this interplay between the pull of a game (around a particularly compelling fantasy or exciting physical activity) and a centrifugal force (of a competing idea, boredom, distraction) that effects a phase transition.

Just as there are no spatial boundaries, the start and end of these loose games, then, are not signalled by a whistle or even the clear formality of the long-established counting-out techniques of playground games ('eeny meeny miney mo') or the winning state. Observers of children's play and games have noted the transitions into and between games in space-time as well as their structures, rules and rhymes. This account by Iona Opie beautifully captures the rolling individuation of a game from inchoate behaviour:

We strolled over to where a game was brewing. Six or seven children were concentrating on each other, becoming active, becoming a self-reacting entity. Their faces were animated, they communicated with quick smiles. They started running in different directions. One of them shouted, 'Who's on it?' and another replied, 'Helen's on it.' 'I'm no-ot,' shouted Helen. The confusion about who was chaser made the game more fun: muddle is in itself intoxicating, and they laughed immoderately. A boy, meeting them head-on, was brought into the game. He ran away; then realizing he had run beyond the boundaries of the game, ran back towards the others. 'Who's supposed to be on it now?' they called to each other, giggling. 'I think it's Nicky'. (Opie 1993, 84-85) ${ }^{1}$

The game swept up the boy, but his own momentum nearly ejected him from it immediately before he realized the boundary - relative to the movement and intensity and not to actual space - and looped back in.

Actual play spaces (and times) may be 'pure' as Caillois asserts, but they are not homogenous, nor topographic. Finite but without boundaries, they spread their map over the heterogeneous territories of the physical and media environments from Hoth to the Spanish Main.

\section{Bodies}

Back in the stick-fighting arena, a playworker braved the drizzle and joined the fray. She decided to be Darth Maul, a choice that was no doubt suggested 
by a strange little mini-game that will be detailed below. As has been noted, the early insistence on diegetic accuracy and concomitant close identification with specific characters that may characterize the beginning of a game will often evaporate as it shifts more into the material realm of the environment, bodies and kinaesthetic action. The main motive and activity was now the stick battles. These were conducted in the form of sword fights in films in which the alternate angled blocking of the other's sword is performed rather than any serious attempt to make body contact through thrusting or stabbing motions. My slip here from 'light sabre' to 'sword' is intentional as it follows the children's own performances (much more swashbuckling than the martial arts-influenced Star Wars fights) and speech (they slip into talking about sword fights too). The material characteristics and affordances of physical sticks lend themselves much more to cinematic sword-fighting than they do to the fantasy technologies and techniques of the light sabre. No doubt this is another example of playful collapsing of time and space as the momentary pirate world of the green webbing was looped into the overdetermined swordplay. And as I wrote up my notes I realized that Sam's rendition of Robin Hood: Men in Tights must of course have been triggered by that film's scenes of sword-fighting. So much of children's knowledge of literary, cinematic and other cultural narratives, themes and characters comes to them filtered and ludically transducted through parodies and comedy, from Halloween's festive flattening of the gothic to the voracious parodic machine of The Simpsons.

The patterns of play emerged from this risky coming together of bodies and weapons. A distinct rhythm was established: an initial and tentative clicking of sticks by opponents, carefully angled and alternating in a pattern familiar from action film swordfights. This careful alternation would increase in tempo as the fighters' confidence grew, faster and faster until the respectful turn-taking degenerated into flailing arms and sticks, and - inevitably - the contact of stick and body. Knuckles and faces struck accidentally, and backs and bottoms deliberately; these collisions punctuate the full-on battles as the injured player withdraws and the overexcited assailant stops briefly, 'calming down' (a little). The duration of the interlude would depend on the severity of the injury and the degree of outrage of the injured player. Then, the fight would resume, slowly, carefully... The gradual positive feedback as the clashing sticks are wielded more and more frenziedly is sharply regulated by the event of the minor injury, before the fights begin their spiral from simulated to actual pain again. Players' bodies - as borderline accidental targets for stick 
blows, and as demonstrative, media-choreographed, dramatic dimensions or vectors in the game's manifold - are more or less precisely, differentially and emergently, mapped and hierarchized. Lines are drawn in space and across bodies to be - momentarily, intentionally, tentatively, deliciously even transgressed.

After some time, the cluster and clatter of sticks ran low on energy. It seemed that there had been little elbow room for diegetic drama and that this was needed to re-energize play. With no apparent sign, instruction or individual initiative, the pairs split apart in the central arena of the APG, one heading towards a tower supporting a zip wire, the other towards a small wooden boat in its incongruous dry dock. Immediately this generated a geodramatic structure of opposing bases to be stormed and defended. Jo mapped this new time-space onto another of his Star Wars video games, Star Wars Battlefront II, which is based on a well-established videogame motive in which play is driven by the capture and defence of an opposing team/army's command posts.

\section{A micro-carnival}

Two older boys who have been maintaining an aloof distance from the younger children's frenetic activities have found a roll of red circular sticky labels in the play centre office. They are absent-mindedly sticking them onto their hooded tops, first one or two on their chests like badges, then down their sleeves in a regular polka dot pattern, on their hands and faces, and then a playworker and the two girls join in to completely cover the boys' faces with labels. Throughout, the key participants remain serious looking, refusing to acknowledge verbally or performatively what is clearly a ludicrous activity. I hear the phrase 'phantom of the opera' from the playworker as she fashions this stationery mask, though I am sure that the boys' frightening bright-red faces under their hoods resonate with the surrounding Star Wars worlds - they gather to themselves the fearsome and other-worldly gravitas of Darth Maul, without stooping to childish role play (Figure 6.4). For all their studied cool, however, this is for them a significant event as, still deadpan and mute, they wander off out of the APG, no doubt to alarm with their freakish transformation elsewhere in the City Farm and surrounding streets. This 


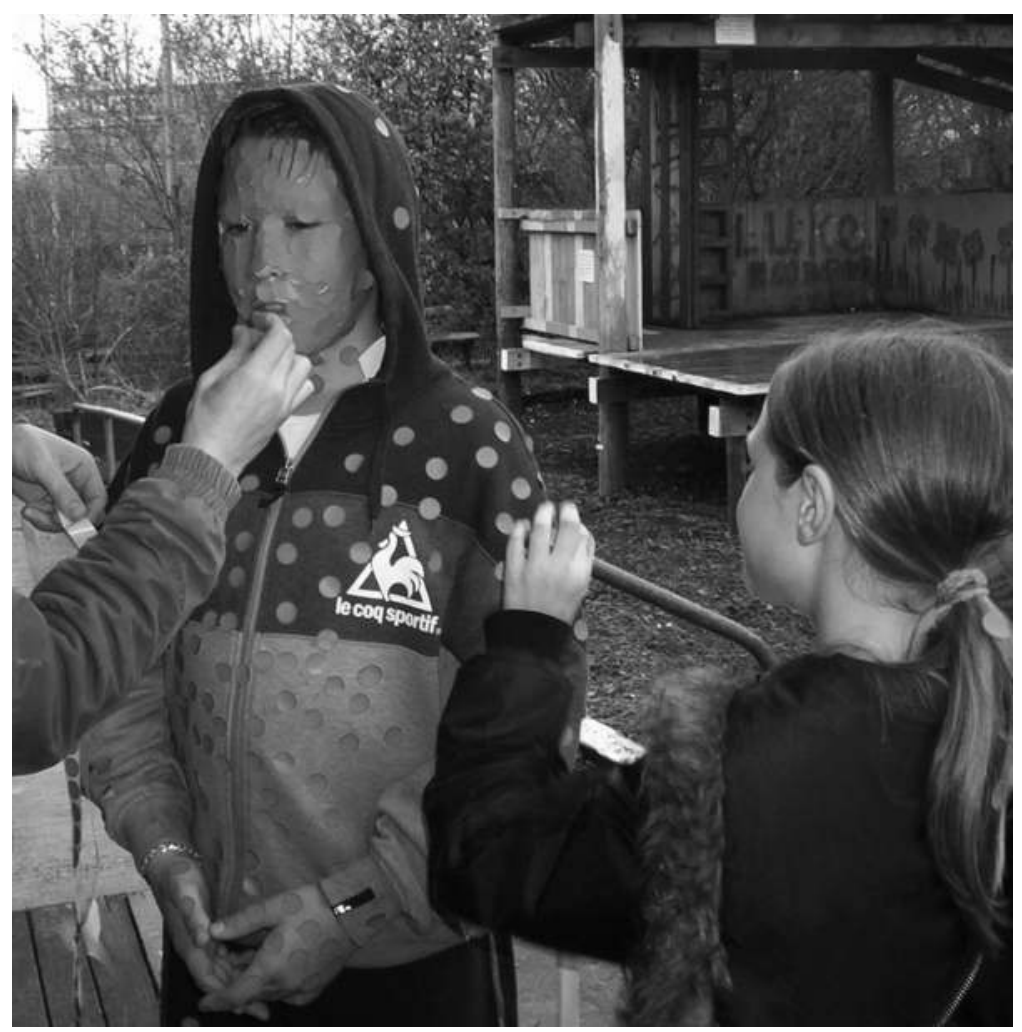

Figure 6.4 Darth Maul stickers

was on the one hand an improvized, liminoid and tiny instantiation of the carnivalesque, and on the other fully part of the logic of children's role play:

It is not enough only to establish an identity for one's self; it must be established for others at the same time. Identities are announced by those who appropriate them and placed by others. Identities must always be validated in this manner to have reality in social interaction. Usually such announcements are silent, accomplished by clothing, the posturing of the body, painting of the face, sculpting of the hair, the manipulation of props, or the physical location of the self on the scene of action. For these reasons, child's play demands costume and body control, and it is facilitated by props and equipment (toys) appropriate to the drama. (Herron and Sutton-Smith 1971, 12)

The phantasmagorical nature of play, evident in all of the overlapping and nested gameworlds described here, will be examined in detail in the next chapter. 


\section{Flux of games}

The flowing of play through negotiated storyworlds, kinetic and risky stick fights, periodic and iterative retreats and spin-off performances would later be summarized as 'playing Star Wars'. My notes and photographs traced a web of material-semiotic metamorphic micro-events - the slippage between Star Wars light sabres and more generic sword fight echoed in a song to a film that includes swordplay, as do any number of the pirate-related artefacts from children's culture that captured Alex's imagination from cartoons and dressing up clothes/toys, to a stage production of Treasure Island he had been taken to a few months before. The peapod/pirate game was both an iterative gameworld and one that emerged, as if through some hyperspace or ghostly visitation, in the sabre/sword melee, the swords/sabres summoned pirates and the wider media trope of spectacular cinematic swordplay. This non-linear dynamic of affect across time and space no doubt ran through the stickered-up double Darth Maul performance and the playworker-Darth Maul's spirited engagement. Darth Maul himself was distributed over a pair of boys with stickers and a playworker with a stick. Swords, sabres and light sabres, or perhaps more accurately, the performance and kinaesthesia of dramatic swordplay, twisted and swung like the webbing throughout the general field of play that afternoon.

Against the formalist notion of games as clearly delineated structures and activities, defined by in part by their end points and winning states, this afternoon reminded me of Deleuze and Guattari's adoption of Bateson's notion of the 'plateau' as a

Vibrant and continuous area of intensities that develops by avoiding every orientation towards a culminating point or external end. (Deleuze and Guattari 1987, 24)

Where is the Star Wars universe on these plateaus? The 'as if' framing of the collective pretend games sketched out a fluid symbolic and physical assemblage, and not a coherent and consistent imaginary world. The game and its mini-games, effortlessly mutated, was abandoned, and returned to. Through the process of being talked into existence, and then in the very different dynamic of freewheeling physical play, the adherence to particular video game or film episodes and characters were condensed and transposed, at times cycled through like a video game player flicking through the inventory for the appropriate weapon or tool for the challenge at hand. Star Wars itself at times fades from the talk 
and gestures, the stick light sabres become swords, pirates come and go and two Darth Mauls manifest in three bodies.

This was no paracosm - if I hadn't had a pencil and paper there would be no trace of its vibrant intensities apart from the stripped sticks and the empty reels of stickers. The kids would have forgotten it by the next day. In the event itself, it was never only in one child's head. Collective imaginative play is real but intangible and immaterial - it couldn't even be said to exist as neuroelectrical activity, as we might speak of the materiality of virtual space in the magnetic particles of memory storage because it wasn't formed in only one child's mind but across three. It was constituted in the space between them and the playground, finding material form here and there in a stick, a gesture, an exclamation and a plastic net.

\section{Note}

1 See also a video recording in the British Library of a child's explanation of the three games they are playing: 'Star Wars, "the tree one", and Tigers - all at once', and the researchers' description of the play environment:

The area is contoured with mounds resembling low hills and is covered with a slightly shock absorbing material - in this case coloured in greens with blue to represent a river running through the landscape. There are also tree stumps dotted throughout this area. The kind of play shown here involves imaginary conflicts and belongs to a broad spectrum of play involving agonistic scenarios - play exploring conflict, contest, challenge and resolution and including family feuds and disputes, military manoeuvres, superhero battles and even football. The sources for some of the play here include Tae Kwon Do lessons at a local college (and advertised in the school's reception area), stories about big cats (from the classroom), Star Wars ... and a mix of additional generic media sources. In these examples, physical contact is controlled, stylized and causes neither injury nor offence. http://www.bl.uk/learning/ langlit/playground/browseadultview.html $\# \mathrm{~cm}=$ Videos\&gm=Pretend\&id=120551\& id2 $=121262$. 



\section{Real Worlds: Realities, Virtualities and the Protopolitics of Play}

My daughter knew that Brownie was only a 'pretend mouse' - this was how she used to refer to him. One day she spilt a glass of milk and accused Brownie. To my 'but I thought he was pretend!', she told me earnestly that 'he's getting realer and realer'. He was her companion for about four years. ${ }^{1}$

Make-believe is 'accompanied by a special awareness of a second reality or of a free unreality, as against real life'. (Caillois 1962, 9)

Play has always prompted reflection on the tissue boundary between reality and fantasy, and virtual media bring new technological realms and animate entities to the layering or multiplication of everyday realities. This chapter will pick up the theme of virtual and actual realities that have been addressed in different ways in the earlier chapters. Throughout this book, I have preferred to use the term as-if, to refer to the generation of moments and events of imaginative play, rather than the ostensibly synonymous 'let's pretend' or 'make-believe'. I want to keep hold of the sense of play and games as real that runs through the most astute and productive theories and descriptions of children's play and culture that I have drawn on here. Whether material or immaterial, embodied in equipment and machines or imagined, mundane reality is transformed or multiplied in play. The opening gambit of the as-if, the approaching or becoming real of the virtual, Caillois's 'second reality' are all - like VR - accessed through technologies and techniques and experienced in bodies (not only human) as real. This chapter will offer approaches to describing the significance of the multiple and shifting realities of everyday play for understanding children's culture today. It is particularly interested in the immaterial or intangible in play and everyday life: the operations, effects, affects and behaviours of software code and entities, and virtual physics, but also dreams, fantasies, collective and individual imaginations. 
My concern throughout is with imaginative play events, particularly those in and around video games and other playful virtual media. These are a significant aspect of contemporary childhood in the developed world, but they are only one part. These gameworlds come into being in the networks of commercial children's media and Internet media, within domestic and educational environments and discourses that are themselves shaped by the power relationships of families, schools, and cut across by social stratifications of gender, class and ethnicity. There is an ongoing, if intermittent, politics of children's culture that addresses the representational and material resources for play available to children, generally concerned about the rigid gendering of toys and popular media, and concomitant questions of the resources for identity formation and aspiration as children move towards adult life. In recent years, for instance, there have been widespread concerns that consumer capitalism has been encroaching on childhood by thrusting adult, and hyper-gendered, behaviours into children's lives and play, with the marketing of make-up and sexualized clothing for 'tween' girls or the violent, military themes of video games with adult ratings but played extensively by younger boys.

Children's play today worms its way through the rigid gendering of toys and narrative media and the commercial imagineering of transmedia and online spaces. However, as I argued in Chapter 4, research that assumes or reads off playful behaviour from these powerful political and economic realities and their materials, media and environments may miss the peculiar imaginative and physical inversions and fantastical operations of children in play. If a critical concern with children's culture begins and ends with a critique of the mimetic dimension of the media and toy resources available, then the concern could be counterproductive. In this book, I have tried to work from the momentary events of gameworlds and playgrounds (virtual and actual) up. The dervish circuits of imaginative play whip up physical and semiotic material, invert the natural and cultural order so that torture is a delight, objects come to life and individuals become multiple. The profound ambiguities of play seem to subvert and parody, but games are also played by the rules.

Gameworlds are heterotopian, not utopian. Rules are played with and broken as well as obeyed. Children test the roles they see ahead of them, the sexualities they are beginning to be aware of. The hyper-gendered bodies of muscular superheroes and wrestlers or Barbie and the Bratz are compelling in an intensity that cannot be explained simply by the power of marketing and peer pressure. The rigid binaries of male and female, reality and fantasy, 
child and adult, individual and collective, human and non-human are simultaneously exaggerated and dismantled in play. Moreover, attention to the events in which media play takes place highlights constraints and affordances of the material environment from playground furniture to more complicated technologies. These connect with and articulate symbolic and narrative material but have their own politics designed in or emergent in particular moments of use. To oversimplify, it is more important that boys and girls have a space that is safe (but not too safe) and open (but with interesting corners) in which to play together or around each other and with useful materials to mobilize and transform than it is to police the semiotic characteristics of these materials.

I will tentatively sketch out what I call a protopolitics of play, in which realities, agencies, activities and passivities and meta-games seem not only to reproduce cultural and social forms, but also to generate new times, spaces and assemblages - new realities. I will explore this protopolitics through five main overlapping concepts, picking up themes and concepts already discussed in this book. Firstly - and briefly - a note resisting the unqualified celebration of children's imaginative play, and the ambiguities of power and transgression in 'bad play'. Secondly, the 'multiplication' of realities, from the real effects of fantasy to the actualization of the virtual, has been a theme throughout, but is essential to an understanding of how intangible phenomena such as the imagination and virtual worlds, and play itself, operate within and transform everyday life. Thirdly, the peculiar tendency of play to render hierarchies, rules and meanings ambiguous or even to invert them - from the momentary nonsense of a joke or song to the medieval carnival's turning of the world upside down. Fourth, but closely linked to the third, I will pick up and apply Brian Sutton-Smith's eighth rhetoric of play: phantasmagoria, and will argue that without attention to it we can know little of the nature of imaginative play, with or without technology, and nothing of play's monstrous potential to reinforce or undermine social norms. And finally, I will address the phenomenon of playful events that are mediated by an implicit or explicit recognition of their own (real) artifice. The 'as-if', 'let's pretend', 'suspension of disbelief' and so on of play and playful media in and of themselves trouble simple models of media ideology. Players may feel their play to be more real than everyday reality, but they also know and feel (perhaps because of this hyperreality) that it is play. I will explore some examples of meta-play and meta-games, which also suggest ways of thinking about play, culture and communication in more general terms. 


\section{Bad play}

Perhaps the type of (gendered) imaginative play that has generated the most anxiety, argument and debate is boys' war play, play with toy guns, knives or other forms of fighting and combat. War toys and war play have been scrutinized and agonized over for decades, and toy guns, or any object or gesture intended to act as a toy gun, have been banned in many schools (see Smith 1994). Research by Burn, Willett and Richards in English playgrounds in the past few years found that even playful gestures indicating an imaginary gun or knife were forbidden. However, boys improvized their own work-around by adopting gestures from the amalgam of martial arts and magic powers that characterize many contemporary animated television programmes.

This policing of playground gestures and actions, of course, cannot be separated from ongoing debates on the effects on children of war films, violent videos and of course military/action video games. These discussions have been particularly fraught in the United States in the aftermath of a number of mass killings in schools. In all these play forms, in different ways, the anxiety or assumption is that children (or some children) cannot tell the difference between play and reality, or may be more likely to adopt violent behaviour in later life.

Critiques of these 'media effects' assumptions are well set out and argued elsewhere. ${ }^{2}$ What a close descriptive attention to play can contribute, I would suggest, is a sense of the complex interplay of fantasy, imagination and fear - or excitement - about real violence in the world.

We are camping, soon after the riots that swept through English cities following a police shooting in August 2011. Jo, Alex, and Alex's friend Niko are playing with some Lego in the tent. I record their talk, and later take photographs of the aftermath of the game (Figure 7.1).

J: I only have two people, but they have sticks!

A \& N: fighting/shooting/impact noises

$\mathrm{N}$ : Is my guy ever going to die?

A: No!

$\mathrm{J}$ : This guy dies.

A: This guy's my last rioter...

N: No! No! Not yet, he doesn't die yet!

$\mathrm{N}$ : I'll tell you when he can die.

J: There's a fake policeman ... Alex, I killed him with his own neon riot stick 

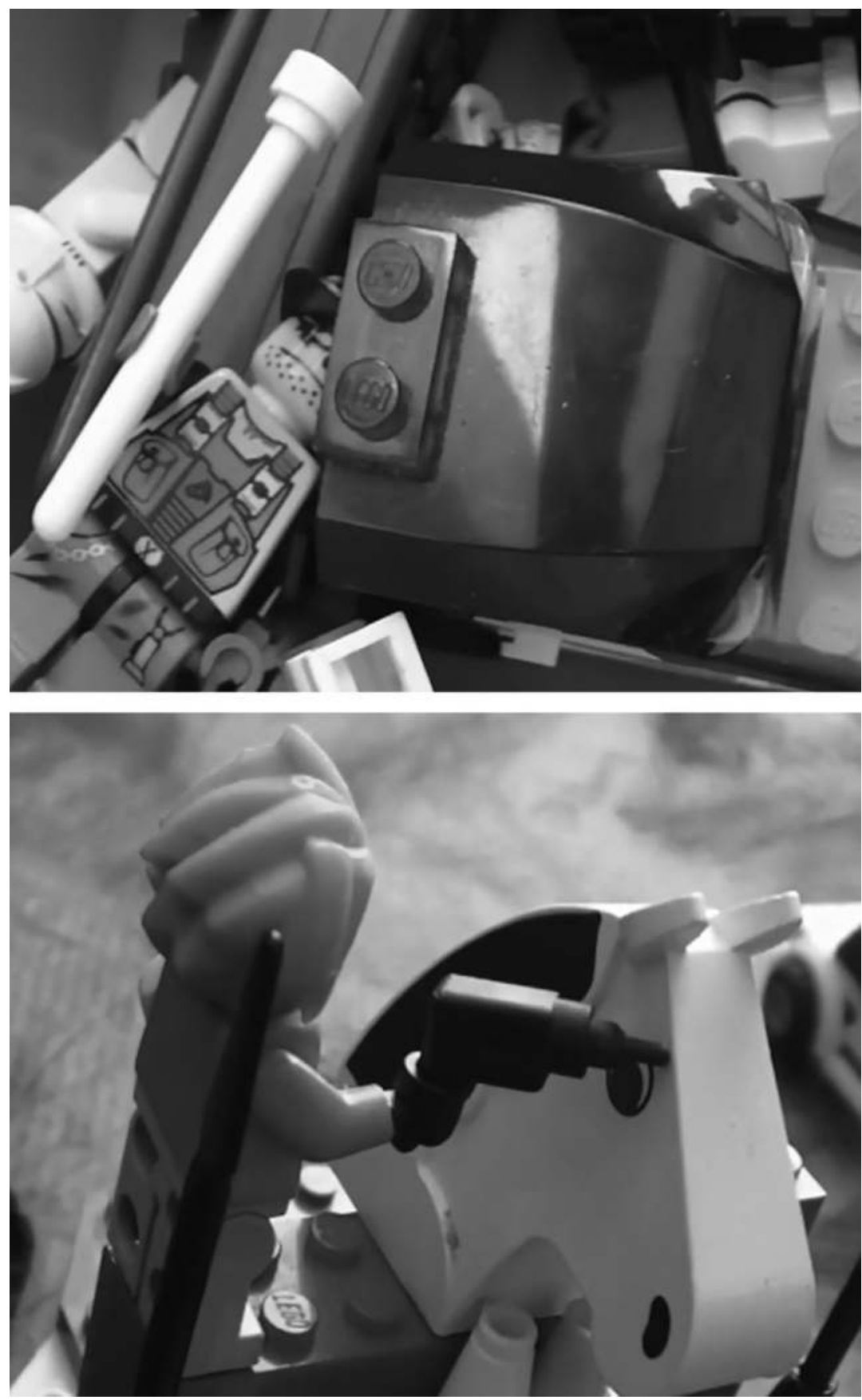

Figure 7.1 Lego riot 
J: You know riot shields? This is what they do...

J: Alex, I'm just beating this guy to death with his own stick!

$\mathrm{N}$ : The police have got a robot!

A: Err! Err! Err! Err!

J: It's a bomb disposal.... I've beaten him so hard his legs fell off.

J: Grr! The last rioter alive!

Whilst it seems to be generally understood that children know the difference between playing violence and actual violence - a fight is an utterly different experience to make-believe combat - for some parents and teachers, the noise and aggression of war play appears to leave no space for boys in particular to exercise their imaginations or develop verbal and emotional relationships. ${ }^{3}$ Hence teachers or playworkers may intervene to channel play into more constructive patterns (see Smith 1994 for a study of the war games and toys debates).

Imaginative, 'free' play isn't necessarily always creative, fulfilling and joyful. It can be repetitive, boring or bullying. Jo's mini-game with the Lego Racers in which he drove the little car repeatedly over a cliff, drowned and respawned shed any obvious imaginative or even pleasurable aspects along with the virtual Lego bricks and seemed to be driven by thanatological feedback between the computer game's cybernetic grip and his own encroaching sleepiness. Iona Opie documents numerous desultory moments of mild cruelty among the playground's exuberance and collusions (Opie 1993). Brian Sutton-Smith notes games between siblings that seem to be shaped by the attractive force of younger children's desire to be involved and the repulsive forces of ludic humiliation, and even harm, from their older brothers and sisters. Often, 'for the younger sibling, the price of fun is getting hurt' (Sutton-Smith 1971, 104).

Scarlett et al. draw attention to what they call 'bad play'. Acknowledging the contentiousness of the term, they are careful to point out that it is not the symbolic material in play that they consider 'bad' (in the sense of contemporary anxieties about make-believe war or sexualized toys), nor rough physical play, but rather play that

does not allow for the socialization process to take place in positive ways for all children involved. Play that excludes or ridicules other children and play that is destructive qualify as bad play. (Scarlett et al. 2005, 80) 
We might note that as with all play boundaries, that between good and bad is shifting and permeable. Other playground ethnographers have observed that the techniques and gestures that signal the start of a game and the constitution of its players may also, more or less subtly, exclude children on the periphery (Richards 2013, 75-76). The younger sibling must balance pain and pleasure; ridicule is a dominant mode of friendly communication for boys well into teenage years and early adulthood; and the borderline between playful destruction and vandalism is one drawn as much by social context as by individual motivation.

Throughout Jo and Alex's childhood in video game culture, the moments of anger, tears and aggression (mainly against the game technology itself) were triggered not by violent scenarios, characters and action in the digital gameworlds, but by frustration with the game on the structural, ludic level. Losing online at FIFA has resulted in Xbox controllers and even mobile phones being broken, whereas the frenetic and ultra-violent online combat of Call of Duty: Modern Warfare 2 seems to generate nothing but hilarity. This topsy-turvy generation of frustration in the breakdown of 'violent' play, rather than in its fantastical performance, is well-illustrated in this account of a play event:

A respected and popular boy in the group is sitting bound to a chair and is being whipped - about 30 times with relatively hard blows - by two other boys with a leather strap. The roles are assigned: He cries out accordingly, 'Ah!' 'Oh!' Two girls give the bound boy some blocks as bananas to eat. The two boys wielding the whip join in and give the bound boy something imaginary to drink. The game thus experiences a resolution through which new excitement may be generated: The whipping proceeds. Obviously, all the playmates are satisfied, the game is, for all intents and purposes, okay. When the teacher intervenes, the children become directly aggressive; chairs are thrown around, and everybody is in a bad mood. (Wegener-Spöhring 1994, 97)

The teacher's alarm at this simulation of torture is understandable but apparently misplaced, for - as the researchers note - the game was relaxed and fun for all the participants, not least the 'victim'. It is the disruption of the game system the intervention of the everyday exercise of benign authority in this case, the cybernetic gameplay in the preceding examples - that triggers stress and actual violence (against objects at least). 


\section{Realities}

A baby doll is not a real baby, but it is not not a baby. (Gregory Bateson, cited by Sutton-Smith 1994, 144)

One of the most dominant rhetorics of play is that of progress or development that children and animals prepare themselves for the reality beyond parental protection through play-hunting, play-evasion and so on. For human children this includes the acting out of the adult world they see ahead of them, playing at doctors or dressing up in adult clothing. As Sutton-Smith points out, 'This belief in play as progress is something that most Westerners cherish, but its relevance to play has been more often assumed than demonstrated' (Sutton-Smith 1997, 9). This notion of play as progress is particularly evident in educational and psychological discourses, but it shares underlying assumptions about the nature of culture and communication, about the mediation of the real with play, with critical approaches to media culture touched on in Chapter 4. Both assume a more or less linear transfer of images and ideas from an exterior world to the interior world of imagination. Children's play is seen as fundamentally imitative or mimetic. Any attention to the transductive operations of play, however, demonstrates that the 'transfer' of non-play elements of reality is rarely if ever so straightforward. From the necessary surrealism of play's magical animation of toys to the misunderstanding or embellishment of actual events (as in the Lego riot), mimesis at the very least has to project its images onto other textures and moving bodies. Though play at first glance may seem imitative or mimetic, often the marvellous is generated as much by the repurposing of available materials available as by the child's imagination. Consider, for example, the scene in the film Toy Story where Hannah, the younger sister of the toy-hacking Sid, comes across the space toy Buzz Lightyear and incorporates him into her dolls' tea party, dresses him up with apron and hat and renames him 'Mrs Nesbit'. Such transductions are probably near-universal in play with toys. ${ }^{4}$

Play may start from a reassuringly imitation of reality (family group, adult jobs, etc.), but has a marked tendency to then rapidly move away from these realities - and not towards them as one might expect if play were primarily 'training' or practice for adult life. Take this transduction of adult family life, for example:

The researcher asked 3 children what they were playing. Each of them said 'families'. In their play, Henry was a brother to a younger sister, Marie. Jack was also a brother, though his position in their age order was not immediately 
specified. Marie commented: 'We act like normal families, except we don't do what families do, we do different jobs'; Jack added: 'We sort of shout at each other. If it's time for someone to go to school you just get go to school and shove them out of the house'. So, given these comments, it was possible to see the bedding down, the restlessness and the brief tussles as enactments of sibling relations. But they were more than that. All three were also secret agents. $X-3$ (X-Men), Spy Kids and The Incredibles were offered as sources. Henry had water powers (like Frozone in The Incredibles). Marie could stretch, 'like Elastigirl'. Jack was the mechanic - 'I just build all the weapons so I just go into my workshop and build something and ten seconds later I come out with a weapon' and 'I'm sort of half Mr Incredible and half Dash, because I'm really strong and I'm really quick'. (Richards 2013, 77)

The children clearly weren't training to be mummies and daddies and gradually edging towards realizing those adult roles. Here instead a familiar domestic setting serves more as a springboard from which to launch into dream-like condensations of film characters, powers, with some scraps of the surface features of adult behaviour caught up in its semiotic and kinetic dynamism.

With its surface familiarity but its animation by more abstract and playful systems of signification, this imaginative play resonates with the structure and processes of the simulation game. A Sims family and home may be constructed along mimetic lines of the player's aspirations or fantasies for future life, but they are driven (as we saw in Chapter 5) by quite different motives and possibilities. I would suggest that as well as using the virtual as a way of rethinking the time and space of play in contemporary technoculture, we might view imaginative play in particular in terms of simulation, not imitation or representation. A simulation, in both its philosophical and computer senses, is a model or copy without original, and simulacra are self-moving devices with behaviours that go beyond that suggested by their surface appearances (Giddings 2007b, 2014c).

Children's play fantasies are not meant only to replicate the world [...] they are meant to fabricate another world that lives alongside the first one and carries on its own kind of life, a life often much more emotionally vivid than mundane reality. (Sutton-Smith 1997, 158)

\section{Poesis}

The computer simulation's predictions, its generation of 'synthetic histories', are productive of reality, then, not merely or directly reflective. In his ethnographic 
research of Huli children's play in Papua New Guinea, anthropologist L.R. Goldman separates imaginative or imaginal processes into two types that support these distinctions in the relationship between play and non-play realities. In terms that he takes from Coleridge, he distinguishes the catoptric and the metoptric (sometimes written as catatropic and metatropic). The former is a mere mirroring of external reality, the latter a 'transposing through rearrangement'. They correspond with the distinctions made throughout this book between media communication as the transmission and reception (more or less as intended) of a message and the playful production of culture through the collusions of play event. In Goldman's terms, they mark the difference between 'reproduction and recreation, the unadorned and the adorned, fidelity and fantasy', establishing a notion of children's fantasy play as poetic and poeitic: 'a dramatic mimesis of human behaviour; a mimesis in the sense not of bland reproduction, but of something transformed' (Goldman 1998, 19). Children's imaginative performances, then, 'are never intended as veridical representations, real-world documentaries. Rather they present as distorted simulacrums, not copies but editorialized caricatures incorporating embellishment and exaggeration...' (Goldman 1998, 19).

The simulacral operations of imaginative play open up a different kind protopolitics to that of 'representation'. Beth Cross, for instance, notes the implications for the expression of ludic or cultural power in metroptic play:

Metatropic mimicry by its very nature is a process open to variable interpretations, and therefore a useful strategy of those in subordinate positions. This may be one of the reasons it is such an attractive tactic for children. It works within the power structures without overtly challenging them. (Cross 2005, 128)

\section{Phantasmagoria}

With the kraken attack, the already unstable paddling pool/Age of Mythology gameworld described in Chapter 1 dissolved in a metroptic cloud of noises, actions and fantastical allusions - like a decaying subatomic particle ejecting exotic new objects:

J and A: Argh! Man overboard! We're going to use the lobster as [indistinct]

$\mathrm{J}$ : But these is related to a crayfish

$\mathrm{J}$ : Here is an underwater dinosaur 
J: and then they boil him, and eat him!

A: (exaggerated munching noises and gestures)

J: He's related to ... what's a worm-like thing that lives in the sea?

A: A seasnake?

A: Yum, yum!

A: I'm a robot!

A: He should just malfunction, and explode

A: He's dead, that guy's dead for good

A: The crayfish is dead! Yummy!

A: That guy should be malfunctioning...

A: Actually, my robot should malfunction and explode as well

Sutton-Smith notes that children's own stories 'portray a world of great flux, anarchy and disaster' often without resolution and with 'repetitive episodic plots', a 'preference for rhyme and alliteration' and characterized by nonsense, obscenity and 'crazy titles, morals, and characters' (Sutton-Smith 1997, 161). He cites an energetic story told by a four-year-old boy:

Once there was a dragon who went poo poo on a house and the house broke then when the house broke the people died and when the people died their bones came out and broke and got together again and turned into a skeleton and then the skeletons came along and scared the people out of the town and then when all the people got scared out of town then skeleton babies were born

and then everyone called it skeleton town

and when they called it skeleton town the people came back and then they got scared away again

and then when they all got scared away again the skeletons died no one came to the town so there was no people in that town ever again. (in Sutton-Smith 1997, 161-162)

Observations of play often reveal either a bricolaged aesthetic, in which fragments of songs, gestures, powers, relationships and so on are accreted in a flow of symbolic and performative activities, or substantially more nonsensical or phantasmagorical mixing up of media tropes, playground games, toys, everyday concerns, rhythms of sound and activity. As with the less rarified realms of cultural expression from medieval carnival to Victorian fairs and freakshows to all the grotesqueries of animated film and television, and, of course, video games, children's culture and play are populated with monsters, ghosts, zombies, 
witches, unicorns, robots and magic (Caillois 1962, 134-135, Klevjer 2006) (Figure 7.2). Like the carnival, children's oral folklore of jokes and songs is also shot through with a fascination for grotesque bodies and bodily functions, in part through hyperbolic or euphemized sexual and scatological allusions:

The bell must have rung because the football came to a halt and the footballers gathered by the classroom steps, still full of uproarious energy. 'Shall I tell you my nicest story?' said Paul, the lanky blond sophisticate. 'There was this lady and a man, and he said, "I'm going to get divorced from you, you don't half smell. You've got rubber lips and smelly breath. The rubber lips is your bottom and the smelly breath's your fart". I made that up'. He got his breath back and recited,

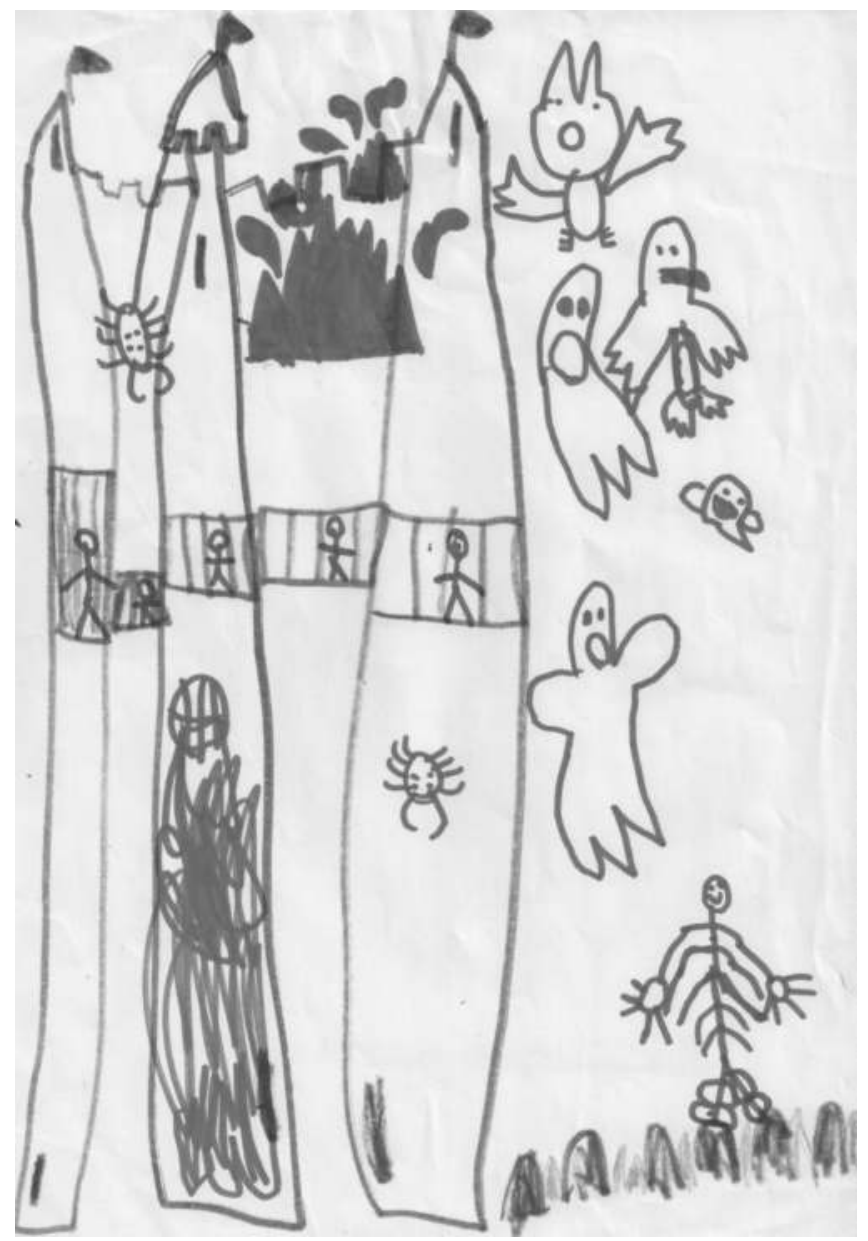

Figure 7.2 Phantasmagoria 
A man's occupation

Is to stick 'is cockeration

Up a lady's ventilation

To increase the population

Of the younger generation.

'I know one', said Andrew.

There was a young man from Cosham,

Who took out his balls to wash 'em.

His wife said, 'Jack,

If you don't put 'em back

I will tread on the buggers and squash 'em'.

The polite version of that, which I have known since the 1930s, concerns eyeballs. I am not sure I don't prefer the rude one. (Opie 1993, 86)

In its irruption of the monstrous and the taboo into everyday life, its synthesis and condensation of heterogeneous symbolic material into an irrational yet integrated pattern, phantasmagorical play has a dream-like quality. Some child psychologists have identified a similar psychic-semiotic structure to play as in Freud's dreamwork:

a play act - like a dream - is a complicated dynamic product of 'manifest' and 'latent' themes, of past experience and present task, of the need to express something and the need to suppress something, of clear representation, symbolic indirection, and radical disguise. (Erikson 1971 [1951], 129)

It seems evident that to some extent traumatic or libidinal material finds expression in play, but the degree to which studies of play could afford the psychoanalysis of players falls outside the scope of this book, and I would also suggest that this return of the repressed seems to be at most just one stream in the turbulent confluence of gameworlds. ${ }^{6}$ The boys playing 'Lego riot' were no doubt in part responding to the disquiet and excitement driven by their emerging awareness of violent events in the wider world into which they are gradually moving. But their carnivalesque invocation of lasers and police brutality, and the surrealist juxtapositions necessitated by the Lego itself (including bodies, technological fragments, robots from Star Wars, etc.), was driven too by the symbolic perversity of play, a depthless orgy of signifiers and materials. As Susan Stewart puts it in her study on the aesthetics of nonsense in literature and folklore, 
Nonsense involves a transgression of common-sense interpretive procedures, a hermeticism in its establishment of another domain of reality, and [...] not a simple rearrangement of the hierarchies of common-sense discourse, but a transgression of such hierarchies. (Stewart 1989, 37)

The affectual hold of nonsense's hermetic domains can be extremely powerful in both its aesthetic and behavioural prescriptions. The rules of the paracosm, for instance, are systematic and absolute (Cohen and MacKeith 1991, 53), whereas for older children, nicknames, graffitti tags, slang, singing and clapping games, hairstyles and fashion, are meaningless in conventional communication, and their rules and syntax change constantly - but at any particular moment they are absolutely precise and key to the sustenence of the micro-worlds of friendship groups and subcultures.

Phantasmagoria is often the result of a simple symbolic algorithm. For example, Jo, Alex and Niko played on an old tractor in the grounds of a National Trust house. They took it in turns to sit at the wheel and drive the virtually careering vehicle. On the way there they had been talking about Grand Theft Auto. They had not been allowed to play the 18 rated game, but were excited about it and may have watched Niko's older brother playing. Actual tractor plus imagined video game equalled an energetic and ludicrous game of Grand Theft Farmer, complete with 'gangster farmers' and drive-by shootings (of other visitors walking past the static tractor - here, literally in another world). Perhaps the simplest phantasmagorical operation, though, is inversion. The medieval carnival turned the world and its hierarchies upside down for a day, with donkeys elected pope, idiots crowned king and men and women wearing each others' clothing. Blasphemy, licentiousness, promiscuity and gluttony were sanctioned for the brief interlude of the feast day. Contemporary Halloween inverts the residual Christian cosmology of the West as a liminoid hell opens and well-behaved children become monstrous, undead, animal and - again - gluttonous. ${ }^{7}$

\section{Meta-play}

Children know that they are manipulating their thoughts about reality, not reality itself, and they know that their play self is not the same as their everyday self. (Sutton-Smith 1997, 159)

The playful nip denotes the bite, but it does not denote what would be denoted by the bite. (Bateson 1972, 180) 
Josie and Lily: I'm a good dancer, dancer, dancer...

I'm the main character, character, character...

Though phantasmagorical play appears dream-like, there are important differences between play and dreams. The other-wordly realms of the paddling pool with its dinosaurs, robots and monsters, or the playground ghosts in a hooped transduction of video games, are structured by ludic demands and conventions as much as by the polymorphous perversity of any individual player.

Lily (excitedly): There's a ghost! There's a ghost on Level Three!

Rebekah: On Level Three there's a ghost? So now how did you find the ghost?

Lily: The thing is, there's a ghost, and we have to catch it -

Sophia: And then they're dead!

Lily: Yeah, but then more ghosts joined, so whoever gets caught by a ghost is a ghost.

Rebekah: Oh I see, and then what happens, if you're a ghost?

Lily: If you get caught as a ghost, then you turn into a zombie...

Sophia: The hula-hoops is ice, and the gaps are, is the world.

The design of the game acquires, then, a degree of representational staging, with ghost and zombie characters assigned, and imaginary spaces delineated. Meanwhile, the ludic structure is expanding into Level Three. The final extract shows, amid even greater excitement, the satisfying mix of ludic structure and suitably horror-themed content:

Rebekah: How was Level Three?

Sophia: Really scary!

Lily: We're on Level Four now with a zombie!

(Burn 2013, 138)

The girls here are playing a game, not dreaming a dream, however they are also playing at playing a game; their game has a (video)game nested within it. Gregory Bateson's insights on meta-communication are useful here. Bateson's observations of animals at play, and his application of these observations to human communication, have influenced game studies in its theorization of the boundaries between playful and non-playful behaviour. However, the full implications of Bateson's ideas are far-reaching. Like Huizinga, he suggests that play in a broad sense is generative of human communication and civilization, not a by-product or epiphenomenon of culture. Whilst Huizinga traces the development of culture as playful through the formalizing and 
cultural-reproductive performances and architecture of ritual, art and law, Bateson's more speculative thinking suggests to me that communication is fundamentally simulational.

He watched two monkeys playing in a zoo. He defined their playing as 'an interactive sequence of which the unit actions or signals were similar to but not the same as combat'. It was clear to both the human visitors and the monkeys themselves that 'the sequence as a whole was not combat' (Bateson 1972, 179). It is important to understand not only that the monkeys knew they weren't fighting, but that they mutually recognized that their activities were 'not fighting', that is what they were doing shared features with fighting, but without the actual aggression and harm. This mutual recognition, Bateson argues, can only be initiated and sustained through meta-communication, that is 'exchanging signals which would carry the message "this is play" ' (Bateson 1972, 179).

In commentary of Bateson's ideas, these observations are often abbreviated to the phrase 'the nip and the bite, where the nip is the playful use of the teeth in the 'not fighting' game, and the bite would be a real aggressive use of the teeth, drawing blood (e.g. Salen and Zimmerman 2003, 371-372). As far as this goes it, on the one hand, demonstrates the necessarily communicative nature of play - to ensure mutual recognition that this is a social and not an aggressive meeting - and, on the other, that there is something representational about play the nip denotes a bite, 'signals standing for other events' (Bateson 1972, 181). Yet this theory of meta-communication has further implications. The metacommunication, he explains, is 'a negative statement containing an implicit negative meta-statement', or to put it in a different way, the statement 'this is play' can be expanded to ' $\mathrm{t}$ ] hese actions in which we now engage do not denote what those actions for which they stand would denote' (Bateson 1972, 180). A dog might bare its teeth or use them to hold or pin down another dog to intimidate it without necessarily fighting or causing injury. In this case the 'nip' would fully denote a bite - it is still not a bite, but the narrowness of the border between representation and actual fighting itself acts as a warning or threat. ${ }^{8}$ In play proper, though, there is an extra remove - the bite itself is absent, it doesn't exist and is understood not to exist (unless the meta-communication breaks down and play ends).

I also speak of this compound of subjunctivity, belief, action, intensity, and commitment as meta-action, because it is an action that is central and stylized, and that creates incongruity through indirect allusion to the mundane 
world ... children are playing with interpreting their own feelings and thoughts, not primarily playing with repeating the external world. Playing is on the meta level, not the mimicry level. (Sutton-Smith 1997, 198)

So, play in its very nature operates on a meta-level of communication. It opens up a gap between the mundane world and its own everyday realities. With children's play, not least play with media forms and images, this gap itself opens up - concertina-like with folded levels of signals and performances. Perceptive ethnographies of children's play pay attention to these levels and mechanisms as children perform media scripts rather than living within, or fully identifying with their fictional world. The singers of the playground renditions of Single Ladies (discussed in Chapter 4) are both performing (to themselves, anyone in the playground who might be interested and to the researcher and camera) and playing at performing. They are learning the dance but also the dance as a media spectacle, inseparable from the aesthetic form of the pop music video. They adapt the dance to fit the demands of playground rather than soundstage performance. Anna, the leader of the Single Ladies performances, for instance, practiced the song not as enactment or dramatization of its lyrics, but as play with the idea and appeal of performance itself:

always I am daydreaming when I listen... when I daydream I think like that like I'm at home and lots of people are at my house to watch me on the stage.

(Willett 2011, 350)

Through media culture, then, the framings of meta-play often include the remediation of music videos, films and television programme. Willett also describes a group of boys playing the quiz show Who Wants to be a Millionaire with questions from an official book, and with performances closely matching the overall programme format. The game is as much about the conventions and iterative structure of the quiz show and the performance of the host and contestants as about the competitive answering of questions. Even a playground game of football is generally a performative and dramatic event, eliciting the gestures and celebrations of professional players seen on television, as well as the speech and exclamations of commentators, the invocation of the roar of the crowd when a goal is scored.

With video game cultures, the layers and overlappings of meta-play are particularly complex:

A group of four ten year old girls gathers around a video game console in Anna's playroom. They are playing... Pirates: The Legend of Black Kat, which features a 
female pirate [... The girls spend their time in the game searching for treasure, navigating the pirate ship from island to island and fighting off pirates and giant crabs. The game is meant for a single player, but the girls take turns with the controller... surfing the internet on Anna's laptop while waiting for their turn. They are engaged in multiple forms of play in the same space: instant messaging..., listening to music, playing games online while simultaneously watching and coaching whichever girl currently has the controller.... The game has captured their imaginations to such a degree that the play merges as they reenact the adventure pretending to be pirates engaging in play sword fights and taking turns being Katarina [the pirate protagonist] [...]

Anna: 'This went so much faster than the $1^{\text {st }}$ time but if I have to redo it again I'm gonna be so mad'.

Anna hands the controller to Molly who explores the island looking for the treasure chest eventually encountering pirates.

Molly: 'Pirates! Oh no!

I'm running through the trees...'

Molly explores the island in the game looking for clues that will lead her to the treasure.

Molly: (handing the controller to Virginia) 'Do you want to try?'

Virginia is ... typically shy and timid on the schoolyard. She reluctantly accepts the controller.

Virginia: 'You don't need to kill the monkeys 'cause they won't hurt you'

Anna: 'Press 1, 2 right when the lock comes press 1, 2 go thru the door that opened' Virginia: (Squealing) 'Crabs, crabs, crabs, eww!' (She swipes wildly at the crabs with her sword, laughing and screeching)

Dixon and Weber's analysis of this event highlights the flow between virtual and actual play with which this book started. Like Jo and Alex's designation of their father as the player of their actual Lego Racers across the floor, the girls were 'both playing at playing a videogame and playing the game', and ' $\mathrm{t}$ ]here was a multiple layering or multitasking in the casual way they incorporated the pirate game into their play, cheering on the person playing, giving suggestions, and actually enacting or improvising around it' (Dixon and Weber 2011, 487).

Bateson suggests that animal play may have been 'an important step in the evolution of communication' (Bateson 1972, 182). If my connecting this animal meta-communication to the ludic digital media of simulation seems a stretch, 
consider Bateson's own application of meta-communication to human culture: 'trompe l'oeil painting and conjurers and special effects in cinema', cultural forms commonly regarded as precursors of virtual reality technologies, or - for Jean Baudrillard - as stages in the precession of the simulacra (Baudrillard 1994). With metroptic culture,

the idea was introduced that an original achieves its significance only through copies; they have their being only in becoming. (Goldman 1998, 19)

In thinking of play as simulational - from animals to children to technoculture - it is important to note that these simulations are not deceitful: the playful 'bite' is an illusion, but dogs and monkeys know it, and the conjurer or trompe l'oeil painter aim is to impress with the technical virtuosity of their trickery, not to trick (Bateson 1972,181). As I argued earlier, simulations and virtual realities are real in their technical apparatus and their effects on our bodies. Play and playful technologies might help us rethink artificial realities of culture and communication - and the reality of artifice.

\section{Prosthetic imagination}

There are many ways that technologies and environments, whether designed for the purposes of play or not, suggest, scaffold, extend and augment imaginative play. Play in this sense is fully machinic, and imagination is extended out into, and processed by, the mechanisms of the gameworld. Imaginative play is not only the imposition or projection of a child's fantasies into the animation of dead matter or onto the environment as if it were a cinema screen. The exercise of agency in actual play is analogous to the complicated technologies of simulation and virtual worlds. Each can be understood as a set of feedback loops and the child is acted on by the machines as well as acting on them. The game event is not reducible to the actions or locations of either. The imaginative motives of play cannot be simply located in the child's mind; just as a collective game between children conjures up a real world that does not exist - not even as electrical impulses in the nervous system - so too a collective gameworld generated by a child and a machine is distributed across and between them.

To return to simulation games, ${ }^{9}$ computer simulations are artificial imaginations, modelling systems too complex for the unaugmented human mind. We delegate to them the storage and processing of dynamic non- 
linear variables, whether these be market data in the modelling of economic predictions or the ratio between the 'Outgoing' and 'Nice' aspects of a Sims character. In a simulation game, these variables play out through, and feed back to the player from, the behaviour of the virtual world and its creatures. The mechanisms of transmedia systems provide a looser system, the components of which are plugged together by individuals and groups in many different ways:

this is the question of how the imagination as produced by commercial media articulates with the imagination, agency, and creativity of diverse children going about their daily lives. (Ito 2011, 492)

Simple machines such as toys or objects co-opted as toys may serve a similar function. In the paddling pool event discussed earlier, Jo at one point picked up a plastic footpump that was lying around after having been used to inflate the pool itself. He wanted to use it to mechanize the 'tidal wave' god power previously visited on the world through the bucket. By reconnecting the pump's hose, he could get it to suck instead of blow, and he aimed to suck up water and direct it at boats and toys in a powerful jet. I wasn't happy about having a pump full of water so stopped him.

J: What are we supposed to use then?

$\mathrm{S}$ (with a touch of sarcasm): Your imagination?

J: How are we meant to use our imagination on something that's meant to be real?...

J: We're meant to try, we're trying to do it so that it's real.

In retrospect, my irritable response clearly shows I had not grasped the multifaceted workings of the boys' imaginations or the machinic environment of their gameworld. The exercise of imagination had been effected all afternoon from the spinning into being of the heterotopian and phantasmagorical world of Mr Happy in the Age of Mythology. The vivid blue water and red-striped sails looped visual and embodied memories of the computer game, whilst the other more or less randomly accumulated toys were caught up, triggering new associations and possibilities. The materiality of this environment suggested playful behaviours, and enacted them - submerging and floating, exercising cataclysmic powers on the tiny figures. Similarly, the visitation of god powers on the computer game characters is triggered by the player's decision, but the visual animation and destructive effects of the process itself is delivered by 
the machine. Divine intervention flows through the player and the machine, so it should be no surprise that in the actual game Jo was 'trying to do it so that it's real'.

\section{The protopolitics of play}

The semiotics of gesture, mimicry, and gameplaying regained their liberty in the child's activities, are disengaged from the 'trace', that is, from the dominant competence of the school teacher's language, and a microscopic event disrupts the equilibrium of local power. (Deleuze and Guattari 1983, 33)

The microscopic events of children's imaginative play including those in and around video games are phantasmagorical. In describing and opening up numerous examples of unexpected and fantastical play around media and video games from my own research and that of others, I have hoped to question the entrenched and limiting division of reality into nature and artifice, human and non-human, active and passive, offering instead an everyday ecology of material and immaterial realities, constituted by flows of information, imagination and love. On one level, I hope I have helped to counter assumptions that play with virtual media is unimaginative, commodified, mechanical, antisocial, separate from and destructive of social, imaginative, creative outdoor play. However, I do not want to perpetuate these binaries. Play with computer games may not be 'mechanical' in the popular sense of inflexible, pre-scripted and without any intelligence, but it is - like all gameworlds - machinic. Gameworlds have always been located in and formed from their environments (sand, lamp posts, fields and virtual places), have always had their technologies (knuckle bones, balls, controllers and screens) and techniques (swinging, building, twitching thumbs). For all the events of openness, negotiation and transformations of rule sets, games can also be precise systems and for all the emergent behaviours they might allow, much play with and in them is by the rules. To play Snakes and Ladders, Solitaire or Modern Warfare 2 by their rules is not to succumb to ideology but it is to render oneself passive - though partially, incompletely and interestingly. The rules of these games are a convenient artifice to facilitate and structure periods of play with others (including machines), or by oneself. Or at the very least we need to rethink the terms 'active' and 'passive'. As discussed in Chapter 5, in a cybernetic system there is a flow of agency: components act and are acted upon. 
However, this emphasis on describing and theorizing the machinic and nonhuman participants in children's video game play should not lead us to disregard concerns about human desires, anxieties, identifications and investments as players in these media technocultural events. They may not simply initiate or dominate a gameworld, but games were spun into being through the tastes, personalities, relationships and abilities - technicities - of children through and with the non-human participants (Dovey and Kennedy 2006). If code and information must be understood as real, material, of the world, then so too can the intangible yet real, embodied yet distributed, monstrous operations of human factors - perception, imagination, creativity, anxiety, play - without always already reducing these to the reassuring singularity of identity or subjectivity, and without assuming that they are unchanged in the processes. The multiple and varied transductions of the Lego video game both on- and off-screen are a reminder of the wide range of possibilities and the complexities in children's play and in the varying networks that facilitate but also shape that play.

At the molar level of the politics of children's media and toy culture, it is important that adults pay attention to and care about the actual acquisitiveness and surveillance required by online spaces for children, the reinforcement of social divisions of gender and race in the design of toys and concomitant access to digital media and technologies. Campaigns such as Let Toys Be Toys, all-girl game jams, and initiatives such as GoldieBlox open up spaces and possibilities in adult thought and children's play. ${ }^{10}$ But at the molecular level of moments and events of play these divisions are often fluid, and flow across each other and into other strange and monstrous configurations.

I would argue that it is equally as important to allow space and time for gameworlds to emerge in playgrounds, parks, streets, in and around virtual worlds. The threat to imaginative play is not ultimately video games or pink and blue toys, but decreased access to the open space of streets and parks because of traffic or fear of strangers, and the shortening of school playtimes. It is also in the well-meaning surveillance of children's play, from the attempts to ban virtual guns and knives to the channelling of play into the rhetorics and structures of learning and development. Gameworlds need actual time and space (and benign neglect) to open up their own second dimensions. Adults need to pay less attention in some ways (and the irony of this is not lost on this parent ethologist), and more in others: 
Children need their play to make the present tolerable to themselves, and to do that they need a lot of time to themselves. We should defend that need and not intrude upon it for the protection of our own past values under the guise of preparing their future. (Sutton-Smith 1994, 146)

We need a politics of opening up symbolic and material resources for boys and girls, towards a rendering of difference as malleable, insignificant, meaningless, not a policing or closing down of disapproved types of play.

Recent years (not least in our house) have seen the emergence of a household politics of time, with parental policies and negotiations, around 'screen time', injunctions and rewards regulating Internet use and gameplay. As parents our concerns over video game play have generally not been to do with digital play itself, nor the symbolic content of particular games, but the rapt attention these passional circuits engender, the hours they command sometimes at the expense of other, less intense, social and gameworlds. The temporalities of games discussed in Chapter 5 take on a different character in network culture. Organizing and conducting a collaborative World of Warcraft raid is not something that can be paused, packed away and reopened another day - it must be done now and to be interrupted is catastrophic. The timespace of the virtual and the everyday collide. This engineering of attention at the local level of particular games is inseparable from the broader temporal politics of the attention economy in which corporations fight for our media time and that of our children, and for our money through the iterative economies of micro-payments and upgrade culture, training our attention for the demands of consumption in a media world of proliferating channels and platforms (Crogan and Kinsley 2012).

As I complete this book, Jo and Alex are in their mid teens, Lego and Playmobil has been consigned to the attic, and imaginative play evident only in the occasional eruption in jokes, flights of fancy or recollections of their own play history. However, the micro-worlds of video games persist into adulthood, a bridge alongside the blurred returns and relays of TV and literature for older children and adults, Call of Duty, Mario and The Sims alongside Harry Potter and Lord of the Rings.

Their virtual media culture has changed from the stand-alone console and computer games, through their early adoption of the MMO World of Warcraft, to the interwoven playful networks of Xbox Live, Steam and social media. These platforms and the connections they form have developed as the children have grown up, but they were prefigured by (and to an extent prepared for by) the 
local hook-ups of GameBoy cables in Pokémon swaps, the asynchronous social networks of virtual worlds such as Moshi Monsters and the sharing of tiny sound files in their first mobile phones. These online gameworlds are also spaces of collusion and communion: a friend's eleven-year-old daughter has learned the 'cup game' (a very skillful, fast and rhythmic clapping game) from YouTube; the video channel has revolutionized childlore, allowing songs, dances and crazes to spread virally not via the slow and contingent routes from playground to playground (Bishop and Burn 2013).

Jo at age sixteen can still sing the theme tune to the Pokémon TV series and the two of them still return, from the varied grown-up attractions of online culture to the reassuring familiarity of a new iteration of the Pokémon DS games. Particularly in the holidays, when they spend time together again, playing Pokémon in parallel reconnects the brother-machine.

\section{Notes}

1 Letter from Virginia Lowe, Ormond, Victoria, Australia, in New Scientist, issue 2567, 2 September 2006, http://www.newscientist.com/article/mg19125671.300imaginary-friends.html. Accessed 1st April 2013.

2 See David Gauntlett's useful summary of the case against the media effects model, http://www.theory.org.uk/david/effects.htm, and Henry Jenkins' attempts to intervene in media and policy reactions to the Columbine shootings (Jenkins 1999).

3 Though video games in their interactive and reflexive play and vivid images seem to some to fatally collapse fantasy and reality, or even to actively train children to aim and shoot as a reflex (see Lister et al. 2009, 289).

4 As evidenced by home photographs that circulate through social media - for instance, toy cars were given to a young girl by parents hoping to resist gender stereotyping. They later found the cars on their backs/roofs, tucked up in dolls' beds. To cite an example from children's literature (acknowledged by Goldman as an ethnographic source in its own right - Goldman 1998, 1), My Naughty Little Sister, a story in which the toys made by the toddler's uncle are systematically transformed - a horse on wheels into a pram, a dolls' house into an oven and then (with the collusion of her friend, Bad Harry) an actual toy oven into a steam locomotive engine (Edwards 1997).

5 Rarely so directly or frankly in their popular media resources, however.

6 Interestingly, Lilli Peller suggests that the repetitious nature of play may be a process for dealing with trauma. 
'The psychoanalytic interpretation so far has stressed the emotional release gained through play. Children play in order to mitigate, to deny, or temporarily solve a conflict. In play the child recaptures for a while the omnipotence he once believed he possessed. He repeats and gradually assimilates an experience that was traumatic or a narcissistic insult. Play may help him overcome a specific fear. And, of course, play is a source of pleasure ... the playing child repeats an experience he has had, or a part of it. Repeating it, he divests it of its uniqueness. [...] Play enables the child to reexperience, to remold past impressions and events and their accompanying moods and emotions. Playful repetition provides essential, possibly indispensable steps toward concept formation' (Peller 1971 [1952], 123-124).

7 The mingling of bodies on the street, particularly after dark, is in itself a practice that makes police anxious, more so, of course, with adults than children. The effigies and festive costumes of some political demonstrations (note the recent adoption of Guy Fawkes masks in Anonymous protests) overlap with the more playful and performative carnivals of recent years. Last year, Bristol's 'zombie walk' saw a number of zombie Jimmy Saviles - a symbolic invocation as much of the carnivalesque rejection of good taste as of the recently deceased celebrity serial sex offender. Halloween costumes of 'psycho ward' mental patients on sale in British supermarkets in 2013 generated complaints from mental health charities. These inversions have political potential, but they are profoundly ambiguous.

8 This is my own observation, based on our own dog's behaviour with puppies.

9 All computer and video games are simulations, whether they represent real-world systems and environments or not. Here I am referring specifically to the genre of games, the gameplay of which is driven by the modelling of complex systems such as the domestic lives of The Sims and the urban development of SimCity (see Giddings 2014c).

10 http://www.lettoysbetoys.org.uk, http://www.bbc.co.uk/news/technology-20186937, http://www.goldieblox.com.

Sarah Grimes' Prezi keynote for Intersections/Cross-Sections (March 2013) captures many of the issues in a particularly elegant and playful way, http://prezi.com/ v73wy2bp-r-g/politics-of-play-keynote/ 


\section{Bibliography}

Aarseth, Espen. 1997. Cybertext: Perspectives on Ergodic Literature. Baltimore: Johns Hopkins University Press.

Aitken, Stuart C. and Herman, Thomas. 1997. 'Gender, power and crib geography: Transitional spaces and potential places. Gender, Place \& Culture 4(1): 63-88.

Akrich, Madeleine. 1992. 'Technology, theory and method: The de-scription of technological objects'. In Shaping Technology/Building Society: Studies in Sociotechnical Change, edited by Wiebe Bijker and John Law, 205-224. Boston, MA: MIT Press.

Alloway, Nola and Gilbert, Pam. 1998. 'Video game culture: Playing with masculinity, violence and pleasure'. In Wired Up: Young People and the Electronic Media, edited by S. Howard, 95-114. London: UCL Press.

Ang, Ien. 1985. Watching Dallas: Soap Opera and the Melodramatic Imagination. London: Methuen.

Apperley, Thomas H. and Jayemane, Darshana. 2012. 'Game studies' material turn'. Westminster Papers in Communication and Culture 9(1): 5-25. http://www. westminster.ac.uk/_data/assets/pdf_file/0009/182448/002.-Game-Studies-MaterialTurn-Thomas-H.-Apperley-and-Darshana-Jayemane.pdf. Accessed 24 September 2013.

Atkins, Barry. 2007. 'Killing time: Time past, time present and time future in Prince of Persia: Sands of time'. In Videogame, Player, Text, edited by Barry Atkins and Tanya Krzywinska, 237-253. Manchester: Manchester University Press.

— and Krzywinska, Tanya. 2007. Videogame, Player, Text. Manchester: Manchester University Press.

Avedon, Elliott M. and Sutton-Smith, Brian. 1971. The Study of Games. London: Wiley.

Baines, Ed and Blatchford, Peter. 2011. 'Children's games and playground activities in school and their role in development'. In The Oxford Handbook of the Development of Play, edited by Anthony D. Pellegrini, 260-283. Oxford: Oxford University Press.

Bakhtin, Mikhail. 1984. Rabelais and His World. Indianapolis: Indiana University Press.

Bateson, Gregory. 1972. Steps to an Ecology of Mind: A Revolutionary Approach to Man's Understanding of Himself. New York: Ballantine.

Bateson, Patrick. 2011. 'Theories of play'. In The Oxford Handbook of the Development of Play, edited by Anthony D. Pellegrini, 41-47. Oxford: Oxford University Press.

Baudrillard, Jean. 1993. Symbolic Exchange and Death. London: SAGE. 1994. Simulation and Simulacra. Michigan: University of Michigan Press. 
2011. 'The vanishing point of communication'. In The New Media e Technocultures Reader, edited by Seth Giddings, 110-117. London: Routledge.

Bazalgette, Cary and Buckingham, David (Eds). 1995. In Front of the Children: Screen Entertainment and Young Audiences. London: BFI.

Bishop, Julia and Burn, Andrew. 2013. 'Reasons for rhythm: Multimodal perspectives on musical play'. In Children, Media and Playground Cultures: Ethnographic Studies of School Playtimes, edited by Rebekah Willett, Chris Richards, Jackie Marsh, Andrew Burn and Julia C. Bishop, 89-119. New York: Palgrave Macmillan.

Bolter, Jay David and Grusin, Richard. 1999. Remediation: Understanding New Media. Cambridge, MA: MIT Press.

Briggs, Matt. 2006. 'Teletubbies, play and parenthood'. European Journal of Cultural Studies 9(4): 441-460.

Brisley, Joyce Lankester. 1973 [1928]. Milly-Molly-Mandy Stories. London: Puffin Books. Brougère, Gilles. 1999. 'Some elements relating to children's play and adult simulation/ gaming. Simulation \& Gaming 30(2): 134-146.

Bruner, Jerome S. Jolly, Alison and Silva, Kathy (Eds). 1976. Play: Its Role in Development and Evolution. Harmondsworth: Penguin.

Buckingham, David and Sefton-Green, Julian. 2004. 'Structure, agency, and pedagogy in chidren's media culture'. In Pikachu's Global Adventure: The Rise and Fall of Pokémon, edited by Joseph Tobin, 12-33. Durham, NC: Duke University Press.

Burghardt, Gordon M. 2011. 'Defining and recognizing play'. In The Oxford Handbook of the Development of Play, edited by Anthony D. Pellegrini, 9-18. Oxford: Oxford University Press.

Burn, Andrew. 2013. 'Computer games on the playground'. In Children, Media and Playground Cultures: Ethnographic Studies of School Playtimes, edited by Rebekah Willetts, Chris Richards, Jackie Marsh, Andrew Burn and Julia C. Bishop, 120-144. New York, NY: Palgrave Macmillan.

and Richards, Chris. 2013. 'Conclusion: Forms, functions and ethnographic challenge'. In Children, Media and Playground Cultures: Ethnographic Studies of School Playtimes, edited by Rebekah Willetts, Chris Richards, Jackie Marsh, Andrew Burn and Julia C. Bishop, 212-239. New York, NY: Palgrave Macmillan.

Caillois, Roger. 1962. Man, Play and Games. London: Thames \& Hudson.

Carey, James. 1992. Communication as Culture: Essays on Media and Society. London: Routledge.

Carr, Diane. 2002. 'Playing with Lara'. In ScreenPlay: Cinema/Videogames/Interfaces, edited by Tanya Krzywinska and Geoff King, 171-180. London: Wallflower.

Carrington, Victoria. 2005. 'New textual landscapes, information and early literacy'. In Popular Culture, New Media and Digital Literacy in Early Childhood, edited by Jackie Marsh, 13-27. London: Routledge.

Carroll, Lewis. 1998 [1865 and 1871]. Alice's Adventures in Wonderland and Through the Looking Glass, and What Alice Found There. London: Penguin. 
Cassel, Justine and Jenkins, Henry. 1998. From Barbie to Mortal Kombat: Gender and Computer Games. Cambridge, MA: MIT Press.

Chudacoff, Howard P. 2011. 'The history of children's play in the United States'. In The Oxford Handbook of the Development of Play, edited by Anthony D. Pellegrini, 101-109. Oxford: Oxford University Press.

Clifford, James. 1988. The Predicament of Culture: Twentieth-Century Ethnography, Literature, and Art. Cambridge, MA: Harvard University Press.

Cobb, Edith. 1977. The Ecology of Imagination in Childhood. London: Routledge \& Kegan Paul.

Cohen, David and MacKeith Stephen, A. 1991. The Development of Imagination: The Private Worlds of Childhood. London: Routledge.

Consalvo, Mia. 2007. Cheating: Gaining Advantage in Videogames. Cambridge, MA: MIT Press.

Copier, Marinka. 2005. 'Connecting worlds: Fantasy role-playing games, ritual acts, and the magic circle'. Digital Games Research Association Conference, Vancouver. http:// ir.lib.sfu.ca/handle/1892/1563. Accessed 20 September 2013.

Cordes, Colleen and MIller, Edward. 1999. 'Fool's gold: A critical look at computers in childhood'. http://drupal6.allianceforchildhood.org/fools_gold. Accessed 3 December 2013.

Crogan, Patrick and Kinsley, Samuel. 2012. 'Paying attention: Towards a critique of the attention economy’. Culture Machine 13: 1-29. http://culturemachine.net/index.php/ cm/article/view/463. Accessed 6 January 2014.

Crompton, Richmal. 1983 [1922]. Just William. London: Macmillan.

Cross, Beth. 2005. 'Mimesis and the spatial economy of children's play across digital divides: What consequences for creativity and agency?'. In Popular Culture, New Media and Digital Literacy in Early Childhood, edited by Jackie Marsh, 125-143. London: Routledge.

Cross, Gary. 1997. Kids' Stuff: Toys and the Changing World of American Childhood. Cambridge, MA: Harvard University Press.

Davis-Floyd, Robbie and Dumit, Joseph (Eds). 1998. Cyborg Babies: From Techno-Sex to Techno-Tots. Cambridge, MA: MIT Press.

De Castell, Suzanne and Jenson, Jennifer. 2004. 'Paying attention to attention: New economies for learning'. Educational Theory 54(4): 381-397.

Deleuze, Gilles. 1988. Spinoza: Practical Philosophy. San Fransisco: City Lights. 1992. 'Ethology: Spinoza and Us'. In Zone 6: Incorporations, edited by Jonathan Crary and Sandford Kwinter, 625-633. New York: Zone Books.

- 2004. The Logic of Sense. London: Continuum.

Deleuze, Gilles and Guattari, Félix. 1983. On the Line. New York: Semiotext(e). -1987. A Thousand Plateaus: Capitalism and Schizophrenia. London: Continuum. Dennett, D.C. 1971. 'Intentional systems'. The Journal of Philosophy 68(4): 87-106. 
Dixon, Shanly and Weber, Sandra. 2007. 'Playspaces, childhood, and videogames'. In Growing Up Online: Young People and Digital Technologies, edited by Sandra Weber and Shanly Dixon, 15-34. New York: Palgrave Macmillan.

2011. 'Playspaces, childhood and videogames' in The New Media o Technocultures Reader, edited by Seth Giddings, 479-490. London: Routledge.

Dodge, Martin and Kitchin, Rob. 2005. 'Code and the transduction of space'. Annals of the Association of American Geographers 95(1): 162-180.

Dourish, Paul. 2004. Where the Action Is: The Foundations of Embodied Interaction. Cambridge, MA: MIT Press.

Dovey, Jon and Kennedy, Helen W. 2006. Game Cultures: Computer Games as New Media. Milton Keynes: Open University Press.

Downey, Gary Lee, Dumit, Joseph and Williams, Sarah. 1995. 'Cyborg anthropology'. In The Cyborg Handbook, edited by Chris Hables Gray, Steven Mentor and Heidi Figueroa Sarrier. New York: Routledge, 347-362.

Edwards, Dorothy. 1997. The Complete My Naughty Little Sister Storybook. London: Egmont.

Egenfeldt Nielsen, Simon, Heide Smith, Jonas and Pajares Tosca, Susana. 2008. Understanding Video Games: The Essential Introduction. New York: Routledge.

Erikson, Erik H. 1971 [1951]. 'Sex differences in the play configurations of American pre-adolescents'. In Child's Play, edited by R.E. Herron and Brian Sutton-Smith. London: John Wiley and Sons. 126-144.

Facer, Keri, Sutherland, Ros and Furlong, Ruth. 2003. Screenplay: Children and Computing in the Home. London: Routledge.

Factor, June. 2004. 'Tree stumps, manhole covers and rubbish tins: The invisible playlines of a primary school playground'. Childhood 11(2): 142-154.

Feinburg, Sylvia G. 1976. 'Combat in child art'. In Play: Its Role in Development and Evolution, edited by Jerome S. Bruner, Alison Jolly and Kathy Silva, 589-593. Harmondsworth: Penguin.

Fiske, John and Watts, Jon. 1985. 'Video games: Inverted pleasures'. Australian Journal of Cultural Studies, 3(1). http://wwwmcc.murdoch.edu.au/ReadingRoom/serial/ AJCS/3.1/Fiske.html. Accessed 8 February 2014.

Flax, Jane. 1993. Thinking Fragments: Psychoanalysis, Feminism, and Postmodernism in the Contemporary West. Berkeley, CA: University of California Press.

Fleming, Dan. 1996. Powerplay: Toys as Popular Culture. Manchester: Manchester University Press.

Flynn, Bernadette. 2003. 'Geography of the digital hearth'. Information, Communication and Society 6(4): 551-576.

—. 2007. 'The morphology of space in virtual heritage'. In Theorizing Digital Heritage: A Critical Discourse, edited by Fiona Cameron and Sarah Kenderdine, 349-368. Cambridge, MA: MIT Press. 
Forgacs, David. 1992. 'Disney animation and the business of childhood'. Screen 53: 361-374.

Frasca, Gonzalo. 2003. 'Simulation versus narrative: Introduction to ludology'. In The Video Game Theory Reader, edited by Mark J.P. Wolf and Bernard Perron, 221-236. London: Routledge.

Fuller, Mary and Jenkins, Henry. 1995. 'Nintendo and new world travel writing: A dialogue'. In Cybersociety: Computer-Mediated Communication and Community, edited by Steven G. Jones, 57-72. Thousand Oaks: SAGE.

Fuller, Matthew. 2005. Media Ecologies: Materialist Energies in Art and Technoculture. Cambridge, MA: MIT Press.

Gauntlett, David. 1998. 'Ten things wrong with the "media effects" model'. http://www. theory.org.uk/david/effects.htm. Accessed 7 February 2014.

Geertz, Clifford. 1973. The Interpretation of Cultures: Selected Essays. New York: Basic Books.

Genosko, Gary. 2012. 'Deleuze and Guattari: Guattareuze \& Co'. In The Cambridge Companion to Deleuze, edited by Daniel W. Smith and Henry Somers-Hall, 151-169. Cambridge, MA: Cambridge University Press.

Giddings, Seth. 2003. 'Circuits: A video essay on virtual and actual play'. In Level Up: Digital Games Research Conference [CD-ROM], edited by Marinka Copier and Joost Raessens. Utrecht: Faculty of Art, Utrecht University Press.

2006. 'Walkthrough: Videogames as technocultural form' PhD thesis. University of the West of England, Bristol. http://www.sethgiddings.net/?page_id=191.

_ 2007a. " "I'm the one who makes the Lego Racers go!" Studying virtual and actual play’. In Growing Up Online: Young People and Digital Technologies, edited by Sandra Weber and Shanly Dixon, 35-49. New York: Palgrave Macmillan.

_ 2007b. 'Dionysiac machines: Videogames and the triumph of the simulacra'. Convergence: The International Journal of Research into New Media Technologies 13(3): 417-431.

-2007c. 'A 'pataphysics engine: Baudrillard, technology, play, and realities'. Games and Culture 2(4): 392-404.

2011. 'Monsters, mini-games and Mr Happy: A video essay on virtual and actual play'. Audio-Visual Thinking: A Journal of Academic Videos No. 3. http://www. audiovisualthinking.org/videos/mrhappy/. Accessed 24 January 2014.

2 2014a. 'Bright bricks, dark play: On the impossibility of studying LEGO'. In LEGO Studies: Examining the Building Blocks of a Transmedia Phenomenon, edited by Mark J.P. Wolf. New York: Routledge.

2 2014b. 'Towards the phenomenology of Angry Birds'. http://www.microethology. net/towards-the-phenomenology-of-angry-birds/. Accessed 5 February 2014.

. 2014c. 'Simulation'. In The Routledge Companion to Video Game Studies, edited by Mark J.P. Wolf and Bernard Perron, 259-266. New York: Routledge. 
Giddings, Seth and Kennedy, Helen W. 2003. 'Digital games as new media'. In Understanding Digital Games, edited by Jason Rutter and Jo Bryce, 129-147. London: SAGE.

- 2008. 'Little Jesuses and fuck-off robots: On aesthetics, cybernetics, and not being very good at Lego Star Wars'. In The Pleasures of Computer Gaming: Essays on Cultural History, Theory and Aesthetics, edited by Melanie Swalwell and Jason Wilson, 13-32. Jefferson, NC: McFarland \& Co.

- 2010. 'Incremental speed increases excitement: Bodies, space, movement and televisual change. Journal of Television and New Media 11(3): 163-179.

Giroux, Henry A. and Pollock, Grace. 2010. The Mouse that Roared: Disney and the End of Innocence (2nd Edition). Maryland: Rowman \& Littlefield.

Goldacre, Ben. 2011. 'Why won't Professor Susan Greenfield publish this theory in a scientific journal?' Bad Science. http://www.badscience.net/2011/11/why-wontprofessor-greenfield-publish-this-theory-in-a-scientific-journal/. Accessed 27 January 2014.

Goldman, L.R. 1998. Child's Play: Myth, Mimesis and Make-Believe. Oxford: Berg. Goldstein, Jeffrey H. 1994. Toys, Play, and Child Development. Cambridge, MA: Cambridge University Press.

- 2011. 'Technology and play'. In The Oxford Handbook of the Development of Play, edited by Anthony D. Pellegrini, 322-337. Oxford: Oxford University Press.

—, Buckingham, David and Brougére, Gilles. 2004. Toys, Games, and Media. Mahwah, NJ: Lawrence Erlbaum.

Göncü, Artin and Gaskins, Suzanne Baines. 2011. 'Comparing and extending Piaget's and Vygotsky's understandings of play: Symbolic play as individual, sociocultural, and educational interpretation'. In The Oxford Handbook of the Development of Play, edited by Anthony D. Pellegrini, 48-57. Oxford: Oxford University Press.

Gray, Chris Hables, Mentor, Steven and Figueroa-Sarrier, Heidi. (Eds). 1995. The Cyborg Handbook. New York: Routledge.

Green, B., Reid, J. and Bigum, C. 1998. 'Teaching the Nintendo generation? Children, computer culture and popular technologies'. In Wired Up: Young People and the Electronic Media, edited by Sue Howard, 19-42. London: UCL Press.

Haddon, Leslie. 1994. 'Explaining ICT consumption: The case of the home computer'. In Consuming Technologies: Media and Information in Domestic Spaces, edited by Roger Silverstone and Eric Hirsch, 82-96. London: Routledge.

Hall, Stuart (Ed). 1997. Representation: Cultural Representations and Signifying Practices. London: SAGE.

Hansen, Mark B.N. 2006. Bodies in Code: Interfaces with Digital Media. New York: Routledge.

Haraway, Donna. 1990. 'A manifesto for cyborgs: Science, technology, and socialist feminism in the 1980s'. In Feminism/Postmodernism, edited by Linda J. Nicholson, 190-234. London: Routledge. 
2004. The Haraway Reader. London: Routledge.

Harker, Christopher. 2005. 'Playing and affective time-spaces'. Children's Geographies 3(1): 47-62.

Hebdige, Dick. 1979. Subculture: The Meaning of Style. London: Routledge.

Herron, R.E. and Sutton-Smith, Brian. 1971. Child's Play. London: John Wiley \& Sons. Highmore, Ben. 2002. Everyday Life and Cultural Theory: An Introduction. London:

Routledge.

—. 2005. Cityscapes: Cultural Readings in the Material and Symbolic City.

Basingstoke: Palgrave Macmillan.

- 2009. A Passion for Cultural Studies. Basingstoke: Palgrave Macmillan.

Hine, Christine. 2000. Virtual Ethnography. London: Sage.

Holloway, Sarah L. and Valentine, Gill., 2000. Children's Geographies: Playing, Living,

Learning. London: Routledge.

Huizinga, Johan. 1986 [1938]. Homo Ludens: A Study of the Play Element in Culture.

Boston, MA: Beacon Press.

Hutchby, Iain and Moran-Ellis, Jo. 2001. Children, Technology and Culture: The Impacts of Technologies in Children's Everyday Lives. London: Routledge Falmer.

Hutchins, Edwin. 1995. Cognition in the Wild. Cambridge, MA: MIT Press.

Ito, Mizuko. 1998. 'Inhabiting multiple worlds: Making sense of SimCity $2000^{\text {tm }}$ in the

fifth dimension'. In Cyborg Babies: From Techno-Sex to Techno-Tots, edited by Robbie

Davis-Floyd and Joseph Dumit, 301-316. New York: Routledge.

- 2011. 'Mobilizing the imagination in everyday play: The case of Japanese media mixes. In The New Media and Technoculture Reader, edited by Seth Giddings, 491-505. London: Routledge.

Jansson, Tove. 2003 [1955]. Moominsummer Madness. London: Puffin Books. Jenkins, Henry. 1999. 'Professor Jenkins goes to Washington'. Harper's Magazine, July. http://harpers.org/archive/1999/07/professor-jenkins-goes-to-washington/. Accessed 7 February 2014.

Jenson, Jennifer and de Castell, Suzanne. 2008. 'Theorizing gender and digital gameplay: Oversights, accidents and surprises'. Eludamos: Journal for Computer Game Culture 2(1): 15-25.

Jones, Gerard. 2002. Killing Monsters: Why Children Need Fantasy, Super Heroes and Make-Believe Violence. New York: Basic Books.

Juster, Norton. 1989 [1961]. The Phantom Tollbooth. New York: Random House. Juul, Jesper. 2003. 'The game, the player, the world: Looking for a heart of gameness'. In Keynote at the Level Up: Digital Games Research Association Conference Proceedings, edited by Marinka Copier and Joost Raessens. Utrecht: Utrecht University. http:// jesperjuul.net/text/gameplayerworld. Accessed 29 January 2014.

- 2004. 'Introduction to game time'. In First Person: New Media as Story,

Performance, and Game, edited by Noah Wardrip-Fruin and Pat Harrigan, 131-142.

Cambridge, MA: MIT Press. 
Kelley, Heather. 2007. 'Animal crossing: A game in time”. In Space Time Play: Synergies between Computer Games, Architecture \& Urbanism, edited by Friedrich von Borries, Steffen P. Walz and Matthias Böttger, 180-181. Basel: Birkhauser Verlag AG.

Kelly-Byrne, Diana. 1989. A Child's Play Life: An Ethnographic Study. New York: Teachers' College Press.

Kember, Sarah. 2003. Cyberfeminism \& Artificial Life. London: Routledge.

Kinder, Marsha. 1991. Playing with Power in Movies, Television and Video Games: From Muppet Babies to Teenage Mutant Ninja Turtles. Berkeley, CA: University of California Press.

Klevjer, Rune. 2001. 'Computer game aesthetics and media studies'. Paper presented at the 15th Nordic Conference on Media and Communication Research, Reykjavik, Iceland. http://folk.uib.no/smkrk/docs/klevjerpaper_2001.htm. Accessed 4 December 2013.

—. 2006. 'Danzando con il Grottesco Moderno. Guerra, Iavoro, gioco e rituale nei First Person Shooter run-and-gun'. In Gli strumenti del videogiocare: Logiche, estetiche e (v)ideologie, edited by M. Bittanti. Milano: Costa \& Nolan. English translation. http://folk.uib.no/smkrk/docs/dancing.htm. Accessed 6 January 2014.

Kline, Stephen. 1993. Out of the Garden: Toys, TV, and Children's Culture in the Age of Marketing. London: Verso.

— Dyer-Witheford, Nick and de Peuter, Greig 2003, Digital Play: The Interaction of technology, Culture, and Marketing, Montreal: McGill-Queen's University Press.

Kücklich, Julian. 2008. 'Forbidden pleasure: Cheating in computer games'. In The Pleasures of Computer Gaming: Essays on Cultural History, Theory and Aesthetics, edited by Melanie Swalwell and Jason Wilson, 52-71. Jefferson, NC: McFarland.

Lahti, Martti. 2003. 'As we become machines: Corporealized pleasures in video games'. In The Video Game Theory Reader, edited by Mark J.P. Wolf and Bernard Perron, 157-170. New York: Routledge.

Latour, Bruno. 1987. Science in Action: How to Follow Scientists and Engineers through Society. Cambridge, MA: Harvard University Press.

— 1992. 'Where are the missing masses? The sociology of a few mundane artefacts'. In Shaping Technology/Building Society: Studies in Sociotechnical Change, edited by Wiebe Bijker and John Law, 225-258. Cambridge, MA: MIT Press.

- 1997. 'On recalling ANT'. Keynote speech, Actor Network and After workshop, Keele University. http://www.lancaster.ac.uk/sociology/research/publications/ papers/latour-recalling-ant.pdf. Accessed 28 January 2014.

Lauwaert, Maaike. 2009. The Place of Play: Toys and Digital Cultures. Amsterdam: Amsterdam University Press.

Law, John. 1992. 'Notes on the theory of the actor-network: Ordering, strategy and heterogeneity'. Centre for Science Studies, Lancaster University. http://www.comp/ lancaster.ac.uk/sociology/soc054jl.html. Accessed 15 June 2004. 
Lister, Martin, Dovey, Jon, Giddings, Seth, Grant, Iain and Kelly, Kieran. 2009.

New Media: A Critical Introduction (2nd Edition). London: Routledge.

Lowenfeld, Margaret 2008 [1935]. Play in Childhood, Brighton: Sussex Academic.

Mackay, Hugh. 1997. Consumption \& Everyday Life. London: Sage.

Mackenzie, Adrian. 2002. Transductions: Bodies and Machines at Speed. London:

Continuum.

Mackenzie, Donald and Wajcman, Judy (Eds). 1999. The Social Shaping of Technology

(2nd Edition). London: Open University Press.

Manovich, Lev. 2001. The Language of New Media. Cambridge, MA: MIT Press.

Marsh, Jackie. 2005. 'Ritual, performance and identity construction: Young people's

engagement with popular culture and media texts'. In Popular Culture, New Media

and Digital Literacy in Early Childhood, edited by Jackie Marsh, 28-50. London:

Routledge.

- (Ed). 2005. Popular Culture, New Media and Digital Literacy in Early Childhood. London: Routledge.

Marsh, Kathryn. 2008. The Musical Playground: Global Tradition and Change in Children's Songs and Games. Oxford: Oxford University Press.

Mateas, Michael. 2003. 'Expressive AI: Games and artificial intelligence'. In Level Up: Digital Games Research Conference Proceedings and CD-ROM, edited by Marinka Copier and Joost Raessens. Utrecht: Faculty of Art Utrecht University Press.

Mauss, Marcel. 2002 [1950]. The Gift: Forms and Functions of Exchange in Archaic Societies. London: Routledge.

Mäyrä, Frans. 2008. An Introduction to Game Studies: Games in Culture. London: SAGE. McLuhan, Marshall. 1964. Understanding Media: The Extensions of Man. London: Routledge \& Kegan Paul.

McNamee, Sara. 1998. 'Youth, gender and video games: Power and control in the home' In Cool Places: Geographies of Youth Cultures, edited by Tracey Skelton and Gill Valentine, 195-206. London: Routledge.

2000. 'Foucault's heterotopia and children's everyday lives'. Childhood 7(4): 479-492.

Michael, Mike. 2000. Reconnecting Culture, Technology and Nature: From Society to Heterogeneity. London: Routledge.

- 2006. Technoscience and Everyday Life. Maidenhead: Open University Press. Millar, Susanna. 1968. The Psychology of Play. London: Pelican.

Monke, Lowell 2009. 'Video games: A critical analysis'. http://www.allianceforchildhood. org/sites/allianceforchildhood.org/files/file/MONKE223.pdf. Accessed 3 December 2013.

Morley, David. 1992. Television, Audiences and Cultural Studies. London: Routledge.

Morris, Sue. 2002. 'First-person shooters: A game apparatus' In ScreenPlay: Cinema/ Videogames/Interfaces, edited by Geoff King and Tanya Krzywinska, 81-97. London: Wallflower. 
Murray, Janet. 1997. Hamlet on the Holodeck: The Future of Narrative in Cyberspace. New York: Free Press.

Newman, James. 2002. 'The myth of the ergodic videogame: some thoughts on playercharacter relationships in videogames' Game Studies 2(1). http://www.gamestudies. org/0102/newman/

- 2004. Videogames. London: Routledge. 2008. Playing with Video Games. London: Routledge.

Nitsche, Michael. 2008. Video Game Spaces: Image, Play and Structure in 3D Game Worlds. Cambridge, MA: MIT Press.

Opie, Iona. 1993. The People in the Playground. Oxford: Oxford University Press. Opie, Iona and Opie, Peter. 1969. Children's Games. Oxford: Oxford University Press.

1985. The Singing Game. Oxford: Oxford University Press.

- 1997. Children's Games with Things. Oxford: Oxford University Press.

Ormerod, Fiona and Ivanić, Roz. 2002. 'Materiality in children's meaning-making practices'. Visual Communication 1(1): 65-91.

Palmer, Sue and Dolya, Galina. 2008. 'Freedom of thought: Lev Vygotsky'. TES 11/5/2008. http://www.tes.co.uk/article.aspx?storycode=398249.

Parikka, Jussi. 2010. Insect Media: An Archaeology of Animals and Technology. Minneapolis: University of Minnesota Press.

Parten, Mildred B. 1971 [1933]. 'Social play among preschool children'. In Child's Play, edited by R.E. Herron and Brian Sutton-Smith, 83-95. London: John Wiley \& Sons.

Pearson, Keith Ansell. 2002. Philosophy and the Adventure of the Virtual: Bergson and the Time of Life. London: Routledge.

Pellegrini, Anthony D. 2011. The Oxford Handbook of the Development of Play. Oxford: Oxford University Press.

Peller, Lili E. 1971 [1955]. 'Models of children's play'. In Child's Play, edited by R.E. Herron and Brian Sutton-Smith, 110-125. New York: John Wiley.

Plowman, Lydia. 2007. “"Hey, Hey, Hey! It's time to play”: Children's interactions with smart toys' In Toys, Games, and Media, edited by Jeffrey Goldstein, David Buckinghamand Gilles Brougère, 207-233. Mahwah NJ: Lawrence Erlbaum.

Pullman, Philip. 1995. Northern Lights. London: Scholastic.

Ransome, Arthur. 2012 [1930]. Swallows and Amazons. London: Vintage.

Rehak, Bob. 2003. 'Playing at being: Psychoanalysis and the avatar'. In The Video Game Theory Reader, edited by Mark J.P. Wolf and Bernard Perron, 103-128. New York: Routledge.

Richards, Chris. 2013. 'Framing and interpreting children's play'. In Children, Media \& Playground Cultures, edited by Rebekah Willett, Chris Richards, Jackie Marsh, Andrew Burn and Julia C. Bishop, 68-88. New York: Palgrave Macmillan.

Rosen, Michael. 1993. We're Going on a Bear Hunt. London: Walker Books.

Rowling, J.K. 1997. Harry Potter and the Philosopher's Stone. London: Bloomsbury. 
Salen, Katie and Zimmerman, Eric. 2003. The Rules of Play: Game Design Fundamentals. Cambridge, MA: MIT Press.

Scarlett, George W., Naudeau, Sophie, Salonius-Pasternak, Dorothy and Ponte, Iris. 2005. Children's Play. London: SAGE.

Schechner, Richard. 1998. 'Playing. Play \& Culture 1: 3-19.

Sefton-Green, Julian. 2004. 'Initiation rites: A small boy in a Poke-world'. In Pikachu's Global Adventure: The Rise and Fall of Pokémon, edited by Joseph Tobin, 141-164.

Durham, NC: Duke University Press.

Shields, Rob. 2006. 'Virtualities'. Theory, Culture \& Society 23(2-3): 85.

Silverstone, Roger. 1999. Why Study the Media? London: Sage.

Simon, Bart 2007 'Human, all too non-human: coop AI and the conversation of action' http://www.digra.org/digital-library/publications/human-all-too-non-human-coopai-and-the-conversation-of-action/

Simon, Egenfeldt Nielsen, Jonas, Heide Smith and Susana, Pajares Tosca. 2008. Understanding Video Games: The Essential Introduction. New York: Routledge.

Smith, Cynthia R. 2005. 'The CD-ROM game: A toddler engaged in computerbased dramatic play'. In Popular Culture, New Media and Digital Literacy in Early Childhood, edited by Jackie Marsh, 108-125. London: Routledge.

Smith, Peter K. 1994. 'The war play debate'. In Toys, Play, and Child Development, edited by Jeffrey H. Goldstein, 67-84. Cambridge, MA: Cambridge University Press.

Stallabrass, Julian. 1993. 'Just gaming: allegory and economy in computer games' New Left Review 198, March-April. http://newleftreview.org/I/198/julianstallabrass-just-gaming-allegory-and-economy-in-computer-games

Stevens, Reed, Satwicz, Tom and McCarthy, Laura. 2008. 'In-game, in-room, in-world: Reconnecting video game play to the rest of kids' lives'. In The Ecology of Games: Connecting Youth, Games, and Learning, edited by Katie Salen, 41-66. Cambridge, MA: MIT Press.

Stewart, Susan. 1989. Nonsense: Aspects of Intertextuality in Folklore and Literature. Baltimore: Johns Hopkins University Press.

Stone, Gregory. 1971. 'The play of little children'. In Child's Play, edited by R.E. Herron and Brian Sutton-Smith, 4-14. London: Wiley.

Suchman, Lucy. 2007. Human-Machine Reconfigurations: Plans and Situated Actions. Cambridge, MA: Cambridge University Press.

Sudnow, David. 2011 [1979]. 'Eyeball', from Pilgrim in the Microworld: Eye, mind and the essence of video skill'. In The New Media and Technocultures Reader, edited by Seth Giddings, 321-325. London: Routledge.

Sutton-Smith, Brian. 1971. 'Boundaries'. In Child's Play, edited by R.E. Herron and Brian Sutton-Smith, 103-106. New York: John Wiley.

- 1986. Toys as Culture. New York: Gardner Press.

1994. 'Does play prepare the future?'. In Toys, Play, and Child Development, edited by Jeffrey Goldstein, 130-146. Cambridge, MA: Cambridge University Press. 
- 1997. The Ambiguity of Play. Cambridge, MA: Harvard University Press.

Swift, Jonathan. 1992 [1726]. Gulliver's Travels. Ware: Wordsworth Classics.

Taffel, Sy. 2013. 'Scalar entanglement in digital media ecologies'. NECSUS European Journal of Media Studies 3. http://www.necsus-ejms.org/scalar-entanglement-indigital-media-ecologies/. Accessed 23 January 2014.

Taylor, T.L. 2006. Play Between Worlds: Exploring Online Game Culture. Cambridge, MA: MIT Press.

-2009. 'The assemblage of play'. Games and Culture 4(4): 331-339.

The Brontës. 2010. Tales of Glass Town, Angria, and Gondal: Selected Early Writings. Oxford: Oxford University Press.

Thornham, Helen. 2011. Ethnographies of the Videogame: Gender, Narrative and Praxis. Farnham: Ashgate.

Tobin, Joseph (Ed). 2004. Pikachu's Global Adventure: The Rise and Fall of Pokémon. Durham, NC: Duke University Press.

Tolkein, J.R.R. 1991 [1937]. The Hobbit, or There and Back again. London: Harper Collins.

Turkle, Sherry. 1984. The Second Self: Computers and the Human Spirit. London: Granada.

1998. 'Cyborg babies and cy-dough-plasm: Ideas about self and life in the culture of simulation'. In Cyborg Babies: From Techno-Sex to Techno-Tots, edited by Robbie Davis-Floyd and Joseph Dumit, 317-329. New York: Routledge.

_ 2005. 'Computer games as evocative objects: From projective screens to relational artifacts'. In Handbook of Computer Game Studies, edited by Joost Raessens and Jeffrey Goldstein, 267-282. Cambridge, MA: MIT Press.

Turner, Victor. 1982. From Ritual to Theatre: The Human Seriousness of Play, New York: PAJ Publications.

Urwin, Cathy. 1995. 'Turtle power: Illusion and imagination in children's play’. In In Front of the Children: Screen Entertainment and Young Audiences, edited by Cary Bazalgette and David Buckingham. 127-140. London: BFI.

Walkerdine, Valerie. 2007. Children, Gender, Video Games: Towards a Relational Approach to Multimedia. Basingstoke: Palgrave Macmillan.

Walther, Bo Kampfmann. 2003. 'Playing and gaming: Reflections and classifications' Game Studies 3(1). http://www.gamestudies.org/0301/walther. Accessed 29 January 2013.

Wasko, Janet. 2010. 'Children's virtual worlds: The latest commercialization of children's culture. In Childhood and Consumer Culture, edited by Vebjørg Tingstad and David Buckingham, 113-129. New York: Palgrave Macmillan.

Weber, Sandra and Dixon, Shanly (Eds). 2007. Growing Up Online: Young People and Digital Technologies. New York: Palgrave Macmillan.

Wegener-Spöhring, Gisela. 1994. 'War toys and aggressive play scenes'. In Toys, Play, and Child Development, edited by Jeffrey H. Goldstein, 85-109. Cambridge, MA: Cambridge University Press. 
Weinbren, Grahame. 2002. 'Mastery (Sonic, C’est Moi)'. In New Screen Media: Cinema/ Art/Narrative, edited by Martin Reiser and Andrea Zapp, 179-191. London: BFI.

Wiener, Norbert 1961. Cybernetics: Or, Control and Communication in the Animal and the Machine. Cambridge MA: MIT Press.

- 1988 [1950]. The Human Use of Human Beings: Cybernetics and Society. Boston, MA: Da Capo.

Willett, Rebekah. 2011. 'An ethnographic study of preteen girls' play with popular music on a school playground in the UK'. Journal of Children \& Media 5(4): 341-357.

—, Richards, Chris, Marsh, Jackie, Burn, Andrew and Bishop, Julia C. (Eds). 2013.

Children, Media and Playground Cultures: Ethnographic Studies of School Playtimes. New York: Palgrave Macmillan.

Williams, Raymond. 1974. Television: Technology and Cultural Form. London: Fontana. Willis, Paul. 1978. Profane Culture. London: Routledge and Kegan Paul.

Winnicott, D.W. 1974. Playing and Reality. Harmondsworth: Penguin.

Wise, J. Macgregor 2011 'Intelligent agents'. In The New Media \& Technocultures Reader, edited by Seth Giddings. 188-200. London: Routledge.

Wolf, Mark J.P. and Perron, Bernard (Eds). 2003. The Video Game Theory Reader. New York: Routledge.

—. and Perron, Bernard (Eds). 2009. The Video Game Theory Reader 2. New York: Routledge.

Woolgar, Steve. 1991. A Sociology of Monsters: Essays on Power and Technology and Domination, edited by John Law, 58-99. London: Routledge.

Woolley, Benjamin. 1992. Virtual Worlds: A Journey in Hype and Hyperreality. Oxford: Blackwell. 


\section{Index}

101 Dalmatians 71

Aarseth, Espen 86, 94, 111

activity see agency; passivity

actor-network theory 57,58

addiction 9

adolescence $53 \mathrm{n}$

Advance Wars 103, 106

adventure games $9,86,107,111$

adventure play grounds $117-35$

advergaming 73

advertising $38,70,73$

aesthesis 87

aesthetics 48, 64-6, 87, 90, 94-6,112, $115 \mathrm{n}, 149$

aesthetics of control 90, 112

affect $27,28,47,48,49,62,63,65,105$, $108-9,134,137$

Age of Mythology 22-6, 97, 111, 146-7, 156

agency $27-8,31,53,55,56,58,61,62,90$, $101,139,157$

see also passivity

agents $19,31,55,58,65,79,85,89,91-2$, $100,102,103,111-12,114$

Agon see competition

AI see artificial intelligence

Aitken, Stuart C. 124

Akrich, Madeleine 51, 58

algorithms 9, 14, 15, 91, 93, 97, 99, 104,

111,150

see also code; software

Alice Through the Looking Glass 11

Alloway, Nola 39

amplification 112

Anakin Skywalker 119, 122

Ang, Ien 55

Angria 6

animals $8,41,42-3,45,62,123,144$, $151-3,155,161 \mathrm{n}$ see also virtual animals

Animal Crossing 67, 100, 103, 105-9, 111
Animal Crossing: New Leaf 107-9

Animal Crossing: Let's Go to the City 105-9 animation/cartoons 19, 27, 28, 30, 40, 52, $63,65,67,70,76,112,113,115 \mathrm{n}$, $134,140,147-8,156$

ANT see actor-network theory

anthropology 8, 10, 50, 61, 62-3, 108, 109, 145-6

of technology 51, 57-60

anthropomorphism 102

arcade games 92

artificial intelligence 9, 65, 90, 91, 100-5, 111-12

artificial life 14

artifice/artificial $4,5,7,10,14,42,53,60$, $62,65,79,90-3,99,100-2,107$, $123,125,139,155,157$

as-if play 7, 14, 100, 104, 126, 134, 137-9

Assassin's Creed 21, 26

assemblages $28,60-1,66,113,128,139$

attention, attention economy $47,74,88$, 159

audiences 55

autism 16n

automata, software $65,92,96,100-5$ see also NPCs

Autopoesis see Poesis

Avatars 21, 28-30, 32, 94-7, 104, 114, $115 \mathrm{n}, 125,126$

Avedon, Elliott M. 43, 60

babies $37,42,53 \mathrm{n}$

badminton 44

bad play $139,140-3$

Baines, Ed 44

Baker, Shirley 2

Bamzooki 11-14

Barbie 72

Bataille, Georges 56

Bateson, Gregory 62-3, 109, 114, 123, 134, $144,150,151-3$

Baudrillard, Jean 108, 155 
Bazalgette, Cary 86

BBC 12, 14, 115n

Bear Hunt 6

Bearland 7

becoming 40, 48, 113

bedtime stories 82,88

behaviour 12-13, 14, 39, 43, 62, 65-6, 91, 93-4, 99, 104, 113, 137, 145

Beyoncé 49

biology 123

Bishop, Julia 160

Blatchford 44

Blizzard 115n

Blyton, Enid 6

BMX 129

board games $26,67,70,76,87,89$

bodies/embodiment $3,8,13,15,27,28$,

$32,41,49,56,58,61-2,65,71,77$,

$94,112,114,118-20,122,125$, 128-32, 137, 148

Bolter, Jay David 46

books $6-7,9,10,15,26,67,70,79,87,88$, 89,92

bots see robots

broadcasting 14

Briggs, Matt 64

British Library $135 n$

Brontë, Anne 6

Brontë, Charlotte 5, 6

Brooks, Mel 122

Brougère, Gilles 71

Brownie the pretend mouse 137

Bruegel, Pieter the Elder 38

Bruner, Jerome S. 43

Buckingham, David 86

Bulbasaur 85

Bullet Bill 110-11

bullying 42

Burghardt, Gordon 41

Burn, Andrew 21, 32, 117, 125-6, 140, 160

Buzz Lightyear 144

Caillois, Roger 3, 5, 13, 32, 41-2, 56, 88n, $126,130,137,148$

Call of Duty 102, 159

Call of Duty 2, 104-5

Call of Duty: Modern Warfare 2, 21, 143, 157

capitalism 38, 73-6, 108, 138 card games $9,43,44,67,70,75,79,85,87$, 89,92

Carey, James 50

carnival, the carnivalesque $37,50,126$, $132-5,139,147-8,150,161 \mathrm{n}$

Carr, Diane 94-5

Carrington, Victoria 67

Carroll, Lewis 5

cartoons see animation; television

Cassell, Justine 39

catoptric culture 146

CBBC see BBC

cell phones see mobile phones

centrifugality 9,130

centripetality 9, 129

Championship Manager 97, 115n

Chaplin, Charlie 78

chasing 21, 44, 130

see also kiss-chase

chatbots 100, 105

cheats (in computer games) $75,99,115 n$

cheating (in actual games) 120

chess $87,101-2,103$

childhood 10, 37-41, 53n, 67, 68-76

children's literature 10

see also books

Chudacoff, Howard P. 69-70, 90

cinema see films

clapping games 77,160

class $38,48,63,138$

Clifford, James 10, 56

climbing frames 118, 123

Club Penguin 73

Cobb, Edith 63, 123, 125

co-creation see participatory culture

code $9,27,65,66,93,113,115 \mathrm{n}, 137,158$

see also algorithms; software

Cohen, David 6-8, 150

Coleridge, Samuel Taylor 146

collusion $69,79,108,146,160$

comedy 87,131

comics $2,4,9,19,28,68,74,81,84,86$

communication $14,46,48-50,51,60,63$, $105,111,144,146,150$

communion see ritual

competition, competitive play $2,8,12,17$, $18,19,41-4,63,68,92,105,119$, $127,135 n$ 
computer-controlled characters see NPC computer-mediated communication 109 configuration $28,47,75,87,94,95$, 112-14, 158

Consalvo, Mia 115n

construction toys 90 see also Lego

consumer, child as see consumption consumption $8,38,55,65,67,69,70$, $73-4,78,86,159$

control aesthetics see aesthetics of control convergence 73,75

see also transmedia systems

cops and robbers 44, 126

counting-out 130

Crogan, Patrick vi, 159

Cross, Beth 146

cultural studies $51,55,61$

cup game 160

cyberculture $57,109-12$

cybernetics $14,27,33 \mathrm{n}, 58,59,62-3,86$, 92, 94, 99, 109-14, 142, 143, 157

cyberpunk 109, 114

cyberspace $47,55,76$

cybertext 86

cyborg anthropology see anthropology of technology

cyborgs $41,59,94,114$

dancing, dancing games $36,49,52,77-9$, $88,153,160$

Darth Maul 130-1, 132-5

Darth Vader 122

Davis-Floyd, Robbie 41

daydreams 5, 153

'death' in games 28-30, 113

deep description 64

Defender 111

Deleuze, Gilles 40, 47, 57, 62, 134, 157

dementia $16 n$

Dennett, Daniel 101-2, 103

desires 40, 56, 70, 158

development, rhetoric of $38,39,57$, 144

Disney 49, 70-71, 72-3, 76

distributed cognition 114

distributed imagination 92, 112-14, 118, 125-7
Dixon, Shanly vi, 15, 20-21, 51, 72-3, 88, 154

Dodge, Martin 27

dolls $6,57,76,90,144,160$ n

dolls houses 26, 90, 126

Donkey Kong 96

Dourish, Paul 66n

Dovey, Jon vi, 50, 158

Downey, Gary Lee 58

drawing $8,18,40,65,77,79-85,86,88$

dreams, play as dream-like $16 \mathrm{n}, 56,127-8$, $145,149,150$

driving games see racing games; Lego Racers 2

Dumit, Joseph 41

DVDs 40

ecology, media ecology 60, 62-3, 65, 67, $73,80,89,104,114,118,123-4,157$

economics (of children's culture) 50, 70

ectoplasm 80

education see learning; school

Edwards, Dorothy 160n

Elastigirl 145

email 47, 109

embodiment see bodies

emergence 47, 61, 65, 97

Empire Strikes Back, The 121

entertainment supersystems see transmedia systems

entropy $115 n$

Erikson, Erik H. 149

ethnicity 76, 138, 158

ethnography $10,20,42,55-7,61,64,69$, $74,75,104,123,143,145-6,153$, $160 \mathrm{n}$

ethology 10, 60, 61-3, 66, 69, 90, 118, 158 see also microethology

ethos 60, 62-3

see also ethology

events $35,52,55,58,62,63,114,128,155$

everyday life $3,4,15,35,38,41,44,51-3$, $56,75,137,139,149$

Facebook 73

Facer, Keri 51

FaceTime 59

Factor, June 124 
fairy tales $21,77,115$ n

families $1,18,35,39,63,76,97,127,135 \mathrm{n}$, $138,144-5$

see also parents

fantasy $9,10,15,44,69,71,86,137,139$, 140,155

Faraway Tree, The 6

feedback 15, 31, 32, 52, 109-12, 124, 155

Feinburg, Sylvia 80, 84

festivals see carnival

FIFA 13, 106, 143

films $4,5,6,9,19,21,26,46,48,64,67$,

$68,70,71-3,74,76,77,86,87-9$,

$92,95,96,117,119-20,122,131$,

$134,140,144-5,147,153,155$

film studies 95

first person shooters $9,68,92,102,104-5$

Fiske, John 16n

Fleming, Dan 35

flying 78, 124, 126

Flynn, Bernadette 51

Folklore, childhood 2, 21, 77, 148, 160

football 43, 97, 148, 153

'free play' see imagination/imaginative play; fantasy

Freud, Sigmund 149

friendship 79

Froebel, Friedrich 123

Frozone 145

Fuller, Matthew 60

Fuller, Mary 96

Furby 91, 104

games see adventure games; board games; card games; platform games; playground games; sandbox games; simulation games; tabletop games

game jams 158

game shows 89

game studies 8, 43, 95

Gauntlett, David 160n

Geertz, Clifford 56

gender 38-9, 48, 49, 63, 76, 138-9, 140, 158

gestures $39,63,71,78,84,117,143$

ghosts $21,22,103,120,122,147-8,151$

Gibson, William 47, 109

Giddings, Seth 23, 56, 64, 66n, 93, 96-7, $115 \mathrm{n}, 145,161 \mathrm{n}$ gift economies 56, 108-9

Gilbert, Pam 39

Giroux, Henry 70-1

Glass Town 6

god powers 25

Gogo's Crazy Bones 22-3, 33n

Goldacre, Ben 16n

GoldieBlox 158

Goldman, L.R. 145-6, 155, 160n

Goldstein, Jeffrey H. 91

Gondal 6

gorillas 40

Grable, Betty 77-8

Grand Theft Auto 30, 67, 87, 126-7, 150

Grant, Iain Hamilton vi, 62

gravity $44,126-7,129-30$

see also virtual physics

Gray, Chris Hables 58

Greenfield, Susan 16n

Grimes, Sarah 161n

Groteque, the 147-9

Grusin, Richard 46

Guattari, Félix 40, 57, 134, 157

Guild Wars 2, 111

Gulliver's Travels 5

gurning 36

Habbo Hotel 73

Haddon, Leslie 115n

Hall, Stuart 49

Halloween 131, 150, 161n

hammocks 128

Hamville 7

Hansen, Mark 66n

Haraway, Donna 10, 57, 59

Harry Potter 9, 19, 92, 159

HCI 66n

Hebdige, Dick 55

Herman, Thomas 124

Herron, R.E. 39, 43, 53n, 133

hide-and-seek 60

Highmore, Ben 51, 56, 63

High School Musical 78

Hine, Christine 56

His Dark Materials 6

Holloway, Sarah L. 51

hopscotch 50, 123

Hoth 120-1, 130

Huizinga, Johan 3, 8, 56, 151-2 
Huli people 145-6

human see non-humans

humanism 57, 58

humanities 57, 58, 62

Hurlock, Elizabeth B. 53n

Hutchby, Iain 37, 51

Hutchins, Edwin 114

hyperreality 139

hypersociality 75-6

identity, identification $32,55,67,76-9$, $95-9,113,131,133,138,158$

ideology 48, 68, 76-7, 139, 157

imaginary friends 137

imagination/imaginative play $38,39,40$, $56,63,66,69,70,71,72-3,74,76$, $86,99,113-14,117-35,137-61$

see also distributed imagination

imagineering 70, 138

immateriality $13,15,46,47,62,65,88$, $114,117-35,137,157$

see also materiality

immersion 15, 46, 69

Incredibles, The 124, 145

infants see babies

information $28,52,63,67,125,157,158$

intentional stance 101-2, 104

interactivity/interactive media $86,90-1$, 94,111

interfaces 50, 93, 94, 97

interkingdoms 40

Internet $15,47,67,73,86,89,138,154$, 159

interpassivity $31-2$

intersubjectivity 118

iPad 59

see also tablet computers

Ito, Mizuko 75-6, 85-6, 156

Ivysaur 84-5

Jansson, Tove 5

Jenkins, Henry 39, 96, 160n

JLS 78

jokes $39,42,77,86,89,147-8,159$

jumping 84, 95, 126

Just William 6

Juster, Norton 5

Juul, Jesper 43, 106

Juville 7 kaleidoscopes 90

Kelley, Heather 108

Kennedy, Helen W. vi, 50, 66n, 93, 96-7, 115n, 158

kinaesthetics $2,64-6,77,96,121,128-9$, 131,134

kindergarten see pre-school

King of the Castle 123

Kinsley, Sam 159

kiss-chase 78

Kitchin, Rob 27

Klevjer, Rune vi, 90, 148

Kline, Stephen 70

knuckle bones 33n, 157

krakens 23-6, 146-7

Kücklich, Julian 115n

labyrinths see mazes

language 16n, 42

Lara Croft 94-5, 96

Latour, Bruno 10, 27, 57, 58, 59, 102

laws see rules

learning $38,42-3,69,76,90,144$, 158

Legend of Zelda 111

Legend of Zelda: Phantom Hourglass, The 106

Lego 6, 17-9, 25, 26, 27, 28-32, 46, 67, 96-7, 112-14, 115n, 125, 140-2, $144,149,159$

Lego Racers 2, 18-19, 26-32, 35, 46, 61, 63, $64,112-14,142,158$

Lego Star Wars 89, 96-7, 115n, 117, 121

Let Toys Be Toys 158

Librande, Stone $115 \mathrm{n}$

light sabres 117, 121-2, 131, 134

liminal, the 50

liminoid, the 50,133, 150

Lister, Martin vi, 46, 47, 73, 87, 94, 101, $115,160 \mathrm{n}$

Little Big Planet 100

Little Wars 6

Longo, Robert 126

Looney Tunes 110

Lord of the Rings 92, 159

love 157

Lowenfeld, Margaret 99, 123

ludus $88 \mathrm{n}$

Luke Skywalker 119, 120 
machines 41, 58, 62, 63, 94, 111, 137, 157

machinic, the $10,76,88,104,112,156$, 157-8

Mackay, Hugh 51

MacKeith, Stephen 6-8, 150

Mackenzie, Adrian 27

Mackenzie, Donald 51, 58

magic 71, 115n, 140, 144, 148

make-believe see as-if play

Manovich, Lev 97

marbles 44, 123

Mario 95-6, 159

Mario Kart 110-11

Marsh, Jackie 49-50, 77

marvellous, the 56, 114, 144

massively multiplayer online games 91,92

Mateas, Michael 100, 103

materiality $55,59,77,88,117-35,156$

see also immateriality

material-semiotic 27

Mauss, Marcel 56, 108, 109

Maxis 14

mazes 22, 50, 112

McCarthy, Laura 97-9

McLuhan, Marshall 48, 49, 60, 68

Mead, Margaret 123

meaning $10,48-9,76,87$

media ecology see ecology

media effects 140

media studies $48,51,55,61,63,87,94,95$

mediation 49

Mega Bloks 17-18

merchandizing $70,73,74$

meta-communication 151-5

see also meta-play

meta-games see meta-play

Metal Gear Solid 96

meta-play 5, 69, 80, 85, 113, 127, 139, $150-5$

metroptic culture 146, 155

Michael, Mike 51

Mickey Mouse Club 70

microethnography 61

see also microethology

microethology vi, 55-66, 114, 118

Microsoft Excel 115n

microworlds $13,30,50,61$

Midsummer Night's Dream, A 15
Milly-Molly-Mandy 6

mimesis $48,68,71,76,117,138,144-6$

mimicry 5,126

Minecraft 98, 100

Minesweeper 100

mini-games 23, 113, 126, 128, 131, 134, 142

mobile games 92

mobile phones $51,89,90,114,115 \mathrm{n}, 143$, 160

mobile privatization 38

Monopoly 43

Monroe, Marilyn 78

monsters 14, 36-7, 121, 147-8, 158

Moran-Ellis, Jo 37, 51

Morley, David 55

Moshi Monsters 91, 160

multi-user domains (MUDs) 109

Murray, Janet 90

music 49,77

music hall songs 77

musicology 77

My Little Pet Shop 78

My Naughty Little Sister 160n

narrative $4,5,9,19,26,64,68,69,71,76$, $86,88,92,112,117,138$

nature $1-3,10,57,58,62,69,118,122-3$, 125,157

Neopets 73-4

Neopia 74

Newman, James 94, 96

Nintendo 96, 105

Nintendo 3DS 107

Nintendo DS 1, 85, 106, 160

Nintendo GameBoy 74, 75, 85, 160

Nintendogs 91

Nintendo Wii 106

Nitsche, Michael 93

non-humans $40,44,52,55,56,57,58,59$, $61,91-92,99,101-2,104-6,112$, $139,157,158$

non-player characters see NPCs

nonsense $22,45,49,51,131,139,147-50$

norse myths 23

NPCs 18, 44, 90, 91, 100-5, 107-9, $110-11,112$

see also automata, software

nursery rhymes 77 
Obi Wan Kenobi 122

Oculus Rift 46

Opie, Iona $39,40,68,77,78,123-4,130$, $142,148-9$

Opie, Peter 77, 78

oral culture 69 see also Folklore, children's

outdoor play $15,68,69,117-35$, 157

see also playground games

Pac-Man 103

paidia $32,88 n$

Papua New Guinea 145-6

paracosms $6-8,10,20,135,150$

parents $9,18,31,60,64,76,79,88,128$,

$142,144,158,159,160 \mathrm{n}$

see also families

Parikka, Jussi 60, 65

parody 131,138

participatory culture 73-6

passivity $8,31-2,53,85-7,93-4,112,113$, 139,157

PayPal 74

peek-a-boo 44

Pellegrini, Anthony D. 43

Peller, Lili 127-8, 160n

Penny Dreadfuls 68

performance $26,31,39,49-50,51-2,55$,

$71,76,78,85,86,87,117,120,132$, $133,146,152$

Petsland 6

Petsville 7

Phantasmagoria (play as) $26,57,66,118$, $133,139,146-50,156$

Phantom Tollbooth, The 5

phenomenology 66n

Photo Booth 36, 45, 52

Photorealism 10

Pichu 83

Pidgey 84

Pikachu 83, 84

Pikmin 20-1, 26

pinball 9

pirates $22-6,128,131,134,153-4$

Pirates: The Legend of Black Kat 154-5

plateaus 134

platform games 9,92 playground games $9,14,15,21-2,39,40$, $49,67,68,69,71,77-9,89,117-35$, $138,140,142,147-9,151,158$

playing cards see card games

Playmobil 23, 159

PlayStation 3, 59

playtime, school $3,8,21,38,72,78,127$, 158

Plowman, Lydia 76

Poesis 6, 48, 145-6

poetry $122-3$

Pokémon 1, 2-3, 6, 13, 14, 33n, 67, 73, $74-5,77,79-85,91,160$

Pollock, Grace 70-1

Possumbul World 7

post-digital, the 118

posthuman, the 41,59

potlatch 108

Power Rangers 71

power-ups 96, 110-11

pre-school 41, 57, 90

prosthetic imagination 127

Primape 84-5

psychoanalysis $149,160-1 \mathrm{n}$

psychology 8, 27, 42, 44, 60, 109, 114, 115n, 127, 144, 149-50

Pullman, Phillip 6

puzzles $2,9,75,86,92,95,97,112$

quests $89,120,127$

quizzes 68,153

race see ethnicity

racing games $110-11$

Raichu 83

reality 47,50

real-time strategy games 103, 106

reality television 89

repetition in play $40,49,71,87,91,118$, $127-8,142,147,160-1 \mathrm{n}$

representation see mimesis

Return of the Jedi, The 120

Richards, Chris 124, 140, 143, 145

ritual 14, 43, 49-50, 56, 79, 152, 160

Robin Hood: Men in Tights 122, 131

robots $90,91,115 \mathrm{n}, 142,146,148,149$, 151

Rogers, Ginger 78 
role play $32,44,77,132-3,144$

role-playing games see RPGs

Rosen, Michael 6

rough and tumble play $44,65,72,84-5$, 142

Rowling, J.K. 5

RPGs 111

Rugrats 67

rules $9,14,21,41,44,92-4,117,120,122$, 126,138

Salen, Katie 109-10, 152

Salome 78

sandbox games 9

sand play 99, 123

Satwicz, Tom 97-9

Scarlett, George W. 142

Schechner, Richard 41

schismogenesis 62

school 38, 39, 123, 145, 157

see also pre-school

Science \& Technology Studies 57

see also sociology of technology; anthropology of technology

science fiction 109

screen time 159

Sefton-Green, Julian 64, 74-5

sexuality, children's $49,78,148-9$

Shakespeare, William 3-4

Shields, Rob 47-8

Silverstone, Roger 4, 16n, 49S

SimCity 97, 98, 107, 115n, 161n

Simon, Bart 104-5

Simpsons, The 131

Sims, The 13-14, 91, 93, 97, 100, 107, $126-7,145,156,159,161 \mathrm{n}$

Sims Pets 91

simulation $5,13,26,27,28,46,47,90,92$, $108,109,115 \mathrm{n}, 145-6,152,154-5$, $161 \mathrm{n}$

simulation games 9, 92, 97-9, 145, 155-6, $161 \mathrm{n}$

see also Sims, The and SimCity

singing games/songs $36,39,40,49,63$, $77-9,80,147,150,152,160$

Situationist International 56

skateboarding 129-30

skipping 44, 65, 78-9, 123, 124
Skype 59

smart phones see mobile phones

smart toys 90-1, 100, 126

Smith, Peter K. 140, 142

SMS 42

Snakes and Ladders 87, 92, 157

Snow White 71-2, 77, 88, 92

socialism, municipal 108

social media 47, 51, 69, 159

social sciences $57,58,62$

Social Shaping of Technology (SST) 58

sociology of technology 51

software 2, 9, 12-13, 19, 23, 29, 31, 32, $36,45,47,51,52,55-6,61,63$, 64-6, 77, 88, 89-115, 125-7, 137

see also algorithms; code

Solid Snake 96

solitaire 44,157

songs see singing games/songs

Sonic the Hedgehog 96, 106-7

sort-of see as-if

Space Invaders 92

spectators 5

Spinoza, Baruch 62

sport 2, 3, 5, 8, 42, 68, 76, 98, 127

Spy Kids 145

Stallabrass, Julian 94

Star Wars 117-22, 128-35, 149

see also Lego Star Wars and Star Wars Battlefront II

Star Wars Battlefront II 132

Starjumper 120

stealth mode 21

Steam 159

Stevens, Reed 27, 97-9

Stewart, Susan 149-50

Stone, Gregory 37-8

STS see Science \& Technology Studies

subjectivity 62

see also identity

Suchman, Lucy 66n

Sudnow, David 13

superheroes 124, 135

Super Mario Bros. 96

Super Mario Galaxy 96

Super Monkey Ball 100

surrealism 44, 56-7 
Sutton-Smith, Brian 8, 39, 43, 48, 53n, 60, $71,133,139,142,144,145,147$, 150, 159

Swallows and Amazons 6

Swift, Jonathan 5

sword-fighting 121, 131, 134

symbolic exchange 108-9

tabletop games 9

tablet computers 41,90 see also iPad

Tae Kwon Do 135n

Taffel, Sy 60

Tajiri, Satoshi 6

Tamagotchi 68, 91

Taylor, T.L. $60-1$

technicity 158

technoculture 15, 145, 155

technological imaginary 16n, 109

television $2,3,4,6,12,19,27,38,48,55$, $67-8,69,70,71,74,86,88,89,101$, $119,126,140,147,153$

Temple, Shirley 77-8

temporality $35,39-40,47,49-50,71$, $92,105-9,112,118,122,127-30$, 159

tennis 43

tentacles 83-4

Tentacool 83

Tetris 100

texting see SMS

thermodynamics, laws of 114

thermostats 110

time see temporality

Tolkein, J.R.R. 5

Tomb Raider 30, 94

toys $14,15,17-9,22-6,27-9,39,40,43$, $57,61,63,64,68,70,72-3,76,78$, $79,88,89,90-1,113,115 \mathrm{n}, 123-4$, $126,133,138,140,144,147,156$, 158,160 n

Toy Story 144

traffic 15n, 158

transduction $9,17,26-8,30,31-2,57,64$, $71-2,77,88,98-9,118,144,151$, 158

transfer 27, 76, 144

transitional objects $115 \mathrm{n}$ transmedia systems 51, 67, 69, 70, 71-6, $77,79-85,88,89,118-19,138,156$

Treasure Island 134

turing test 105

Turkle, Sherry 100, 102

turn-based strategy games 106

Turner, Victor 41, 45, 50

turnips 107-8

tweens 38

unicorns 147

Valentine, Gill 51

vandalism 143

video cameras/recording $17,18,61,63$, 64

see also webcams

vikings $22-6$

violence, simulation of $12,78,138,140$, $142-3$

virtual, the $45-8,51,145$

virtual animals $12,45,53,73-4,91,97-8$, $105-9,115 \mathrm{n}$

virtual community $46-7$

virtual economy 98-9, 100, 105-9, 115n

virtual gravity see virtual physics

virtual physics $12,26,27,28-9,33 n, 64$, $66,90,92,126-7$

virtual reality $15,16 \mathrm{n}, 46-7,109,137$, 155

virtual space $10,14,18,26,28-9,32,46-8$, $52,64,112$

virtual worlds see virtual space

Wajcman, Judy 51, 58

Walkerdine, Valerie 39

Walther, Bo Kampfmann 43

wampas 120-1

Warhol, Andy 36

war toys, war games 140-2

Washington, George 122

Wasko, Janet 73-4

Watts, Jon 16n

Webb, Fr Damian 2

webcams 36, 40, 52

Weber, Sandra vi, 15, 20-1, 51, 72-3, 88, 154

Wegener-Spöhring, Gisela 143 
Weinbren, Grahame 111

Wells, H.G. 6

Who Wants to be a Millionaire? 153

Wiener, Norbert 94, 109

Willett, Rebekah 77-9, 140, 153

Williams, Raymond 38

Willis, Paul 55

Winnicott, D.W. 42, 115n, 127

winning states $9,14,43,134$

WipEout 110

Wise, J. Macgregor 103

witches 21, 147

World of Warcraft 91, 109, 115n, 159
Xbox 51, 143

Xbox Live 52, 59, 159

$X$-Men 145

Yoda 96-7, 122

youth $53 n, 55$

YouTube 40, 160

Zimmerman, Eric 109-10, 152

zoetropes 90

zombies 21, 147, 151, 161n

Zooks see Bamzooki

Zoo Tycoon 97-9, 100 